PFC/RR-90-8

\title{
A Method For Estimating Tokamak Poloidal Field Coil \\ Currents Which Incorporates Engineering Constraints
}

Stewart, William A.

May 1990

\author{
Plasma Fusion Center \\ Massachusetts Institute of Technology \\ Cambridge, Massachusetts 02139 USA
}

This work was supported by the author and his family. Reproduction, translation, publication, use and disposal, in whole or in part, by or for the United States government is permitted.

\section{DISCLAIMISR}

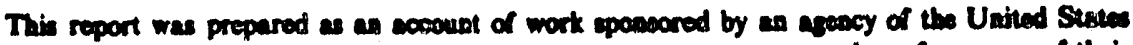

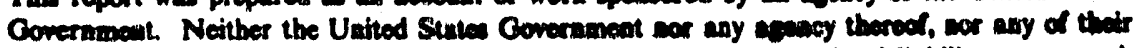

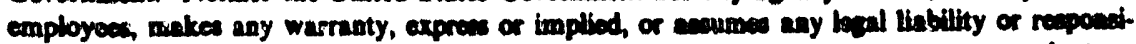

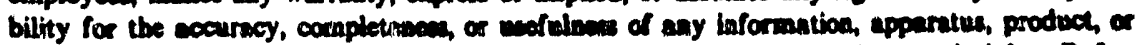

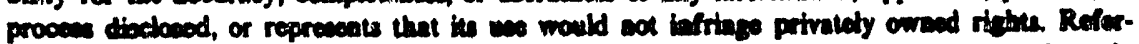

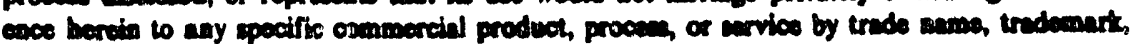

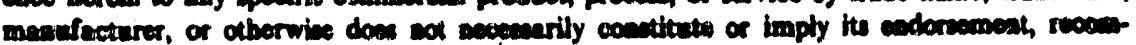

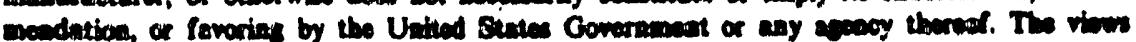

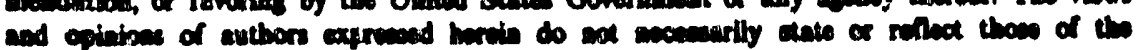

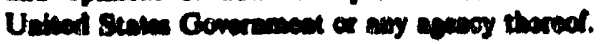




\title{
A Method For Estimating Tokamak Poloidal Field Coil Currents Which Incorporates Engineering Constraints
}

by

\author{
William Andrew Stewart \\ B.S., Carnegie-Mellon University (1985) \\ Submitted to the Department of Nuclear Engineering \\ in partial fulfillment of the requirements for the degrees of \\ Nuclear Engineer \\ and \\ Master of Science in Nuclear Engineering \\ at the \\ MASSACHUSETTS INSTITUTE, OF TECHNOLOGY \\ June 1990 \\ (C) William A. Stewart, 1990
}

The author hereby grants to MIT permission to reproduce and to distribute copies of this thesis document in whole or in part.

Signature of Author

Department of Nuclear Engineering May 15, 1990

Certified by

Jeffrey P. Freidberg

Professor, Departmant of Nuclear Engineering

Thesis Supervisor

Accepted by

Allan F. Henry

Chairman, Departmental Committee on Graduate Students 


\title{
A Method For Estimating Tokamak Poloidal Field Coil \\ Currents Which Incorporates Engineering Constraints
}

\author{
by \\ William Andrew Stewart \\ Submitted to the Department of Nuclear Engineering \\ on May 15, 1990, in partial fulfillment of the \\ requirements for the degrees of \\ Nuclear Engineer \\ and \\ Master of Science in Nuclear Engineering
}

\begin{abstract}
This thesis describes the development of a design tool for the poloidal field magnet system of a tokamak. Specifically, an existing program for determining the poloidal field coil currents has been modified to: (1) support the general case of asymmetric equilibria and coil sets, (2) determine the coil currents subject to constraints on the maximum values of those currents, and ( 3 ) determine the coil currents subject to limits on the forces those coils may carry. The equations representing the current limits and coil force limits are derived and an algorithm based on Newton's method is developed to determine a set of coil currents which satisfies those limits. Th resulting program allows the designer to quickly determine whether or not a given coil set is capable of supporting a given equilibrium.
\end{abstract}

Thesis Supervisor: Jeffrey P. Freidberg

Title: Professor, Department of Nuclear Engineering

Thesis Reader: S. Pekka Hakkarainen

Title: Researc' Scientist 


\section{Acknowledgments}

I would like to thank the many people who have helped me complete my education at MIT.

First, I would like to thank my thesis supervisor, Professor Jeffrey P. Freidberg, who originally proposed this project and allowed me to pursue it to my own satisfaction. Through his classes and our conversations, he has always challenged and inspired me, and I am indebted to him for his advice and support throughout my career at MIT.

I would also like to thank Dr. Scott Haney and Dr. S. Pekka Hakkarainen. Dr. Haney's thesis research provided the foundation upon which this thesis is built, and his help in clarifying many of the subtle points in his derivations has proven immeasurable. I would also like to thank him for his impeccable programming style, which greatly eased the amount of work I had to perform to decipher his programs. Dr. Hakkarainen gave me day-to-day support during the period while I was developing the primary algorithm used in this thesis. Additionally, his own work on the minimization problem discussed here clarified many of the issues involved in solving this class of problems.

Next, I would like to thank my friends at the MIT Plasma Fusion Center for the good times we have shared both at and away from work. These include: Dr. Martin Cireenwald, Sean Barnet, Justin Schwartz, Hanos Chaniotakis, Scot, Peng, Warren Krueger, Bob Birri and Tom and Sue Hsu. In their own way, each of these people has made my experience here quite enjoyable and $\mathrm{fun}$.

Finally, I would like to thank my parents, my brothers, my sister-in-law, my grandparents and all of my far-flung relatives for their concern and good wishes during my stay here. Additionally, I would like to thank my friends Ken and Alysa Studley, Tom and Anna Kovalcik, and Glemn Jo,pee.

This work is dedicated to my beloved fiancé, Donna Nichols, for her patience, love, support and incredible understanding throughout my eduction. 


\section{Contents}

1 Introduction $\quad 15$

1.1 Some Basic Plasma Physics . . . . . . . . . . . . . . . . . . . 16

1.2 The Poloidal Field System . . . . . . . . . . . . . . . . . . . . . . . . 19

1.3 Estimating PF Coil Currents . . . . . . . . . . . . . . . . . . 20

1.4 Extension to Asymmetric Systems f . . . . . . . . . . . . . . 21

1.5 Current Constraints . . . . . . . . . . . . . . . . 21

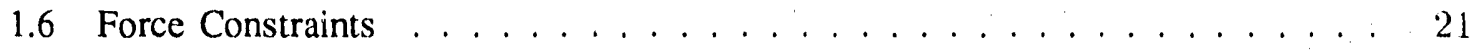

2 Review of Coil Current Solver $\quad 23$

2.1 Introduction . . . . . . . . . . . . . . . . . . . 23

2.2 Problem Statement . . . . . . . . . . . . . . . . . 21

2.3 Calculation of the Vacuum Field . . . . . . . . . . . . . . 28

2.3.1 Simplification of Grcen's Theorem . . . . . . . . . . . . . . . 29

2.3.2 Conductor Grouping . . . . . . . . . . . . . . . . . . . . . 30

2.3 .3 Fourier Analysis . . . . . . . . . . . . . . . . . . 31

2.4 Solution for Group Currents . . . . . . . . . . . . . . . . . . . . . . . . 33

2.5 summary . . . . . . . . . . . . . . . . . . . . . 34

$3 \quad$ Asymmetric Systems $\quad 37$

3.1 Discussion . . . . . . . . . . . . . . . . . . . 37

3.2 Generalization . . . . . . . . . . . . . . . . . . . . 39

3.3 verification . . . . . . . . . . . . . . . . . 41

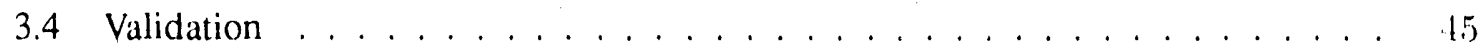


4 linear Constraints $\quad 55$

4.1 The Source of the Constraints . . . . . . . . . . . . . . . . 55

4.1 .1 Resistive Magnets . . . . . . . . . . . . . . . . . 55

4.1 .2 Superconducting Magnets . . . . . . . . . . . . . . 57

4.2 Problem Statement . . . . . . . . . . . . . . . . . . . . . 57

4.3 Finding a Feasible Point $\ldots \ldots \ldots \ldots \ldots$

4.4 Numerical Implementation $\ldots \ldots \ldots \ldots$

4.5 Avoiding Singularities . . . . . . . . . . . . . . . . . 62

4.6 Verification, Validation, and Testing . . . . . . . . . . . . . 63

4.7 Discussion . . . . . . . . . . . . . . . . . . . . . . . 69

5 Forces on PF Coils $\quad 73$

5.1 The Vector Potential . . . . . . . . . . . . . . . . . . . T3

5.1 .1 Vector Potential of a Distributed Current . . . . . . . . . 74

5.1 .2 Filamentary Currents . . . . . . . . . . . . . . $\tau_{4}$

5.2 Magnetic Energy of a System of Currents . . . . . . . . . . 75

5.2.1 Two Distributed Currents . . . . . . . . . . . . . . . 75

5.2 .2 A Single Distributed Current . . . . . . . . . . . . . 76

5.2 .3 Two Filamentary Currents . . . . . . . . . . . . . . 76

5.2 .4 A Single Filamentary Current . . . . . . . . . . . . . . . . 76

5.3 Force Computations . . . . . . . . . . . . . . . 77

5.3.1 Force Between Two Distributed Currents . . . . . . . . . . . . 78

5.3.2 Self Force of a Distributed Current . . . . . . . . . . . . . . . 79

5.3.3 Force Between Two Filamentary Currents . . . . . . . . . . . .

5.3.4 Self Force of a Filamentary Current . . . . . . . . . . . . . . 79

5.4 Modelling the Coil-Coil Forces . . . . . . . . . . . . . . . . 80)

5.5 Plasma-Coil Interaction Forces $\ldots \ldots \ldots \ldots 6$

5.5 .1 The Scalar Potential . . . . . . . . . . . . . . . . . .. .87

5.5.2 Gradient of the Scalar Potential . . . . . . . . . . . . . . . 88

5.6 Plasma Force Equations . . . . . . . . . . . . . . . . . . . . . . . . . . 90

6 Nonlinear Constraints $\quad 91$

6.1 The Source of the Constraints . . . . . . . . . . . . . . . . . 91 


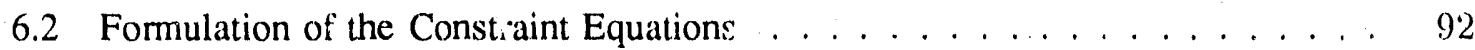

6.3 Verification, Validation, and Testing . . . . . . . . . . . . . . . 95

6.4 Constraining Grouped Currents . . . . . . . . . . . . . . . 99

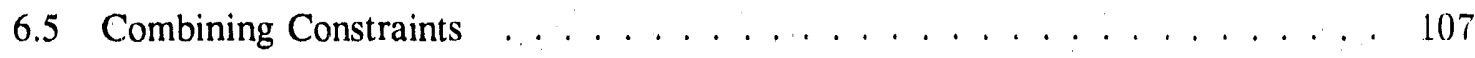

7 Conclusions and Recommendations $\quad 1.11$

A Verification of Asymmetric Program 113

A.1 Case 1: A Conventional Tokamak . . . . . . . . . . . . . . . . . . . 113

A.2 Case 2: An Elongated Tokamak . . . . . . . . . . . . . . . . 117

B Alcator C-Mod Coil Set $\quad 121$

C Symmetry Relations. $\quad 131$

C.1 Symmetry of $G\left(r, z ; r^{\prime}, z^{\prime}\right) \ldots \ldots \ldots \ldots \ldots \ldots$

C.1.1 Exchange of $r$ and $r^{\prime} \ldots \ldots \ldots \ldots \ldots \ldots \ldots$

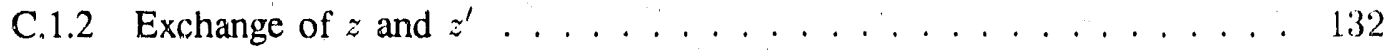

C.1.3 Results . . . . . . . . . . . . . . . . . . 133

C.2 Symmetry of $U_{j \rightarrow i} \ldots \ldots \ldots \ldots \ldots \ldots \ldots$

C.3 Symmetry of $M_{i j} \ldots \ldots \ldots \ldots \ldots \ldots$

D Derivation of the Intercoil Force Expressions $\quad 135$

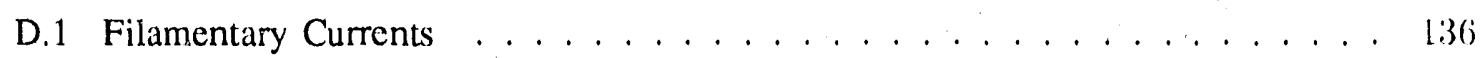

D.1.1 Force Between Two Coils . . . . . . . . . . . . . . . . . 136

D.1.2 Self Force of a Filamentary Current . . . . . . . . . . . . . 138

D.2 Distributed Currents . . . . . . . . . . . . . . . . . . . . . . . . 1399

D.2.1 Force Between Two Coils . . . . . . . . . . . . . . . . . . . . 139)

D.2.2 Self Force of a Distributed Current . . . . . . . . . . . . . . 1410

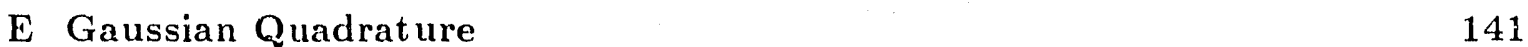




\section{List of Figures}

1-1 Geometry of a simple screw pinch. . . . . . . . . . . . . . 17

1-2 Geometry and coils of an ohmically heated tokamak. . . . . . . . . 19

2-1 Geometry of the plasma surface and conductors for use in the poloidal field coil current calculation . . . . . . . . . . . . . . . . . . . 25

3-1 Example of a symmetric plasma and coil set . . . . . . . . . . 38

3-2 Tangential poloidal field for the equilibrium presented in Figure $3-1 \ldots 38$

3-3 Example of a single null diverted plasma . . . . . . . . . . . . . . . 40

3-4 Tangential poloidal field of the single null plasma shown in Figure 3-3 . . . . 4 40

3-5 Plasma and coil set for the proposed Versator upgrade . . . . . . . . . . . . . 12

3-6 Poloidal fields for the Versator test case . . . . . . . . . . . . . . . . . . .4.

3-7 Symmetric filament model of plasma current . . . . . . . . . . . . . . . t6

3-8 Poloidal fields for symmetric test case . . . . . . . . . . . . . . . 48

$3-9$ Mux surface for the asymmetric case . . . . . . . . . . . . . . . . . 50

3-10 Poloidal field for the asymmetric test case . . . . . . . . . . . . . . 52

3-11 Alcator C-Mod single null test case . . . . . . . . . . . . . . . . 52

3-12 Poloidal fields of the Alcator C-Mod single null case f . . . . . . . . . 5.3

4-1 Alcator C-Mod equilibrium and coil set . . . . . . . . . . . . . . 6 (64t

4-2 Poloidal ficlds for the unconstrainer case . . . . . . . . . . . . . 65

4-3 Foloidal fields for one active constraint . . . . . . . . . . . . . . . 66

4.4 Poloidal fields for the case of two active constraints . . . . . . . . . . . . 6i7

4-5 Poloidal fields for two active constraints and one inactive constraint . . . . . . 6 (j8

4-6 Poloidal fields for the case of two active and two inactive constraints . . . . . . 69 
$5-1$ Simple coil model for computing the magnetic energy . . . . . . . . . . 75

5-2 Radial components of the force coefficient for an axially displaced coil . . . . . 81

5-3 Axial components of the force coefficient for an axially displaced coil . . . . . 81

5-4 Relative difference of the radial force coefficients for an axially displaced coil . $\quad 82$

5-5 Relative difference of the axial force coefficients for an axially displaced coil . $\quad 82$

5-6 Radial components of the force coefficient for a coil of varying radius . . . . . 83

5-7 Axial components of the force coefficient for a coil of varying radius . . . . . . 84

5-8 Radial component of the relative difference for a coil with a varying radius . . . 84

5-9 Axial component of the relative difference for a coil with a varying radius . . . 85

6-1 Alcator C-Mod coil set and equilibriurn . . . . . . . . . . . . . . . . 96

$6-2$ Poloidal fields for the unconstrained case . . . . . . . . . . . . . . 97

$6-3$ Poloidal fields for one force constraint $\ldots \ldots \ldots \ldots$

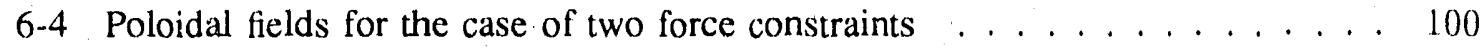

6-5 Poloidal fields for the case of three force constraints . . . . . . . . . . 101

6-6 Poloidal fields for the case of four force constraints. . . . . . . . . . . . 102

6-7 Poloidal fields for a force constraint on a set of grouped coils . . . . . . . . . . 104

6-8 Poloidal fields for a constrained set of grouped coils . . . . . . . . . . . 105

6-9 Poloidal fields for the case of a constrained set of grouped coils . . . . . . . . 106

6-10 Poloidal fields for the previous case with the grouping removed . . . . . . . 108

6-11 Poloidal fields for the general case of current and force constraints . . . . . . 109

A-1 A conventional tokamak coil set and circular plasma . . . . . . . . . . . 114

A-2 Poloidal fields for the conventional tokamak test case . . . . . . . . . . 116

A-3 Highly elongated tokamak plastna and coil set. . . . . . . . . . . . . I1.

A-4 Poloidal fields for the highly elongated tokamak test case f . . . . . . 119

E-1 Self-force coefficient as a function of the number of quadrature points used. . . 1.33 


\section{List of Tables}

3.1 Equilibrium parameters used for the Versator equilibrium. . . . . . . . . . . 42

3.2 Groupeù coil results for the Versator test case . . . . . . . . . . . . . . . . 43

3.3 Ungrouped coil results for the Versator case . . . . . . . . . . . . . . 43

3.4 Input plasma filament currents for the symmetric test case . . . . . . . . . 46

3.5 Input poloidal field coil currents for cPFC validation . . . . . . . . . . . . . 47

3.6 Symmetric test case coil current comparison . . . . . . . . . . . . . . . . . . 49

3.7 Input plasma filament currents for the asymmetric test case $\ldots \ldots \ldots \ldots$

3.8 Asymmetric test case coil current comparison . . . . . . . . . . . . . 51

3.9 Equilibrium parameters for the Alcator single null equilibrium. . . . . . . . . 53

4.1 Equilibrium data for the Alcator C-Mod test case. . . . . . . . . . . 6.4

4.2 Summary of the test results for the linear constraint algorithm . . . . . . . . T0

6.1 Equilibrium data for the Alcator C-Mod test casc. . . . . . . . . . . . . 96

6.2 Unconstrained coil forces and currents. . . . . . . . . . . . . . . . . 97

6.3 Coil forces and currents for one force constraint $\ldots \ldots \ldots$. . . . . . . 98

6.4 Coil forces and currents for the case of two force constraints. . . . . . . . . . 100

6.5 Coil forces and currents for the case of three force constraints. . . . . . . . 101

6.6 Coil forces and currents for the case of four force constraints. . . . . . . . . 102

6.7 Coil forces and currents for grouped coils subject to a force constraint. . . . . . 10.4

6.8 Coil forces and currents for grouped coils subject to force constraints. . . . . . 10.5

6.9 Coil forces and currents for the case of constrained grouped coils. . . . . . . . 106

6.10 Coil forces and currents for the previous case with ungrouped coils. . . . . . . 108

6.11 Coil forces and currents for the general case of current and force constraints. . . 109 
A.1 Equilibrium data for the conventional tokamak test case. . . . . . . . . . . . . 114

A.2 Grouped coil results for the conventional tokamak . . . . . . . . . . . 115

A.3 Ungrouped coil results for the conventional tokamak case . . . . . . . . . 115

A.4 Equilibrium data for the highly elongated tokamak. . . . . . . . . . . . . 118

A.5 Grouped coil results for the highly elongated tokamak . . . . . . . . . . . . 118

A.6 Ungrouped coil results for the highly elongated tokamak . . . . . . . . . . . . . 119

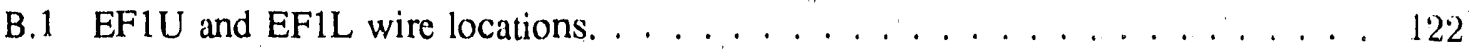

B.2 EF2U and EF2L wire locations. . . . . . . . . . . . . . . . 123

B.3 EF3U and EF3L wire locations. . . . . . . . . . . . . . . . . . . . . . 124

B.4 EFCU and EFCL wire locations. . . . . . . . . . . . . . 125

B.5 EF4U and EF4L wire locations. . . . . . . . . . . . . . . 126

B.6 $\mathrm{OH} 2 \mathrm{U}$ and $\mathrm{OH} 2 \mathrm{~L}$ wire locations. . . . . . . . . . . . . . 127

B.7 Wire locations of the $\mathrm{OH} 1 \mathrm{ohmic}$ transformer. . . . . . . . . . . . 128

B.7 Continued . . . . . . . . . . . . . . . . . . . . . . . . . . 129

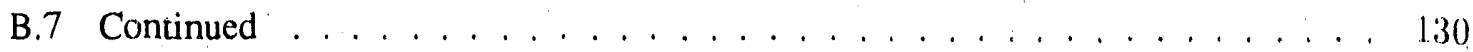




\section{Chapter 1}

\section{Introduction}

This thesis is concerned with the development of a design tool for the poloidal field magnet system of a tokamak. Currently, the design process for these systems proceeds along the following lines:

1. The desired plasma physics parameters are determined.

2. From these parameters, the poloidal field necessary to support the desired equilibrium is found.

3. The magnet positions are estimated from the required poloidal field.

4. Once the magnet positions have been determined, the rest of the engineering requirements (i.e. coil currents and forces) are found.

5. Steps 1 through 4 are iterated until the design is finalized.

Because of the complexity of a tokamak, the computer programs available to assist in the design process are quite complex themselves. This complexity makes these tools difficult to learn and use. Additionally, they are often quite slow, requiring anywhere from minutes to hours of processing time on a supercomputer. The result of all this is that a methodical scan over the range of physics parameters for a single design iteration is quite tedious. Furthermore, if a particular iteration is at an early phase in the design process, much of the detailed information produced by these programs may be unnecessary.

The slow execution times of the aforementioned prograrns arise from their general approach to the problem. They attempt to solve the equations describing the system as accurately and completely as possible. Haney [12] proposed that a tokamak designer might find a set of fast 
tools valuable for the first few iterations of the design process. The speed of these tools is achieved by sacrificing some of the accuracy of the solution, although in practice the differences between the fast and standard methods are only a few percent [15]. Once an advanced design is ready, it can be verified using the more accurate tools.

The design tool described herein is built on Haney's method for estimating the currents in the poloidal field magnets [13]. Here, his method will be extended to satisfy the following constraints:

- Realism, specifically the ability to model up-down asymmetric configurations

- The maximum current flowing in a coil

- The maximum force actiris on a coil

The realism constraint is more conceptual than physicul. The original implementation of Haney's method was applicable only to a limited subset of tokamak configurations. The removal of that limit is the subject of Chapter 3. Finding the coil currents necessary to support an equilibrium subject to limits on the maximum current flowing in those coils is the subject of Cnapter 4. To determine the coil currents such that the limits on the forces acting on the coils first requires finding the forces acting on those coils. The derivation of the expressions for the coil forces is presented in Chapter 5, and determining the coil currents such that the limits on the coil forces are satisfied is discussed in Chapter 6 . Further introduction to these topics is given later in this chapter. First though, it is necessary to briefly discuss the relevant plasma physics and describe the poloidal field system in detail.

\subsection{Some Basic Plasma Physics}

The plasma physics required to design a tokamak is contained in the theory of ideal magnetohydrodynamics (MHD). Solving these equations for a given set of plasma parameters fixes the boundary conditions required to solve these equations. These boundary conditions, in turn, make it possible to estimate the currents in the poloidal field magnets.

The MHD equations describing a static plasma equilibrium are [3]:

$$
\begin{aligned}
\mathbf{J} \times \mathbf{B} & =\nabla p \\
\nabla \times \mathbf{B} & =\mu_{0} \mathbf{J}
\end{aligned}
$$




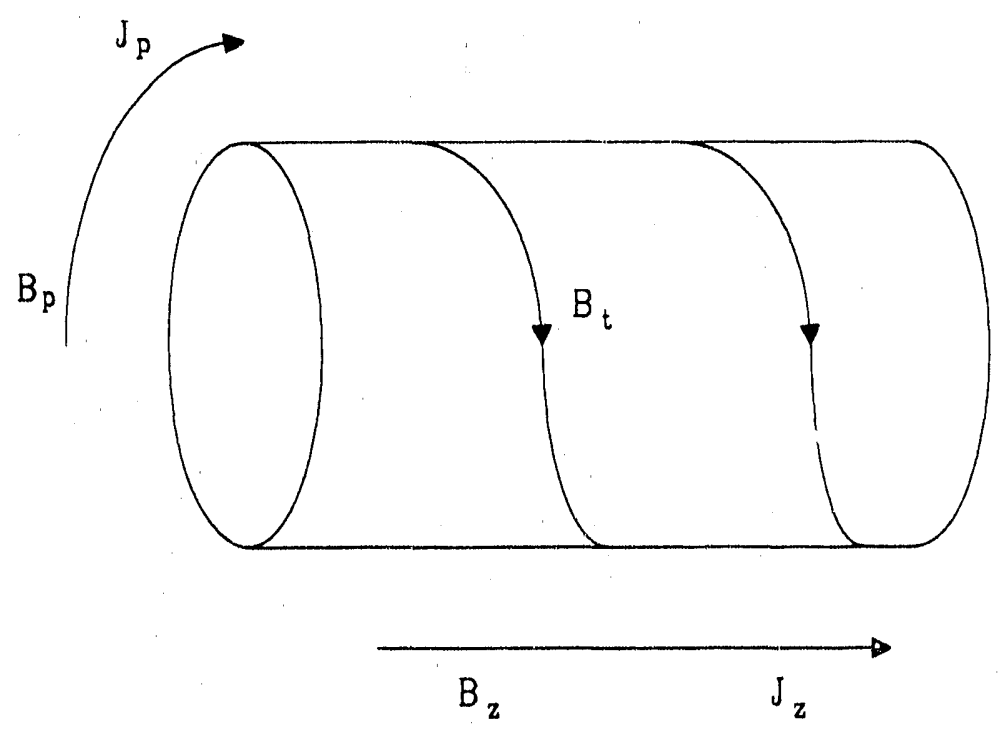

Figure 1-1: Cieometry of a simple screw pinch.

$$
\nabla \cdot \mathbf{B}=0
$$

Equations 1.2 and 1.3 are just Maxwell's equations. The equilibrium state itself is described by Equation 1.1, which states that the force due to the gradient of the plasma pressure is balanced by the magnetic force acting on the plasma.

The simplest general example of the force balance equation arises by considering a simple screw pinch. This system consists of a cylindrical plasma with both angular and axial components of $\mathbf{J}$ and $\mathbf{B}$, as shown in Figure 1-1. The resulting magnetic field vector is helical, similar to the threads on a screw. Allowing the field and current density 20 vary only in the radial direction ensures that Equation 1.3 is satisfied:

$$
\frac{1}{r} \frac{\partial B_{\theta}(r)}{\partial \theta}+\frac{\partial B_{z}(r)}{\partial z}=0
$$

Combining Equations 1.1 and 1.2 leads to the pressure balance equation for this system, namely:

$$
\frac{d}{d r}\left(p+\frac{B_{\theta}^{2}+B_{z}^{2}}{2 \mu_{0}}\right)+\frac{B_{\theta}^{2}}{\mu_{0} r}=0
$$


Examining Equation 1.5 shows that knowledge of the profiles of the plasma pressure and one of the magnetic field components makes it possible to determine the profile of the other magrietic field component. Assuming that the axial compronent of the field is known, the azimuthal component can be determined from

$$
r \frac{d}{d r}\left(\frac{B_{\theta}^{2}}{2 \mu_{0}}\right)+\frac{B_{\theta}^{2}}{\mu_{0}}=-r \frac{d p}{d r}-r \frac{d}{d r}\left(\frac{B_{z}^{2}}{2 \mu_{0}}\right)
$$

To find this component of the field exactly, though, requires knowledge of the boundary conditions.

In general, the plasma is supported in equilibrium by an extermally applied magnetic field. This field is generated by a set of current carrying conductors surrounding the plasma. Furthermore, a vacuum region is assumed to exist between the plasma and the conductors. From this, it is possible to define the plasma surface (which in general is non-circular) as the curve along which the plasma pressure is effectively zero.

The boundary conditions at the plasma surface are given by

$$
\begin{aligned}
\left.\hat{\mathbf{n}} \cdot \mathbf{B}\right|_{a} & =\left.\hat{\mathbf{n}} \cdot \hat{\mathbf{B}}\right|_{a} \\
\hat{\mathbf{n}} \times\left.\mathbf{B}\right|_{a} & =\hat{\mathbf{n}} \times\left.\hat{\mathbf{B}}\right|_{a} \\
\left.B^{2}\right|_{a} & =\left.\hat{B}^{2}\right|_{a}
\end{aligned}
$$

where $\mathrm{B}$ is the magnetic field within the plasma, $\hat{\mathrm{B}}$ is the vacuum magnetic field, and $\hat{\mathrm{n}}$ is the outward pointing unit vector normal to the plasma surface. These boundary conditions ensure that the normal and tangential components of the magnetic field and the magnetic pressure are continuous across the plasma surface and it is assumed that no surface currents flow. Additionally, the condition $\mathbf{B} \cdot \nabla p=0$ implies that $\left.\mathbf{n} \cdot \mathbf{B}\right|_{a}=0$, which simplifies Equation 1.7 to

$$
\left.\hat{\mathbf{n}} \cdot \hat{\mathbf{B}}\right|_{a}=0
$$

The vacuum field $\hat{\mathbf{B}}$ is determined from

$$
\hat{\mathrm{B}}=\mathrm{B}_{\mathrm{a}}+\tilde{\mathrm{B}}
$$

where $\mathrm{B}_{\mathrm{a}}$ is the rnagnetic field due to the conductors and $\tilde{\mathrm{B}}$ is the vacuum magnetic field of the plasma. 


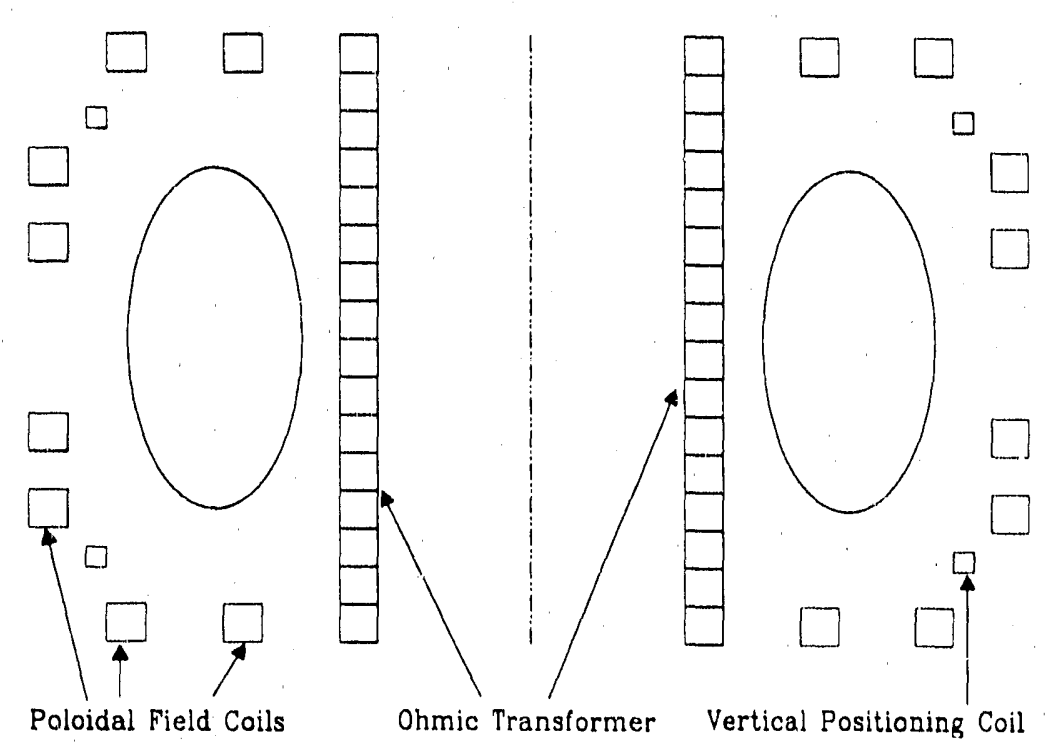

Figure 1-2: Geometry and coils of an ohmically heated tokamak.

Extending the MHD equations to the toroidally axisymmetric geometry of a tokamak results in the Grad-Shafranov equation, given by

$$
\Delta^{*} \psi=-\mu_{0} R^{2} \frac{d p}{d \psi}-F \frac{d F}{d \psi}
$$

where $\psi$ is a stream function for the poloidal magnetic field, $\Delta^{*}$ is an elliptic operator given by

$$
\Delta^{*}=R \frac{\partial}{\partial R}\left(\frac{1}{R} \frac{\partial}{\partial R}\right)+\frac{\partial^{2}}{\partial Z^{2}}
$$

$p$ is the plasma pressure, and $F$ is a function related to the net poloidal current. All of the equilibria presented in this thesis were produced by a system which generates an approximate solution to the Grad-Shafranov equation.

\subsection{The Poloidal Field System}

Figure 1-2 shows the general geometry of an ohmically heated tokamak and the major components of the poloidal field coil system. The first major component is the ohmic transformer. This set of 
coils acts as the primary winding of a large transformer, with the plasma acting as the secondary. The current in the plasma is induced by varying the current in the ohmic coils.

The second system is made up of the poloidal field coils themselves. These coils are responsible for shaping the plasma and providing the radial and toroidal pressure balance needed to maintain the plasma in equilibrium.

The third system is a set of feedback controlled vertical positioning coils. These coils are necessary to stabilize the vertical instability associated with highly elongated equilibria. As the plasma drifts in the vertical direction, current is applied to these coils in order to push the plasma back to the midplane of the machine.

\subsection{Estimating PF Coil Currents}

Equations 1.8 and 1.10 describe the boundary conditions which the equilibrium must satisfy. The equilibrium information provided by the Grad-Shafranov equation provides the poloidal magnetic field at the plasma surface. Combining this information makes it possible to determine the required currents in the external sls. Chapter 2 reviews a method for quickly estimating these currents. This method is based on an application of the scalar version of Green's theorem to compute the tangential component of the vacuum magnetic field at the surface of the plasma. This quantity is expressed in the form

$$
\hat{B}_{p}(\mu)=b^{(1)}(\mu)+\sum_{j=1}^{J} b_{j}^{(2)}(\mu) I_{J}
$$

where $\mu$ is a poloidal angular variable, $I_{j}$ is the unknown current in the $j$ th poloidal field coil, and the terms $b^{(1)}(\mu)$ and $b_{j}^{(2)}(\mu)$ are determined by requiring that $\mathbf{n} \cdot \hat{\mathbf{B}}=0$ on the plasma surface for any arbitrary set of $I_{j}$.

The currents are determined by minimizing a functional of the form

$$
\epsilon_{I}=\frac{\int_{S_{p}}\left(\hat{B}_{p}-B_{p}\right)^{2} d S}{\int_{S_{p}} B_{p}^{2} d S}
$$

where $B_{p}$ is the tangential component of the poloidal field just inside the plasma surface. Minimizing this functional form ensures that the discontinuity in the tangential component of the magnetic field is as small as possible.

It can be shown that the set of currents that minimize $t_{i}$ satisfy a simple set of linear equations. The currents can, therefore, be found without the need of a potentially fragile and 
time consuming iteration process. As a result, this procedure - implemented in a program called cPFC - is extremely fast and robust.

\subsection{Extension to Asymmetric Systems}

As implemented in the original version of the CPFC program, the method for estimating the coil currents described in the previous section was only applicable to up-down symmetric systems. These systems have both equilibria and poloidal field coil sets which are perfectly symmetric about the midplane of a tokamak. Requiring the system to be symmetric limited the applicability of the cPFC program.

Chapter 3 describes the changes made to CPFC to facilitate its use on asymmetric systems. Such systems include systenis with asymmetries in the coil set due to engineering considerations, asymmetric field structures due to the presence of vertical positioning coils, or asymmetric plasmas such as those found in single-null diverted tokamaks.

\subsection{Current Constraints}

Chapter 4 describes a method for estimating the poloidal field coil currents in light of constraints on those currents. These constraints can arise in several ways. First, the power supply generating the current in a given coil may simply limit the maximum current that coil can carry. Second, the current in a resistive coil may generate so much heat in the coil that the maximum temperature allowed in the coil is exceeded. If this maximum temperature corresponds to the melting point of the coil material, the coil could fail - a situation which should clearly be avoided if possible. Finally, if the coil is superconducting, the maximum current allowed in the coil is simply the critical current of the coil material.

\subsection{Force Constraints}

The poloidal field coils all experience a force proportional to $\mathrm{J} \times \mathrm{B}$, where $\mathrm{J}$ is the current derisity in the coil and $\mathrm{B}$ is the total magnetic field sampled by the coil. The coils and their support structure must be capable of withstanding these forces, or else they will fail. The equations needed to compute the forces on the coils are derived in Chapter 5. With these expressions for 
the forces, it is possible to apply the method developed in Chapter 4 for simple current constraints to the force constraint problem, which is the subject of Chapter 6 . 


\section{Chapter 2}

\section{Review of Coil Current Solver}

The basic foundation for the thesis is the analysis carried out by Haney [12]. In this chapter, his work on finding the poloidal field coil currents is reviewed and summarized. For purposes of clarity, some of the notation has been changed and elaborations have been made to some derivations wherever appropriate. It should also be noted that only information relevant to the current work has been included. For specific details the reader is referred to [12].

\subsection{Introduction}

Exact computation of the poloidal field coil currents requires the self-consistent solution of Maxwell's equations for the magnetic fields in the plasma and the vacuum regions of a tokamak. The plasma field can be obtained by solving the Grad-Shafranov equation, and the vacuum field can be computed from Laplace's equation. Boundary conditions imposed at the plasma surface connect the two solutions. The assumption that the plasma current and pressure vanish smoothly at the plasma surface dictates that the poloidal magnetic field be continuous across the plasma surface.

Generally, it is not possible to find a finite set of currents that satisfy the governing equations and the boundary conditions when both the plasma surface and the conductor locations are fixed. This is because a finite set of conductor currents is not capable of exactly satisfying the boundary conditions at an infinite number of points along the plasma surface. To compute the exact solution, the plasma surface is allowed to vary with the conductor currents. Since the plasma surface can move, this is referred to as the "free boundary" equilibrium problem.

The free boundary method makes it possible to determine the plasma and vacuum magnetic 
fields exactly and self-consistently. The disadvantage is that this method represents a difficult and time-consuming numerical computation. Another possible method involves the sacrifice of some accuracy by maintaining a fixed plasma surface. This assumption makes it impossible to match the boundary cr nditions exactly - there will be some jump in the magnetic field at the plasma surface. However, for any given set of conductors, there will in general be some set of currents which minimizes the jump in the field.

The reriainder of this chapter describes a method for determining the poloidal field coil currents in the above manner. Much of this procedure consists of the computation of the vacuum field at the plasma surface. This is accomplished by using Green's theorem to derive an integral equation for the scalar magnetic potential at the plasma surface. By Fourier analyzing this integral equation, an equivalent linear system is found which can be solved by standard techniques. The scalar magnetic potential can then be used to represent the vacuum magnetic field in terms of the unknown conductor currents. The conductor currents are then found by minimizing the root-mean-square jump in the field across the plasma surface.

\subsection{Problem Statement}

The first step in determining the conductor currents involves stating the problem in precise mathematical terms. Figure $2-1$ shows the surface of a toroidally axisymmetric plasma. The plasma surface is characterized by

$$
\begin{aligned}
& R=R_{p}(\mu) \\
& Z=Z_{p}(\mu)
\end{aligned}
$$

where $\mu$ is an arbitrary angular coordinate.

With this parameterization of the plasma surface, it is possible to define a number of quantities, including the unit normal vector,

$$
\mathbf{e}_{\mathbf{n}}=\frac{1}{Q}\left[Z_{\mu} \mathbf{e}_{\mathbf{R}}-R_{\mu} \mathbf{e}_{\mathbf{Z}}\right]
$$

and the unit tangent vector,

$$
\mathbf{e}_{\mathrm{t}}=\frac{1}{Q}\left\{R_{\mu} \mathbf{e}_{\mathrm{R}}+Z_{\mu} \mathbf{e}_{\mathrm{Z}}\right\}
$$

where $\mathbf{e}_{\mathrm{R}}$ and $\mathbf{e}_{\mathrm{Z}}$ are the unit vectors in the $\mathrm{R}$ and $\mathrm{Z}$ coordinate directions respectively, and

$$
Q \equiv\left[R_{\mu}^{2}+Z_{\mu}^{2}\right]^{1 / 2}
$$




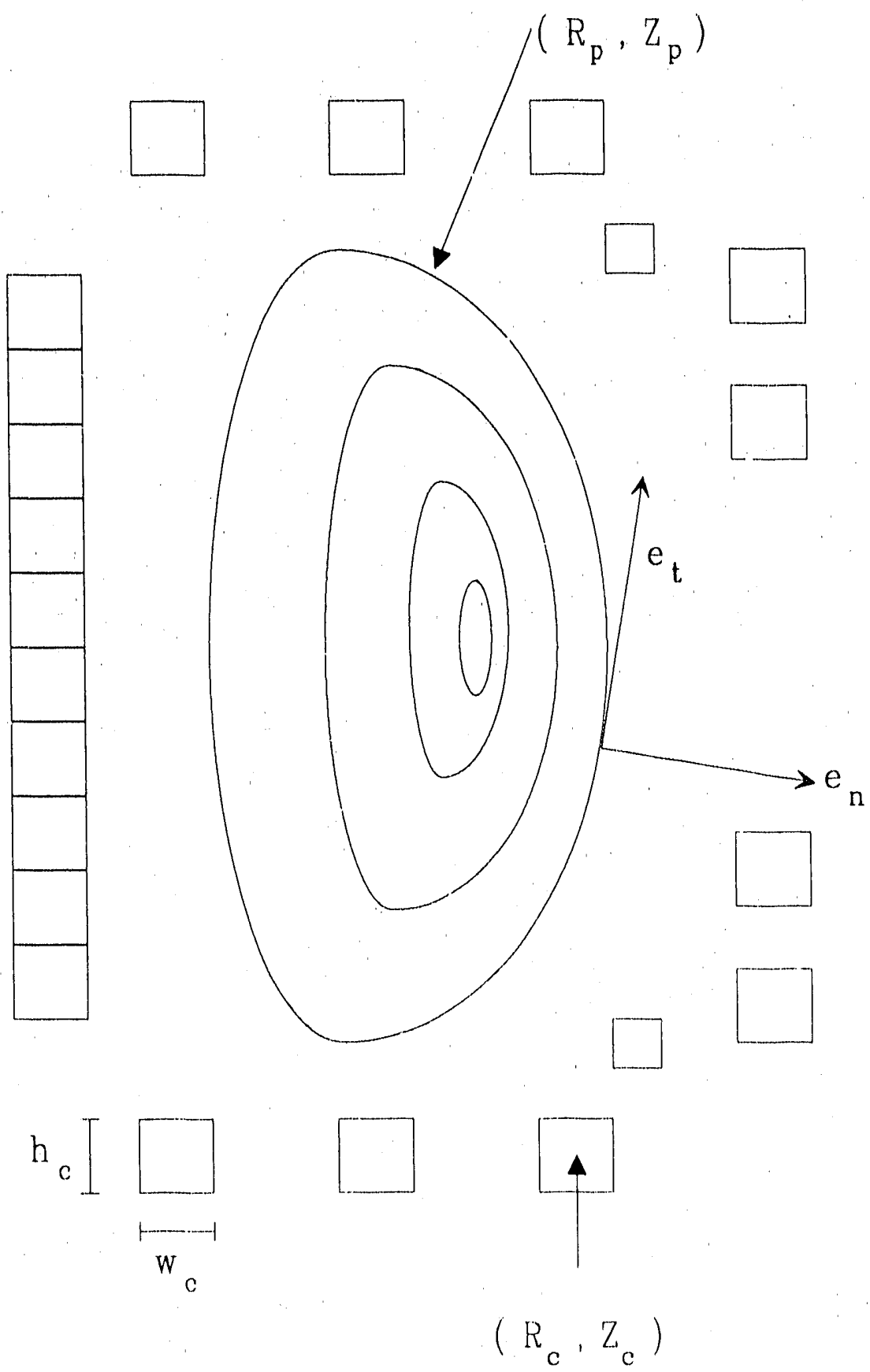

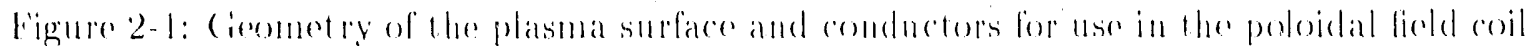
current ralculation 
From the definitions of the unit vectors, it is possible to define the normal a derivatives with respect to the plasma surface. The normal derivative is given by

$$
Q \mathbf{e}_{\mathbf{n}} \cdot \nabla \equiv \frac{\partial}{\partial n}=Z_{\mu} \frac{\partial}{\partial R}-R_{\mu} \frac{\partial}{\partial Z}
$$

and the tangential derivative by

$$
Q \mathbf{e}_{.} \cdot \nabla \equiv \frac{\partial}{\partial \mu}=R_{\mu} \frac{\partial}{\partial R}+Z_{\mu} \frac{\partial}{\partial Z}
$$

The differential element of arc length is computed from

$$
d s^{2}=d R_{p}^{2}+d Z_{p}^{2}=R_{\mu}^{2} d \mu+Z_{\mu}^{2} a^{i} \mu=Q^{2} d \mu^{2}
$$

and the differential surface area is

$$
d i S^{\prime}=R d s d \phi=R Q d \mu d \phi
$$

The plasma depicted in Figure 2-1 is assumed to be surrounded by vacuum. Within this vacuum region there are a total of $J$ toroidally axisymmetric conductors. The position of each conductor is given by $\left(R_{j}, Z_{j}\right)$. These conductors are treated as filaments to simplify the calculation of the coil currents, but in order to compute the resistance of the coil, each conductor is assumed to have a rectaneular cross-section with a width $w$, and height $h_{j}$. The goal of the aralysis, then, is to determine the current $I_{j}$ flowing in each conductor.

Calculation of the coil currents requires knowledge of the plasma and vacuum magnetic fields at the plasma surface. For the purpose of this derivation, the langential field at the plasma surface due to the plasma is assumed known from the solution to the Grad-Shafranov equation. The magnetic field in the vacuum region $\left(B_{v}\right)$ can then be written as the sum of the plasma field $\left(\mathrm{B}_{\mathrm{p}}\right)$ and the field due to the external coils $\left(\mathrm{B}_{\mathrm{c}}\right)$ :

$$
\mathrm{B}_{\mathrm{v}}=\mathrm{B}_{\mathrm{p}}+\mathrm{B}_{\mathrm{c}}
$$

Within the vacuum region, the field due to the plasma satisfies

$$
\begin{gathered}
\nabla \cdot \mathrm{B}_{\mathrm{p}}=0 \\
\Gamma \times \mathrm{B}_{\mathrm{p}}=0
\end{gathered}
$$

and is required to remain regular everywhere. It is then possible to express $B_{P}$, as

$$
B_{p}=\Gamma \phi+B_{i}
$$


where $\phi$ is the scalar maynetic potential and $\mathrm{B}_{\mathrm{i}}$ is the field due to a single filament assumed to carry all of the plasma current and located at the plasma magnetic axis $\left(R_{m}, Z_{m}\right)$. The exact location of this filament is not critical and does not affect the results. From equation 2.11 the scalar potential satisfies Laplace's equation

$$
\nabla^{2} \phi=0
$$

The filamentary plasma field $B_{i}$ has been introduced into this formulation to guarantec that $\phi$ is single-valued. This is necessary to ensure that the application of Ampere's law to the plasma boundary yields the correct value of the plasma current.

From magnetostatics, the $\mathrm{B}_{\mathrm{i}}$ ficld can be expressed as

$$
\mathrm{B}_{\mathrm{i}}=\mu_{0} I_{p} \frac{\nabla \Psi_{p} \times \mathbf{e}_{i}}{R}
$$

where

$$
\Psi_{p}=\frac{\left(R R_{m}\right)^{1 / 2}}{2 \pi}\left[\frac{\left(2-k_{p}^{2}\right) K\left(k_{p}\right)-2 E\left(k_{p}\right)}{k_{p}}\right]
$$

$K^{\prime}$ and $E$ are the complete elliptic integrals of the first and second kind, respectively, and

$$
k_{p}^{2}=\frac{4 R R_{m}}{\left(R+R_{m}\right)^{2}+\left(Z-Z_{m}\right)^{2}}
$$

The $J$ conductors are assumed to be small in cross-section and far from the plasma surface (relative to the dimensions of the conductor). This makes it possible to compute the applied field $\mathrm{B}_{\mathrm{C}}$ in a manner similar to $\mathrm{B}_{\mathrm{i}}$ :

$$
\mathbf{B}_{\mathbf{c}}=\sum_{j=1}^{J} \mu_{0} I_{j} \frac{\Gamma \Psi_{j} \times \mathbf{e}_{i}}{R}
$$

where

$$
\Psi_{J}=\frac{\left(R R_{j}\right)^{1 / 2}}{2 \pi}\left[\frac{\left(2-k_{j}^{2}\right) h\left(k_{j}\right)-2 K^{\prime}\left(k_{j}\right)}{k_{j}}\right]
$$

and

$$
h_{1}^{2}=\frac{1 R R_{1}}{\left(R+R_{1}\right)^{2}+\left(Z-Z_{1}\right)^{2}}
$$

One property of the ideal MHD model is that the plasma is perfectly conducting. This requires that the component of the magnetic field normal to the plasma surface vanish within and just outside of the plasma surface:

$$
\left.\mathbf{e}_{11} \cdot \mathrm{B}_{\mathrm{i}}\right|_{s_{11}}=\left.\mathbf{e}_{11} \cdot \mathrm{B}_{\mathrm{v}}\right|_{s_{1}}=0
$$


where $B_{i}$ is the plasma field inside the plasma. The solution of the Grad-Shafranov equation for the plasma equilibrium already satisfy this condition. For the vacuum field, however, it is necessary to apply Equation 2.21 explicitly. In the following section, it is shown that this condition allows the tangential component of the vacuum ficld to be expressed in the form

$$
B_{v}(\mu)=b^{(1)}(\mu)+\mathbf{b}^{(2)}(\mu) \cdot \mathbf{i}
$$

where $\mathrm{i}$ and $\mathrm{b}^{(2)}$ are column vectors of length $J$ containing the currents $I_{j}$ and a set of geometric coefficients $b_{j}^{(2)}$ respectively. The currents $I_{j}$ are then determined by minimizing the functional

$$
\epsilon_{I}(\mathrm{i})=\frac{\int_{S_{p}}\left(B_{p}-B_{u}\right)^{2} d S^{\prime}}{\int_{S_{p}} B_{p}^{2} d S^{\prime}}
$$

where $B_{p}$ and $B_{v}$ represent the tangential components of the plasma and vacuum ficlds, respectively. Equation 2.23 can be thought of as the net deviation from exact radial pressure balance. Minimization of $\epsilon_{l}$ is thus equivalent to minimizing the net deviation from exact radial pressure balance at the plasma boundary.

\subsection{Calculation of the Vacuum Field}

The method used to determine the vacuum field $\mathrm{B}_{\mathrm{v}}$ is based on an application of Green's theorem [20]. This choice is facilitated by the fact that the ficld must only be calculated on the plasma surface. Additionally, several analyses have shown that this formalism is well suited for fast numerical computation $[7,5,6,11]$.

Green's theorem for the scalar magnetic potential is written as

$$
\sigma \phi(\mathbf{r})+\int_{S_{p}}\left[\phi\left(\mathbf{r}^{\prime}\right)\left(\mathbf{e}_{11}^{\prime} \cdot \Gamma^{\prime} \hat{i^{\prime}}\left(\mathbf{r}, \mathbf{r}^{\prime}\right)\right)-\left(i\left(\mathbf{r}, \mathbf{r}^{\prime}\right)\left(\mathbf{e}_{11}^{\prime} \cdot \Gamma^{\prime} \phi\left(\mathbf{r}^{\prime}\right)\right)\right] d S^{\prime}=0\right.
$$

In this equation, $\mathbf{r}^{\prime} \equiv\left(R^{\prime}, \varphi^{\prime}, Z^{\prime}\right)$ and $\mathbf{r} \equiv(R, \varphi, \%)$. Additionally, $\mathbf{r}$ denotes the observation point and $\mathbf{r}^{\prime}$ represents the variables of integration. Also, a regularity condition is imposed on so that the integral over an infinite bounding surface $\left(S^{\prime}\right)$ vanishes.

As discussed carlier, the plasma surface coordinates are parameterized with respect to $\mu$, so that $R^{\prime}=R_{p}\left(\mu^{\prime}\right)$ and $Z^{\prime}=Z_{p}\left(\mu^{\prime}\right)$. The function $C_{i}$ is the infinite space Green's function given by

$$
\hat{H}\left(\mathbf{r} \cdot \mathbf{r}^{\prime}\right)=-\frac{1}{|\pi| \mathbf{r}^{\prime}-\mathbf{r} \mid}
$$


where

$$
\left|\mathbf{r}^{\prime}-\mathbf{r}\right|=\left[R^{2}+R^{\prime 2}-2 R R^{\prime} \cos \left(\varphi^{\prime}-\varphi\right)+\left(Z^{\prime}-Z\right)^{2}\right]^{1 / 2}
$$

Finally, $\sigma$ is a coefficient which depends on the location of the observation point relative to the plasma surface, and is given by

$$
\sigma= \begin{cases}1 & \text { if } \mathbf{r} \text { is outside the plasma } \\ \frac{1}{2} & \text { if } \mathbf{r} \text { is on the plasma surface } \\ 0 & \text { if } \mathbf{r} \text { is inside the plasma. }\end{cases}
$$

Since the goal of this analysis is to compute $\phi$ on the plasma surface, $O$ is taken to be $1 / 2$, and $R$ and $Z$ are set to $R_{p}(\mu)$ and $Z_{p}(\mu)$, respectively.

\subsubsection{Simplification of Green's Theorem}

Through the assumption of toroidal axisymmetry, it is possible to climinate the $\varphi$ dependence in the integral of equation 2.24. This reduces equation 2.24 to

$$
\frac{1}{2} \phi(\mu)+\int_{0}^{2 \pi}\left[\phi\left(\mu^{\prime}\right) \frac{\partial\left(c^{\prime}\left(\mu, \mu^{\prime}\right)\right.}{\partial n^{\prime}}-\left(i\left(\mu, \mu^{\prime}\right) \frac{\partial \phi(\phi)}{\left.\partial n^{\prime}\right)}\right] R^{\prime} d \mu^{\prime}=0\right.
$$

where $G$ is the reduced Green's function defined as

$$
\left(i=\int_{0}^{2 \pi} \operatorname{li} d \varphi^{\prime}\right.
$$

Integrating equation 2.29 gives

$$
C_{i}=-\frac{k \cdot k(k)}{2 \pi \sqrt{k R^{\prime}}}
$$

where $k_{i}$ is given by

$$
k^{2}=\frac{4 R R^{\prime}}{\left(R^{\prime}+R\right)^{2}+\left(Z^{\prime}-Z\right)^{2}}
$$

The solution of equation 2.28 requires the evaluation of the normal derivatives of $(i$ and $d \%$. Using the definition of the normal derivative (equation 2.6) and the identities [10]

$$
\begin{aligned}
& \frac{d E\left(k_{i}\right)}{d k_{i}}=\frac{1}{k_{i}}\left[k_{i}\left(k_{i}\right)-k\left(k_{i}\right)\right] \\
& \frac{d N\left(k_{i}\right)}{d k_{i}}=\frac{1}{k_{i}}\left[\frac{E\left(k_{i}\right)}{1-k^{2}}-h\left(k_{i}\right)\right]
\end{aligned}
$$

results in

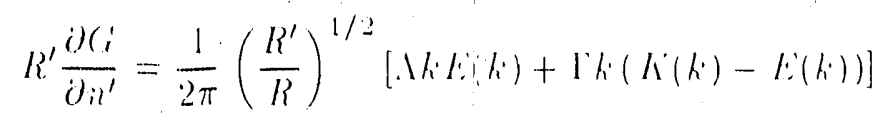


where

$$
\begin{aligned}
& \Lambda=\frac{Z_{\mu}^{\prime}\left(R^{\prime}-R\right)-R_{\mu}^{\prime}\left(Z^{\prime}-Z\right)}{\left(R^{\prime}-R\right)^{2}+\left(Z^{\prime}-Z\right)^{2}} \\
& \Gamma=\frac{Z_{\mu}^{\prime}}{2 R^{\prime}}
\end{aligned}
$$

The normal derivative of $\phi$ can be found by invoking the boundary condition on $\mathrm{B}_{\mathrm{v}}$, namely

$$
R^{\prime} \frac{\partial \phi}{\partial n^{\prime}}=\frac{\partial}{\partial \mu^{\prime}}\left(\mu_{0} I_{p} \Psi_{p}+\sum_{j=1}^{J} \mu_{0} I_{j} \Psi_{j}\right)
$$

Since no assumptions have been made about either the plasma shape or the location of the various conductors, equation 2.37 shows that it is always possible to make the normal component of the vacuum field exactly vanish at the plasma surface.

\subsubsection{Conductor Grouping}

For a given PF system, it often occurs that several conductors are constrained to carry the same current, while others carry a fixed current dictated by external conditions. Thus, the number of unique currents that must be determined is often much less than $\%$. Incorporating these cases now may save a significant amount of computation.

Generally, conductors can be constrained to carry the same current for a number of reasons. One such reason is conductor geometry. In general, several filaments are used to model the windings of a finite sized coil. These conductors are linked in series electrically, causing them to carry the same current. Another reason is symmetry. Often, the conductors are located symmetrically with respect to the midplane of the machine $(Z=0)$. This case usually occurs for up-down symmetric plasmas. Finally, it may be the case that there are too few power supplies to drive each coil independently, so some coils must be grouped together.

Fixed conductor currents can also occur for a number reasons. One exumple of this is the flat-top portion of an shmically heated discharge. In this phase of operation, the curreni in the ohmic transformer is preprogrammed to generate a constant plasma current. Calculation of the poloidal field system currents at a given time requires taking a "snapshot" of the equilibrium and computing the coil currents while the ohmic stack current is held constant at its preprogrammed value.

Accounting for grouped and fixed currents is straightforward. It is assumed that the currents in $F$ of the conductors are fixed. The remaining conductors are assumed to belong to one of $(i$ 
groups, where each conductor in each group is con:trained to carry the same current $I_{g}$. The $C i$ currents are then computed using the technique described in the next section.

From the above discussion, the vacuum field due to the conductors $\left(B_{C}\right)$ can be expressed as

$$
\mathbf{B}_{\mathbf{c}}=\mu_{0} I_{p} \frac{\nabla \Psi_{F} \times \mathbf{e}_{\phi}}{R}+\sum_{g=1}^{c} \mu_{0} I_{g} \frac{\nabla \Psi_{g} \times \mathbf{e}_{\phi}}{R}
$$

where

$$
\Psi_{F}=\sum_{j=1}^{J} \frac{I_{j}}{I_{p}} \delta_{j}^{(F)} \Psi_{l}
$$

and

$$
\Psi_{g}=\sum_{j=1}^{J} \delta_{j}^{(g)} \Psi_{j}
$$

Here, $\Psi_{F}$ accounts for the poloidal flux from the conductors carrying fixed currents, while $\Psi_{3}$ represents the total poloidal flux from the $g$ th conductor group. The selection functions $\delta_{j}(F)$ and $y_{j}^{(, g)}$ are defined by

$$
\begin{aligned}
& \delta_{j}^{(F)}= \begin{cases}1 & \text { if } I_{j} \text { is a fixed current, } \\
0 & \text { otherwise. }\end{cases} \\
& \delta_{j}^{(y)}= \begin{cases}1 & \text { if conductor } j \text { is in group } y, \\
0 & \text { otherwise. }\end{cases}
\end{aligned}
$$

Utilizing these results to rewrite equation 2.37 gives

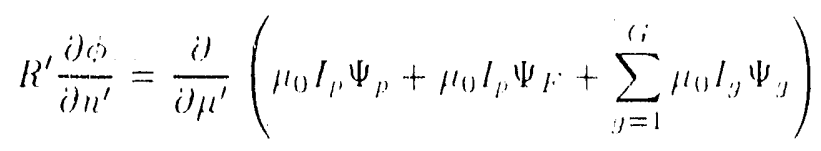

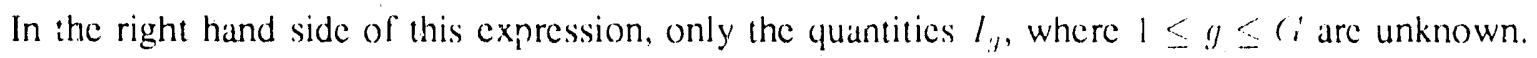

\subsubsection{Fourier Analysis}

Equation 2.28 can be solved through standard Fourier analysis techniques. This is done by first expanding and its normal derivative in Fourier series:

$$
\begin{aligned}
& \theta=\sum_{m=-. M}^{M} a_{m} r^{i m n}
\end{aligned}
$$

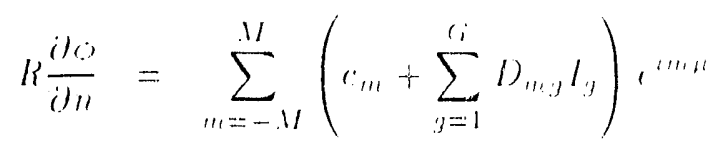


where, in principle, the summation over $m$ should range from $-\infty$ to $+\infty$. Practical considerations, however, require that the Fourier series be truncated after a finite number of terms given by $m= \pm M$.

The coefficients $c_{m}$ and $D_{m g}$ are assumed known. They can be calculated from the Fourier transform of equation 2.43 , and are given by

$$
\begin{aligned}
c_{m} & =\frac{i m}{2 \pi} \int_{0}^{2 \pi}\left(\mu_{0} I_{p} \Psi_{p}+\mu_{0} I_{p} \Psi_{F}\right) e^{-i m \mu} d \mu \\
D_{m g} & =\frac{i m}{2 \pi} \int_{0}^{2 \pi} \mu_{0} \Psi_{g} e^{-i m \mu} d \mu
\end{aligned}
$$

The goals of this analysis are to first find the coefficients $a_{m}$ and then the values of the $I_{I}$ by minimizing $\epsilon_{I}$. The $a_{m}$ are determined by using the expansions given by equations 2.44 and 2.45. These expansions are substituted into equation 2.28 , which is then multiplied by $e^{-i m \prime^{\prime} \prime \prime}$ and integrated over $\mu$. The end result of this process is

$$
\begin{aligned}
& a_{m}+\frac{a_{m}}{\pi} \sum_{m^{\prime}=-M}^{M} \int_{0}^{2 \pi} \int_{0}^{2 \pi} R^{\prime} \frac{\partial\left(r\left(\mu, \mu^{\prime}\right)\right.}{\partial n^{\prime}},{ }^{\prime m} n^{\prime} \mu^{\prime}-i m n d \mu d \mu^{\prime} \\
& =\left(c^{\prime} m+\sum_{g=1}^{C_{i}} D_{m g} I_{y}\right) \sum_{m^{\prime}=-M}^{M} \frac{1}{\pi} \int_{0}^{2 \pi} \int_{0}^{2 \pi}\left(c^{\prime}\left(\mu, \mu^{\prime}\right) f^{i m^{\prime} \mu^{\prime}-i m \mu} d \mu d \mu^{\prime}\right.
\end{aligned}
$$

The integrands on the left and right sides of this equation can be expressed as

$$
\begin{aligned}
& A_{m m^{\prime}}=\frac{1}{\pi} \int_{0}^{2 \pi} \int_{0}^{2 \pi} R^{\prime} \frac{\partial\left(i\left(\mu, \mu^{\prime}\right)\right.}{\partial n^{\prime}} \epsilon^{i m n^{\prime} \mu^{\prime}-i m \mu} d \mu d \mu^{\prime} \\
& C^{\prime} m m^{\prime}=\frac{1}{\pi} \int_{0}^{2 \pi} \int_{0}^{2 \pi}\left(i\left(\mu, \mu^{\prime}\right) \epsilon^{\prime \prime n^{\prime} \mu^{\prime}-i m \mu} d \mu d \mu^{\prime}\right.
\end{aligned}
$$

where $\mathrm{A}$ and $\mathrm{C}$ have dimension $(2 . H+1) \times(2 . M+1)$. With these definitions, equation 2.48 can be expressed in a linear algebraic form:

$$
\{\mathrm{I}+\mathrm{A}] \cdot \mathbf{a}=\mathrm{C} \cdot[\mathbf{c}+\mathrm{D} \cdot \mathrm{i}\}
$$

where $I$ is the identity matrix and $\mathrm{i}$ is a vector of length ${ } i$ containing the values of the grouped currents $I_{y}$. The solution to this equation is

$$
a=d+E \cdot i
$$

where

$$
\begin{aligned}
& \mathrm{d}=[\mathbf{I}+\mathbf{A}]^{-1} \cdot \mathbf{C} \cdot \mathbf{c} \\
& \mathrm{E}=[\mathbf{I}+\mathbf{A}]^{-1} \cdot \mathbf{C} \cdot \mathbf{D}
\end{aligned}
$$


Note that the elements $d_{m}$ and $E_{m m^{\prime}}$ are known quantities depending only upon the coil locations, the plasma shape, and the known fixed currents.

Equation 2.52 can then be substituted into equation 2.13 to express the tangential component of the vacuum field as

$$
B_{u^{\prime}}=b^{(F)}(\mu)+\mathbf{b}^{(G)}(\mu) \cdot \mathbf{i}
$$

where $b^{(F)}(\mu$ ) represents the tangential field (at the plasma surface) due to those conductors carrying fixed currents and $\mathrm{b}^{(C)}(\mu)$ is the tangential field from the grouped conductors. Written in terms of 2.53 and 2.54 , these ficlds are

$$
\begin{aligned}
& b^{(F)}(\mu)=\frac{1}{Q}\left(\sum_{m=-M}^{M}\left(i m d_{m} e^{i m \mu}\right)+\mu_{0} \frac{I_{p}}{R} \frac{\partial \Psi_{p}}{\partial n}+\mu_{0} \frac{I_{p}}{R} \frac{\partial \Psi_{F}}{\partial n}\right) \\
& b_{!}^{(c)}(\mu)=\frac{1}{Q}\left(\sum_{m=-M}^{M}\left(i m E_{m g} e^{i m \mu}\right)+\mu_{0} \frac{1}{R} \frac{\partial \Psi_{!}}{\partial n}\right)
\end{aligned}
$$

\subsection{Solution for Group Currents}

Recall that the ultimate goal of this analysis is to find the conductor currents $l_{j}$ in such a way as to minimize the jump in the tangential field across the plasma surface. From equation 2.23 this jump is written as

$$
\tau_{l}(\mathrm{i})=\frac{\int_{S_{p}}\left(B_{p}-B_{u}\right)^{2} d S}{\int_{S_{p}} B_{p}^{2} d S^{5}}
$$

Substituting equations 2.56 and 2.57 into this expression casts the expression for,$/$ into a simpler form:

$$
c_{l}(\mathbf{i})=\mathrm{i} \cdot \mathrm{M} \cdot \mathrm{i}-2 \mathrm{n} \cdot \mathrm{i}+\mu
$$

where $\mathrm{i}$ is a vector of length ( $i$ representing the group currents being solved for, $\mathrm{M}$ is a $\left({ }^{\prime} i \times(i)\right.$ symmetric matrix, $\mathrm{n}$ is a vector of length $(i$, and $p$ is a scalar. The quantities $M, \mathrm{n}$, and $p$ are, in turn, defined as

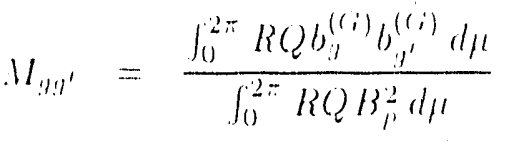

$$
\begin{aligned}
& n_{n}=\frac{\int_{0}^{2 \pi} R Q b_{1}^{((i)}\left(B_{p}-b^{(f)}\right) d \mu}{\int_{0}^{2 \pi} R Q B B_{p}^{2} d \mu} \\
& \mu=\frac{\int_{01}^{2 \pi} R\left(Q\left(B_{p}-b^{(1)}\right)^{2} d \mu\right.}{\int_{11}^{2+} R(2) B_{p}^{2} d \mu}
\end{aligned}
$$


The tangential field error given by equation 2.58 can now be minimized with respect to the group currents. This is done by formally computing the derivative of $c 1$ and setting it to zero:

$$
\frac{\partial \epsilon_{I}}{\partial I_{y}}=0 ; \quad g=1, \ldots, C_{r}
$$

Applying equation 2.62 to equation 2.58 results in a simple linear system given by

$$
\mathrm{M} \cdot \mathrm{i}=\mathrm{n}
$$

This system can be solved through standard matrix techniques [22]. In typical tokamak configurations, $(i$ ranges from to 10 , so equation 2.63 presents a trivial computational task for current computer systems. Finally, it should be observed that the entire calculation is carried out without iteration or searching, implying that this technique will be extremely fast and robust.

Once the vector $\mathrm{i}$ is found, it can be substituted into Equation 2.58 to compute the value of $\%$. This value of $t$ represents an absolute estimate of the error in the radial pressure balance from using this set of coil currents to support the given equilibrium.

\subsection{Summary}

Although the calculations described in this chapter are fairly complex, they can be summarized in a relatively simple manner. The solution procedure assumes that the following information is known:

- Equilibrium information:

1. The plasma shape.

2. The total plasma current.

3. The tangential component of the plasma magnetic lield at the plasma surface, denoted $B_{p}$.

- Conductor information:

1. The locations $(R, Z)$ of the fonductors surrounding the plasma.

2. The currents in $F$ of the $J$ conductors (i.e., the conductors carrying fixed preprogrammed currents) 
3. The grouping of the conductors carrying the same current (i.e., the multifilament model of a finite size coil).

These quantities are the inputs to the solution procedure. The output consists of the $\epsilon_{i}$ minimizing values of the $i$ group conductor currents. These group currents are calculated as follows. First, the plasma shape information is used to compute the matrices $\mathrm{A}$ and $\mathrm{C}$ in equations 2.49 and 2.50 . The plasma shape information is combined with the conductor information to compute the $c$ vector and the $\mathrm{D}$ matrix in equations 2.46 and 2.47 . These matrices are then used to compute the vacuum field surrounding the plasma, $B_{1}$. Substituting $B_{v}$, and the plasma equilibrium field $B_{p}$ into equations $2.59-2.61$ gives $\mathrm{M}$ and $\mathrm{n}$. The group currents $\mathrm{i}$ are then found by solving equation 2.63 . 


\section{Chapter 3}

\section{Asymmetric Systems}

The model developed in Chapter 2 was implemented by Hancy in a program called cPFC. This software takes the data about a given equilibrium and generates a set of poloidal ficld coil currents which support that equilibrium. Haney's version of the program, however, is applicable only to equilibria and coil sets which are up-down symmetric. Thus, the results produced are not applicable to coil sets with asymmetries, single-null diverted equilibria, or systems with current applied to the vertical positioning coils. In this chapter, the original version of the software is extended to include general asymmetric cases.

\subsection{Discussion}

In Chapter 2, the theory behind the original coil current solver was presented. Other than the toroidal axisymmetry requirement, that derivation made no assumptions about plasma or conductor symmetries. In particular, all of the Fourier transforms used to determine the coil currents were expressed in complex form, thereby allowing any plasma-coil configuration to be solved.

To simplify the implementation of this scheme, it was assumed that both the plasma and the coils were symmetric in ez. An example of such a system is shown in Figure 3-1, and the corresponding langential component of the poloidal field is shown in Figure 3-2. Note that the poloidal field is symmetric about $\mu=\pi$.

By assuming that the plasma and coil set were symmetric, it was only necessary to work with the $x$ components of the various Fourier series and transforms presented in Chapter 2. For 


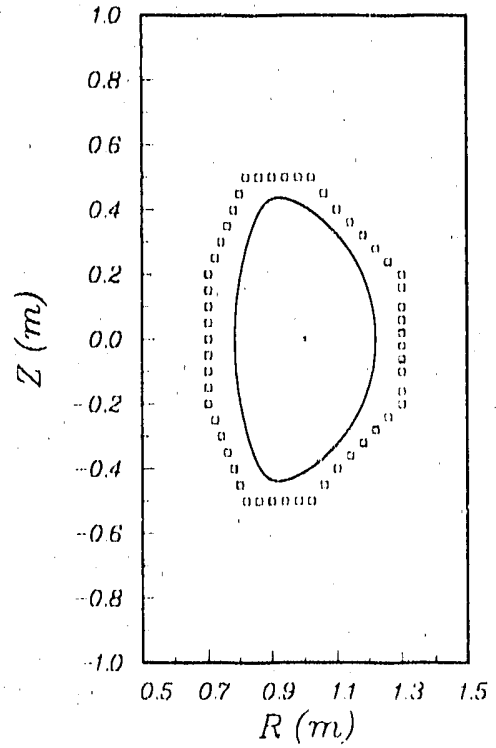

Figure 3-1: Example of a symmetric plasma and coil set

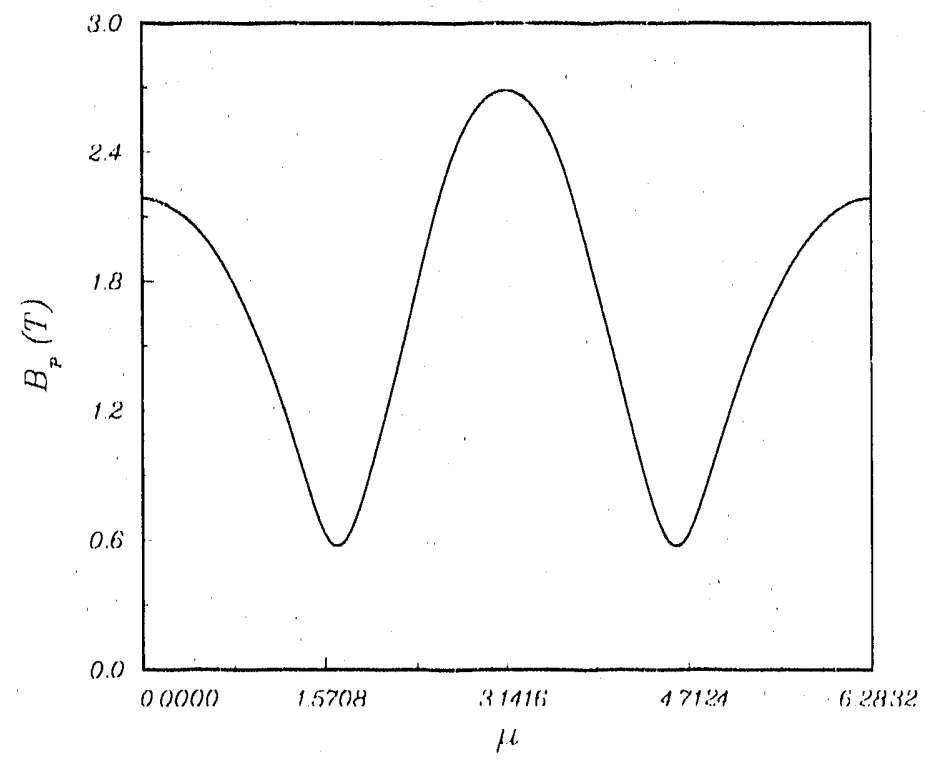

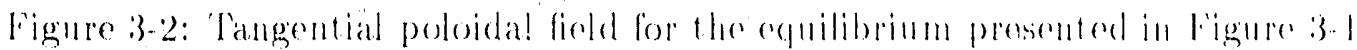


example, in expressing the scalar magnetic potential as a Fouricr serics, Equation 2.44

$$
\phi(\mu)=\sum_{m=-M}^{M} a_{m} e^{i m \mu}
$$

was programmed in the form

$$
\phi(\mu)=\sum_{m=-M}^{M} a_{m n} \cos (m \mu)
$$

where the $i_{m}$ were assumed to be purely real. The other arrays and vectors used to compute the scalar potential were treated in a similar manner. Such treatment is warranted since the Fourier transform of a symmetric (i.c. even) function contains only real components $|18|$.

\subsection{Generalization}

Consider now the single null diverted plasma shown in Figure 3-3. The poloidal field due to this plasma is shown in Figure 3-4. As this last figure clearly shows, the poloidal ficld is no longer symmetric about $\mu=\pi$, making it impossible to find a set of coil currents to support that equilibrium using the original (symmetric) version of (PFC.

Generalizing CPFC to the asymmetric case proved fairly straightiorward. First, all of the real vectors and matrices used to compute the scalar potential and its normal derivative on the plasma surface were converted to complex form. Then, a suitable matrix inversion routine was applied to determine $\mathrm{d}$ and $\mathrm{E}$, which in turn were used to compute a. Finally, the equations for the fixed and grouped components of the vacuum field were explicitly written in the form

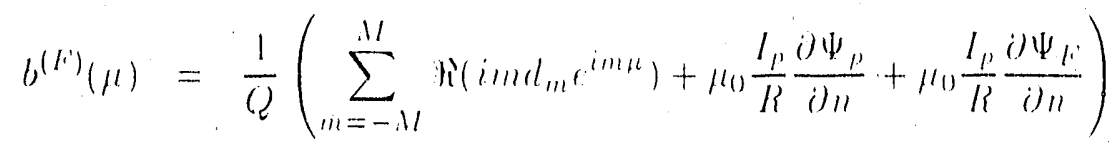

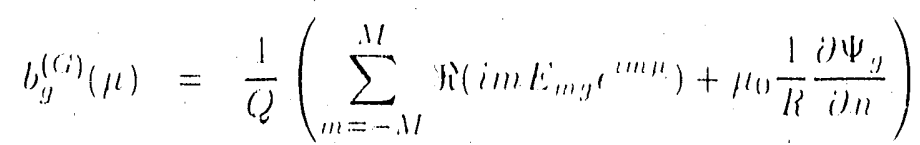

where $t$ is the real operator.

Since the magnetic field is purely real, one test of the accuracy of this transformation is to compute the quantilies

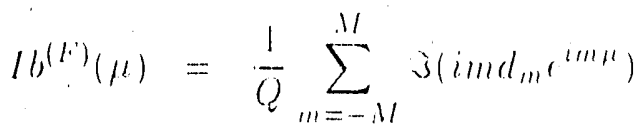

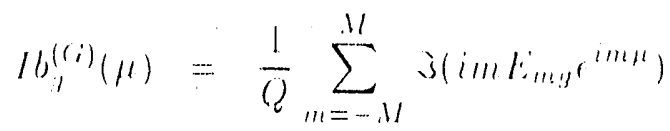




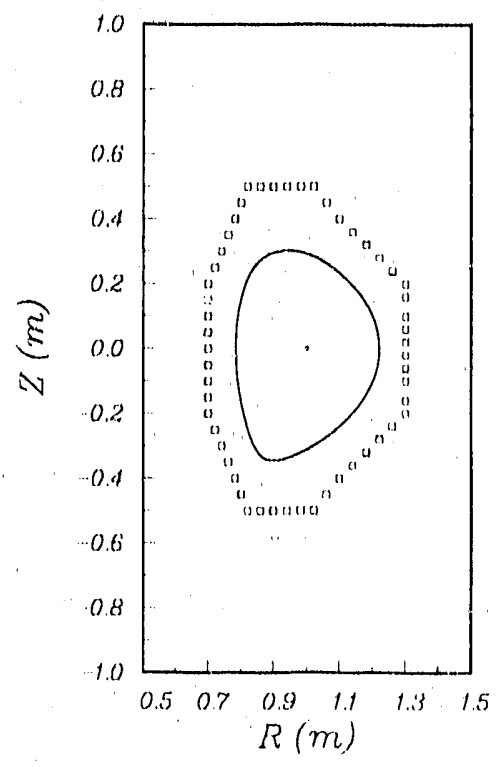

Figure 3-3: Example of a single null diverterl plasma.

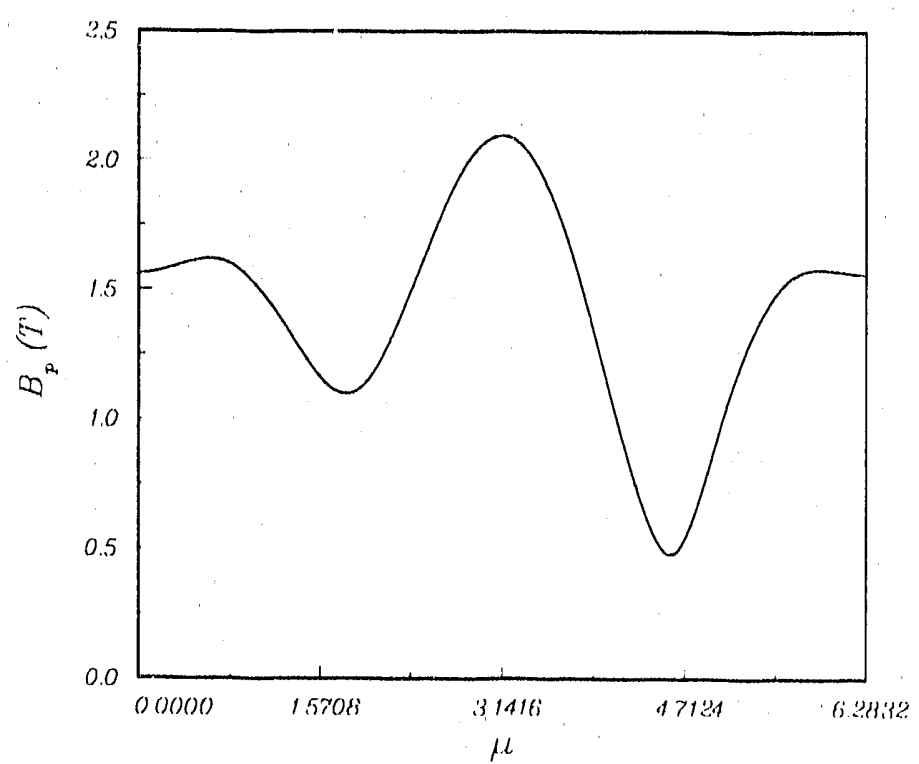

Figure 3-.1: Tangential polodal field of the single mull plasma shown in ligunde 33.3 
where is is the imaginary operator. For a purely symmetric case, these two quantities should be zero. In practice, these values come out is to 10 orders of magnitude less than their real components for the purely up-down symmetric systems presented in the next section and in Appendix A

\subsection{Verification}

With the required modifteations completed, it is necessary to verily the now version of the CPFC program. Here, the first verification process is a consistency check against the symmetric version of the program 1211. If everything has been done properly, the results oblained from the symmetric and asymmetric versions of the cPFC code should be identical for symmetrical coil sets and plasmas. This is the case since, as mentioned previously, the Fourier transform of a symmetric function has only real values.

The first verification process presented here consists of testing three different tokamak configurations. For brevity, only one case is given in this chapter, and the others are presented in Appendix A. Each sample configuration is tested in two modes, one where the coils are grouped up-down (i.e. a symmetric pair of coils is lored to carry the same current), and one where each eoil in the system is allowed to carry a different current. This provides two checks of the system. First, the currents found using the symmetric and asymmetric versions of the cPFC program should be identical in each mode. Second, since the conligurations are perfectly up-down symmetric, the currents found for the grouped and ungrouped modes should also be identical.

Figure 3-5 shows the plasma and coil set of a D shaped tokamak based on the proposed upgrade of the Versalor tokamak al MIT. The plasma parameters for this particular case are given in Table 3.1. The coils labeled OHT4, OHB4, OHTS, and OHB5 all carry a lixed current of 15 kiloamps. The remaining coil currents were determined using both the symmetric and asymmetric versions of ePFC. The data for the grouped mods, is presented in Table 3.2, while the data for the ungrouped mode is listed in Table 3.3. Finally, as a comparison of how well the equilibrium poloidal field is reproduced by the coil currents, this dalla is shown in Figure 3-6. The value of $1 /$ for these currents is (3.().1, showing that the combination of the coil and plasma currents reproduce the equilibrium poloidal field reasonably well.

It is readily apparent that the data for both the symmetric and asymmetric versions of the cPFC code are identical for both the grouped and ungrouped modes. Similar results are found lor 


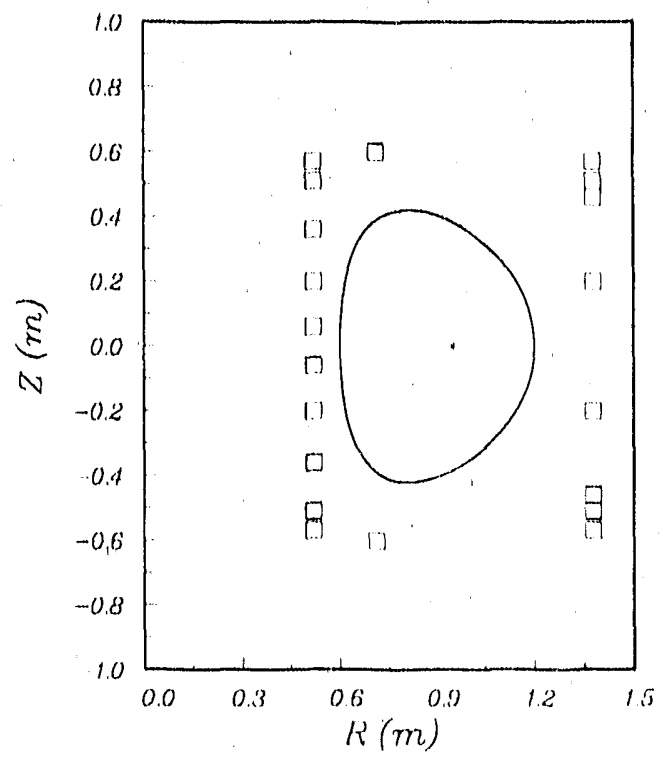

Figure 3-5: Plasma and coil set for the propomed Versator upgrade

Table 3.1: Fiquilibrium parameters used for the Versator equilibrium.

\begin{tabular}{|c|c|}
\hline Palremeter & lislur \\
\hline $\mathrm{R}_{0}(\mathrm{~m})$ & $0.9(0)$ \\
\hline$a(m)$ & 0.300 \\
\hline$\delta$ & $(0.300)$ \\
\hline$h$ & $1.4(0)$ \\
\hline $\mathrm{B}_{0}(\mathrm{~T})$ & $1.0(0)$ \\
\hline$I_{\mu}(k A)$ & $15.0(0)(0)$ \\
\hline 3 & 1.0:31 \\
\hline $3,(\%)$ & (0.69:? \\
\hline
\end{tabular}


Table 3.2: Data for coil groups OHTL-OHT3 and OHBL-OHB3, EFTL and ERBL, LFT2 and EFB2, and EFT3-EFT5 and EFB3-EFBs carrying symmetric currents.

\begin{tabular}{|c|c|c|c|c|c|}
\hline & Symmetric & Asymmetric & & Symmetric: & Asymimetric \\
\hline Coil & \multicolumn{2}{|c|}{ Current (kA) } & Coil & \multicolumn{2}{|c|}{ Current (kA) } \\
\hline OHT1 & -2.131 & -2.131 & $\mathrm{OHB} !$ & -2.131 & -2.131 \\
\hline OHT2 & -2.131 & -2.131 & $\mathrm{OHB} 2$ & -2.131 & -2.131 \\
\hline OHT3 & -2.131 & -2.131 & OHB3 & -2.131 & -2.131 \\
\hline EFT1 & -1.327 & -1.327 & EFB 1 & -1.327 & -1.327 \\
\hline EFT2 & -9.388 & -9.388 & $\mathrm{EFB2}$ & -9.388 & -9.388 \\
\hline EFT3 & -14.824 & -1.1 .82 .1 & EFB3 & -1.4 .82 .1 & -14.824 \\
\hline EFT4 & -1.4 .424 & -14.821 & EFB4 & $-1+1.82 .4$ & -1.1 .82 .1 \\
\hline \multirow[t]{2}{*}{ EFT'5 } & $-1.4 . \times 24$ & -1.1 .82 .1 & EFB5 & -1.1 .12 .21 & -1.1 .82 .1 \\
\hline & \multicolumn{2}{|c|}{ Error $(\%)$} & & . & \\
\hline Field Fimor & 0.04137 & 0.04137 & & & \\
\hline
\end{tabular}

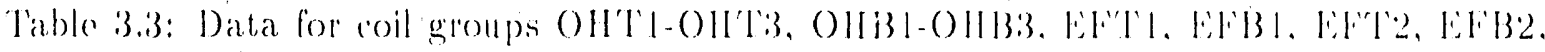

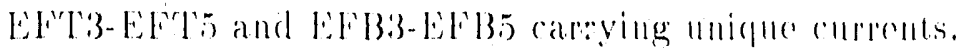

\begin{tabular}{|c|c|c|c|c|c|}
\hline & Siymmentrie & Asymmmetrier & & Symmotrir & Asymmmer tric \\
\hline Coil & \multicolumn{2}{|c|}{ Current (kA) } & Coil & \multicolumn{2}{|c|}{ Current (kA) } \\
\hline OHT1 & -2.131 & $-2.1: 31$ & $\mathrm{OHBl}$ & -2.131 & $-2.1: 31$ \\
\hline OHT2 & $-2.1: 31$ & $-2.1: 31$ & $\mathrm{OHB} 2$ & -2.131 & -2.131 \\
\hline OHT3 & $-2.1: 31$ & $-2.1: 31$ & $\mathrm{OHB} 3$ & -2.131 & -2.131 \\
\hline EFT 1 & -1.327 & -1.327 & EFB I & -1.327 & $-1.32 \pi$ \\
\hline EFT2 & -9.385 & -0.385 & EFB2 & -9.386 & $-9.38 \mathrm{~s}$ \\
\hline EFT3 & $-11 . x 21$ & $-11 . x 21$ & EFB3 & $-11 . x 2 \cdot 1$ & $-11.52 \mid$ \\
\hline EFT4 & -1.1 .424 & -1.4 .122 .1 & $\mathrm{EFB} 4$ & $-11 . \times 2.1$ & -1.1 .2 .1 \\
\hline \multirow[t]{2}{*}{ EFT5 } & $-11 . \times 2 \mid$ & -1.1 .12 .1 & EFB.5 & -||$. \$ 2 \mid$ & $-\mid 1.521$ \\
\hline & \multicolumn{2}{|c|}{ Error $(\%)$} & & & \\
\hline Firld Error & 0.04137 & 0.04137 & & & \\
\hline
\end{tabular}




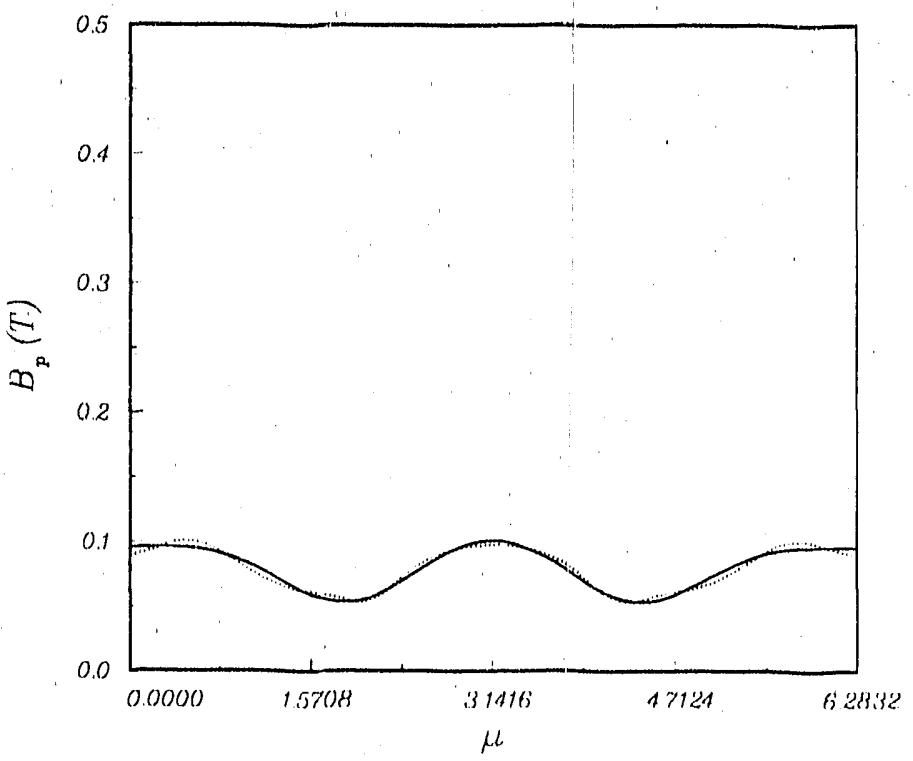

Figure 3-6: Tangential magnetic fiede as a function of "h. The solicl line corresponds to the tangential field at the plasma stiffare, while the doted line represents the best fit possible using this coil set and algorithm. 
the two cases presented in Appendix A. This supplies verification that the asymmetric version is correct with respect to symmetric plasmas and coil sets. The second step is to test and validate the asymmetric version using an asymmetric system.

\subsection{Validation}

Validation of the asymmetric version of the program is accomplished using the method described and used by Haney [14]. This method uses several filaments to model the current distribution within the plasma, thereby climinating the problem of trying to compute an equilibrium using a method which may not be entirely suitable for the given set of poloidal field coils.

The current distribution in the plasma is modeled using a number of filaments. These filaments and those representing the poloidal field coils are then fixed at some appropriate (but essentially arbitrary) values. When the fluxes due to all of these coils are summed, a number of closed flux surfaces are found. By summing the fields from all of these conductors, it is a straightforward matter to compute the tangential ficld on these closed surfaces. The information about the shape of one particular closed flux surface, the tangential field at that surface, and the total current carried by the filaments representing the plasma is then given to cPFC. The currents found by CPFC can then be compared to those originally used to generate the flux surface. Small differences between the currents used to generate the flux surface and the currents produced by CPFC can be expected due to rounding errors and the finite number of terms used in the Fourier series. Large differences would indicate that the method fails to compute the coil currents correctly.

This procedure wili be carried out for two plasma models: one for a symmetric model to demonstrate the method, and one for an asymmetric model to test the asymmetric version of cPFC. Both plasma models are placed within the same coil set, namely a crude model of the Alcator C-Mod coil set.

The symmetric plasma model is shown in Figure 3-7. Note that the eight filaments representing the plasma are symmetrically distributed about the $z$ axis. The triangular shanc reasonably models the effects of a double-null divertor configuration.

The locations of the plasma filaments and the currents carried by those filaments are listed in Table 3.4. The currents carried by the poloidal lield coils and their locations are given in Table 3.5. The results of running the ePFC program on this system are presented in Table 3.6. For this calculation, each coil was allowed to carry a unique current. The Difference column 


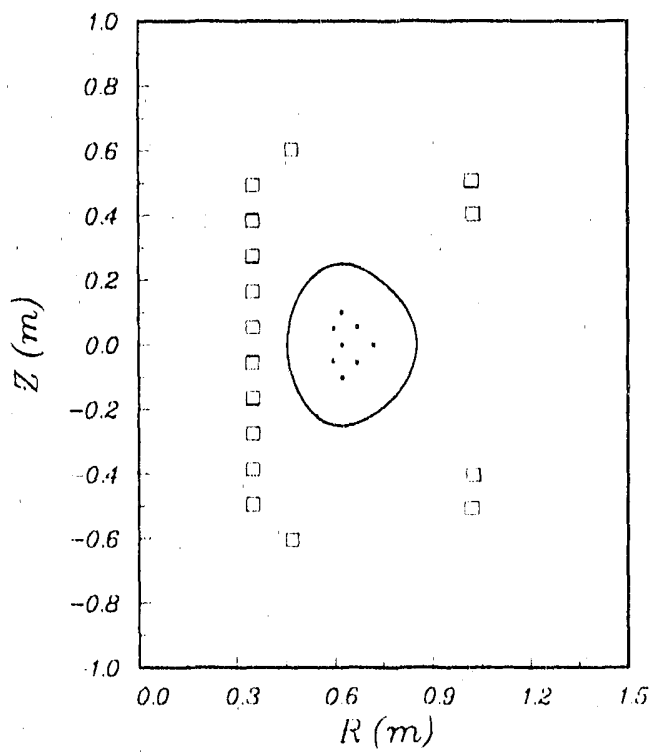

Figure 3-7: Symmetric filament moded of plasma current

Table 3.t: Input plasma filament curents for the symmetric test cass

\begin{tabular}{|c|c|c|c|}
\hline Label & $\mathrm{R}(\mathrm{m})$ & $\mathrm{Z}(\mathrm{m})$ & Current (MA) \\
\hline Plasmal & 0.6190 & $+(0.00(0)$ & $-1.0(000)$ \\
\hline Plasma2 & 0.7190 & +0.0000 & -0.1000 \\
\hline Plasma $3 a$ & 0.5950 & $+0.050(0)$ & -0.1000 \\
\hline Plasma3b & $(0.59 .50$ & -0.0 .500 & -0.1000 \\
\hline Plasma4a & $0.66(5 \times 0)$ & $+(0.055)$ & $-0.70(00)$ \\
\hline Plasma $4 b$ & 0.66680 & -0.05550 & $-(0.7000$ \\
\hline Plasma5a & $0 .(j 190)$ & $+0.1010)$ & $-0.10(0)$ \\
\hline Plasma5b & 0.61190 & $-(0.1010$ & -0.1000 \\
\hline
\end{tabular}


Table 3.5: Input poloidal field coil currents for cPFe' validation

\begin{tabular}{|c|c|c|c|}
\hline Label & $\mathrm{R}(\mathrm{m})$ & $\mathrm{Z}(\mathrm{m})$ & Current (MA) \\
\hline PFla & 0.4690 & +0.6040 & -0.587() \\
\hline PFIb & 0.1690 & $-0.6(0) 10$ & -0.5870 \\
\hline PF2a & $1.0200)$ & +0.5060 & $+0.9: 3.10$ \\
\hline $\mathrm{PF} 2 \mathrm{~b}$ & 1.0200 & -0.5060 & +0.933410 \\
\hline PF3a & 1.0250 & $+0.10: 30$ & +0.933 .10 \\
\hline PF3b & 1.0250 & $-(0.40: 30$ & $+0.9 .3 \cdot 10$ \\
\hline OHla & 0.35500 & $+(0.0 .5 .50$ & $+(0.99900$ \\
\hline $\mathrm{OH} 1 \mathrm{~b}$ & $0.3 .50(0)$ & $-(0.0550)$ & $+(0.99900$ \\
\hline $\mathrm{OH} 2 \mathrm{a}$ & 0.35 .500 & +0.1650 & $+0.990(0)$ \\
\hline $\mathrm{OH} 2 \mathrm{~b}$ & 0.35000 & $-0.16 i 50$ & $+(0.99000$ \\
\hline $\mathrm{OH} 3 \mathrm{a}$ & 0.35000 & +0.27 .10 & $+(0.999(0)$ \\
\hline $\mathrm{OH} 3 \mathrm{~b}$ & $(0.35500$ & $-(0.27 \cdot 10$ & $+(0.99(0) 0$ \\
\hline $\mathrm{OH} 4 \mathrm{a}$ & 0.35500 & +0.35 .10 & +0.99900 \\
\hline $\mathrm{OH} 4 \mathrm{~b}$ & 0.3500 & $-0.3 \times 10$ & $+(0.99900$ \\
\hline $\mathrm{OH} 5 \mathrm{a}$ & 0.35000 & $+0.19 \cdot 10$ & $+(0.99(9)()$ \\
\hline OHSb & 0.3 .500 & -0.19 .10 & $+(0.9)(0)(0)$ \\
\hline
\end{tabular}


Table 3.6: (omparison between input and output values of the poloidal tield coil currents used for the symmetric test case.

\begin{tabular}{|c|c|c|c|}
\hline Label & $\begin{array}{c}\text { Input } \\
\text { Current (MA) }\end{array}$ & $\begin{array}{c}\text { Output } \\
\text { Current (MA) }\end{array}$ & Difference $(\%)$ \\
\hline PFla & -0.5870 & -0.5868 & $+0.02 \cdot 180$ \\
\hline PF1b & -0.5870 & -0.58699 & $+0.02 \cdot 167$ \\
\hline$P F 2 a$ & $+0.93 \cdot 40$ & $+0.933: 39$ & $+0.00(69) 1$ \\
\hline PF2b & $+(0.93: 10$ & $+(0.93339$ & $+0.00(69.3$ \\
\hline PF3a & +0.93340 & +0.93340 & $-0.0(0 .+20$ \\
\hline PF3b & $+(0.933110$ & +0.933 .40 & $-(0.0(0) 419$ \\
\hline $\mathrm{OH} 1 \mathrm{a}$ & $+(0.99000$ & +0.98999 & +0.00137 \\
\hline $\mathrm{OH} 1 \mathrm{~b}$ & $+(0.990(0)$ & +0.9899 & +0.00136 \\
\hline $\mathrm{OH} 2 \mathrm{a}$ & $+(0.99000$ & +0.98999 & +0.001332 \\
\hline $\mathrm{OH} 2 \mathrm{~b}$ & +0.99000 & +0.9899 & $+0.0(01: 3: 2$ \\
\hline $\mathrm{OH} 3 \mathrm{a}$ & $+(0.99900)$ & $+(0.98999$ & $+0.00021: 3$ \\
\hline $\mathrm{OH} 3 \mathrm{~b}$ & $+(0.99900$ & $+(0.98999$ & $+0.0(0) 211$ \\
\hline $\mathrm{OH} 4 \mathrm{a}$ & $+0.990(0)$ & +0.99001 & -0.013337 \\
\hline $\mathrm{OH} 4 \mathrm{~b}$ & +0.9900 & $+(0.990) 1$ & $-0.013: 325$ \\
\hline $\mathrm{OH} 5 \mathrm{a}$ & $+(0.990(0)$ & +0.98966 & $+0.03: 37.16 j$ \\
\hline $\mathrm{OH} 5 \mathrm{~b}$ & $+(0.99900$ & +0.98966 & $+0.0: 372.1$ \\
\hline
\end{tabular}

Table 3.7: Input plasma filament currents for the asymumetric test catse

\begin{tabular}{|c|c|c|c|}
\hline Iabel & $\mathrm{R}(\mathrm{m})$ & $\mathrm{Z}(\mathrm{m})$ & Current (MA) \\
\hline Plasinal & 0.61190 & +0.00000 & $-1.35(7)$ \\
\hline Plasma2 & 0.7190 & +0.00000 & $-0.1500)$ \\
\hline Plasmaza & $(0.59 .50$ & $+0.05(0)$ & -0.1000 \\
\hline Plasma3b & 0.5950 & -0.0 .500 & $-0.2 .50(0)$ \\
\hline Plasma4b & 0.666800 & -0.05500 & $-0.750)$ \\
\hline Plasma5b & $0 .(j ! 90)$ & -0.1010 & -0.1000 \\
\hline
\end{tabular}




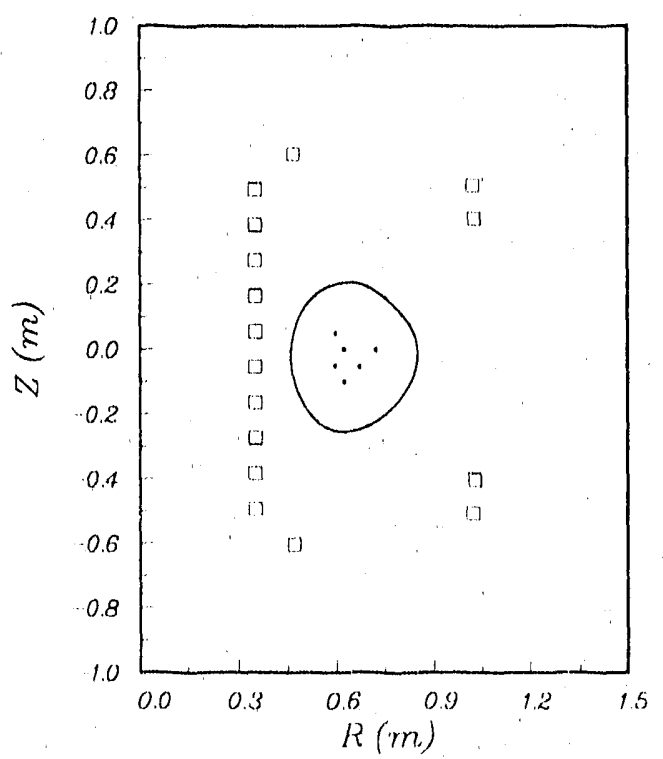

Figure 3-9: Flux surface for the asymmetric case

and resulting flux surface for this case. Here, the triangularity in the lower half of the flux surface models a single-null divertor.

As for the symmetric case, the results obtained by running $\mathrm{PFC}$ for this case indicate that the program is computing the coil currents correctly. This can be verified by examining Table 3.8 . Here, the worst case differs from the input current by $0.614 \%$ (coil OH5a). This is a factor of twenty higher than the symmetric case, but is still acceptable as proof that cPFC is finding the coil currents properly. This can also be seen by examining Figure 3-10, which shows both the poloidal field at the flux surface and the resulting poloidal field computed by CPFC. The field error for this case is $1.2 \cdot 10^{-4} \%$, so the two lines in Figure 3-10 are indistinguishable.

As a final example, consider the plasma and coil set shown in Figure 3-11. This equilibrium is far more asymmetric than that used for the previous test. The coil set shown in Figure 3-11 is a more advanced design of the Alcator C-Mod poloidal field coil system. The locations of the wires making up the various coils shown in Figure 3-11 are given in Appendix B. The equilibrium used in this case is characterized by the parameters given in Table 3.9. The poloidal fields for this case are shown in Figure 3-12. Examining this figure shows that cPFC is doing a reasonable job of reproducing the equilibrium ficld, with a field error of $5.6(9.3 \% \%$. 
Table 3.8: Comparison between input and output values of the poloidal field coil currents used for the asymmetric test case.

\begin{tabular}{|c|c|c|c|}
\hline Label & $\begin{array}{c}\text { Input } \\
\text { Current (MA) }\end{array}$ & $\begin{array}{c}\text { Output } \\
\text { Current (MA) }\end{array}$ & Difference $(\%)$ \\
\hline PF1a & -0.5870 & -0.58 .19 & $-(0.35024$ \\
\hline PF1b & -0.5870 & -0.5876 & -0.10126 \\
\hline PF2a & $+(0.033 .10$ & +0.933336 & -0.04135 .34 \\
\hline PF2b & $+0.9: 340$ & +0.933 .10 & $+0.0086(9)$ \\
\hline PF3a & +0.9340 & +0.933 .12 & +0.02 .1666 \\
\hline PF3b & +0.933410 & $+(0.933399$ & $-0.0(070.1$ \\
\hline $\mathrm{OH} 1 \mathrm{a}$ & +0.99000 & $+0.9899 ?$ & $+0.007(61$ \\
\hline $\mathrm{OH} 1 \mathrm{~b}$ & $+(0.99000$ & $+(0.98999$ & $+0.00(5.595$ \\
\hline $\mathrm{OH} 2 \mathrm{a}$ & $+0.990(0)$ & $+0.990(0)$ & $-0.0(0.289$ \\
\hline $\mathrm{OH} 2 \mathrm{~b}$ & $+(0.99000$ & $+(0.98999$ & $+0.00 \pi(6 s$ \\
\hline $\mathrm{OH} 3 \mathrm{a}$ & +0.99000 & +0.98933 & $+0.06(589) !$ \\
\hline $\mathrm{OH} 3 \mathrm{~b}$ & +0.9900 & $+0.99(0)(0)$ & $-0.0(0): 3.15$ \\
\hline $\mathrm{OH} 4 \mathrm{a}$ & $+0.99(00)$ & +0.99933 & -0.33355 .2 \\
\hline $\mathrm{OH} 4 \mathrm{~b}$ & $+(0.99(0)$ & +0.98993 & +0.071222 \\
\hline $\mathrm{OH} 5 \mathrm{a}$ & +0.9900 & $+0.9 \times 3.39$ & $+0.031: 3(3.1$ \\
\hline OH5b & $+(0.990(0)$ & +0.99111 & $-0.1159)$ \\
\hline
\end{tabular}




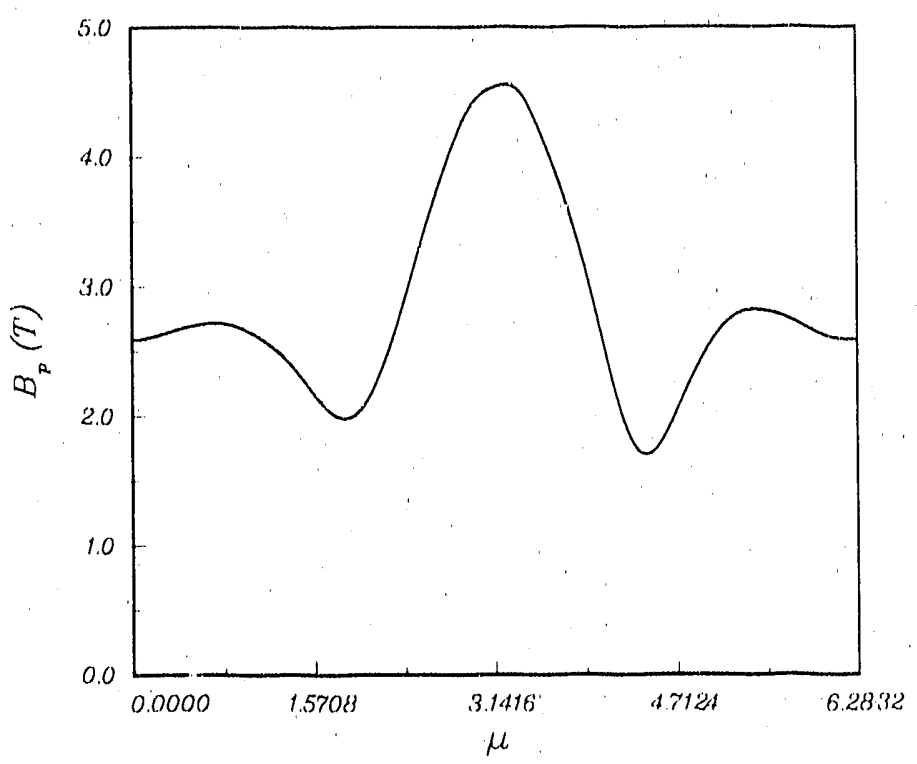

Figure 3-10: Plot of the tangential component a the poloidal field for the asvinmetric test case. The solid line represents the field due to the plasma. The indistinguishable dotterl line is the field due to the plasma and the PF coils.

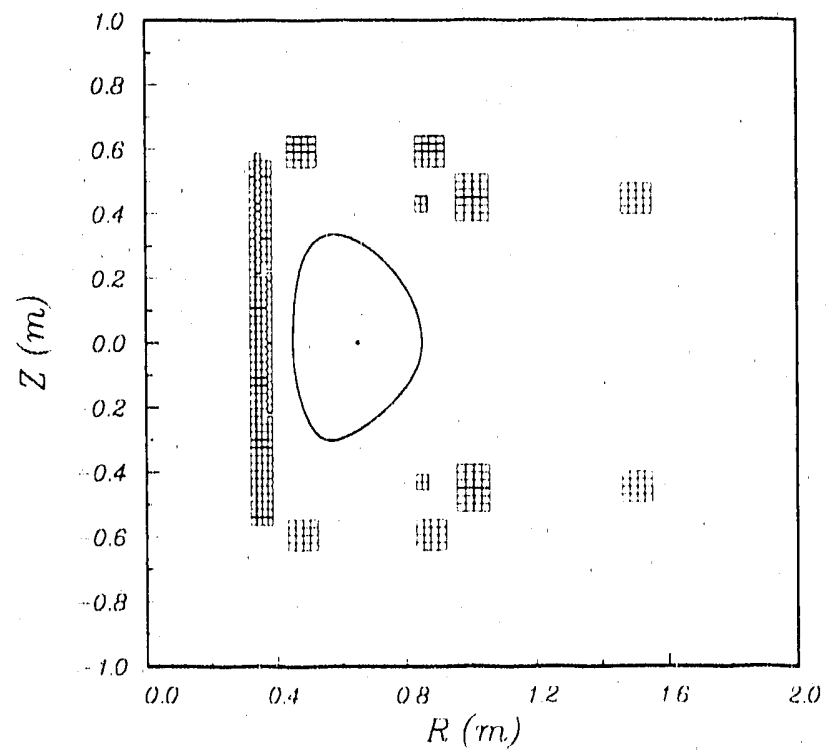

Figure 3-11: A single null diverted plasma and the Alator ('-Model coil set 
Table 3.9: Equilibrium parameters for the Alcator single null equilibrimm.

\begin{tabular}{|c|c|}
\hline \hline Parameter & Value \\
\hline $\mathrm{R}_{0}(\mathrm{~m})$ & 0.650 \\
$\mathrm{a}(\mathrm{m})$ & 0.210 \\
$\delta_{u}$ & 0.384 \\
$\delta_{l}$ & 0.446 \\
$\kappa_{u}$ & 1.708 \\
$\kappa_{l}$ & 1.55 .5 \\
$\mathrm{~B}_{0}(\mathrm{~T})$ & 0.000 \\
$\mathrm{I}_{p}(\mathrm{MA})$ & $2.500)$ \\
$\beta_{p}$ & 0.292 \\
$\beta_{l}(\%)$ & 1.070 \\
\hline \hline
\end{tabular}

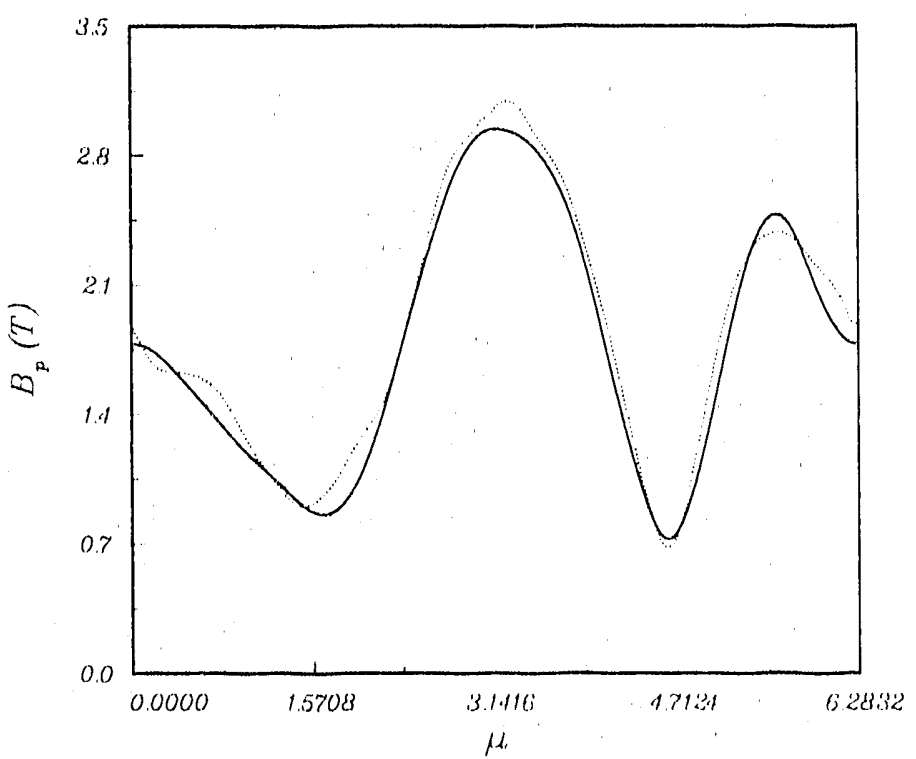

Figure 3-12: Poloidal fields for the ('-Mod single null test ase. The solid line is the tangential component of the poloidal field due to the plasma. The dotted line reperesents the combined fields of the plasma and the ple coil set. 


\section{Chapter 4}

\section{Linear Constraints}

The previous chapter discussed the transformation of the cPFC program into a general purpose tool for computing the coil currents required to support a given equilibrium. In this chapter, linear limits on ti coil currents are incorporated into CPFC. These limits arise from enginecring considerations for the poloidal field coils. The chapter begins with a discussion of the engineering factors that limit the current that a coil can carry.

\subsection{The Source of the Constraints}

The way in which current limits arise depends on the type of magnet being used. In resistive magnets, the coil temperature is one limiting factor on the magnitude of the current, while superconducting magnets have tenuperature, magnetic field, and current limits that must be satisfied. The details of these limits are examined in the next two sections.

\subsubsection{Resistive Magnets}

Linear constraints on the coil currents of a system of resistive magnets come about due to temperature considerations. As a demonstration of how the maximum allowable tenuperature in a coil limits the current flowing in that coil, consider a long, straight resistive wire with a circular cross section. The maximum temperature allowed in the wire is defined to be $\%$. The amount of power $(P)$ deposited as heat in the wire is given by

$$
l^{\prime}=I^{2} R l
$$


where $R$ is the wire's resistance per unit length, $L$ is the total length of the wire and $l$ is the current llowing through the wire.

Knowing the power dissipated in the wire makes it possible to determine the temperature distribution of the wire from

$$
\kappa \nabla^{2} T+P=\rho c^{\prime} p \frac{\partial T}{\partial \tau}
$$

where $\tau$ is time, $k$ is the thermal conductivity of the wire, $p$ is the wire's density and $c^{\prime} p$ is the specific heat of the wire. For simplicity, $k, \rho$ and $c_{p} p$ are assumed to be independent of temperature. Furthermore, assuming a steady state condition removes the time dependence from this equation, resulting in

$$
\nabla^{2} T=-\frac{P}{R}
$$

The general solution to this Equation [19] is given by

$$
T=\frac{\dot{q}}{4 r}\left(r_{0}^{2}-r^{2}\right)+T_{s}
$$

where $r$ is the radial position, $r o$ is the radius of the wire, ' $T_{s}$ is the temperature at the surface of the wire $\left(r=r_{0}\right)$, and $\dot{q}$ is the volumetric heat generation, given by

$$
\dot{q}=\frac{P}{\pi r_{0}^{2} L}
$$

The temperature distribution described by Equation 4.1 is parabolic and has a maximum at $r=0$, corresponding to the center of the wire.

The surface temperature of the wire is found by applying an appropriate boundary condition. For this case, the wire is assumed to be undergoing convective heat transfer with a cooling medium. The heat transfer rate to the coolant (c) is given by

$$
C=h A\left(T_{s}-T_{b}\right)
$$

where $h$ is a heat transfer coefficient, $A$ is the surface area over which cooling is taking place, $T_{s s}$ is the temperature of the wire's surface, and $T_{b}$ is the bulk temperature of the coolant. The previous assumption of being at steady state gives

$$
I^{2} R I=h \cdot A\left(T_{s}^{\prime}-T_{1}\right)
$$

This equation yields $T_{s,}^{\prime}$, which can then be substituted into Equation 4.1 to obtain the temperature at the ecenter of the wire $\left(T_{c}^{\prime}\right)$. The equation for $T_{1}^{\prime}$, is

$$
T_{i}=\frac{P}{4 \pi L i t}+T_{s}^{\prime}=I^{2} R\left(\frac{1}{1 \pi h}+\frac{L}{h \cdot d}\right)+T_{b}
$$


As discussed earlier, the maximum temperature occurs at the center of the wirc. For the current case, this means that $T_{c}$ must be less than or equal to $T_{0}$. This translates into

$$
I^{2} R \leq \frac{4 \pi h_{i} h\left(T_{0}-T_{i}\right)}{h_{A} A+4 \pi \kappa_{i} L}
$$

The maximum current in the wire is then found by taking the square root in the previous equation:

$$
I_{m a t}=\left[\frac{1}{R}\left(\frac{4 \pi r h A\left(T_{0}-T_{b}\right)}{h A+4 \pi r L}\right)\right]^{1 / 2}
$$

Thus, the current allowed to flow through the coil due to the temperature considerations is given by $-I_{m a x} \leq I \leq I_{m a x}$. The upper and lower bounds on $I$ act as a constraint, and since the exponent of $I$ is unity, the constraint is linear.

\subsubsection{Superconducting Magnets}

Superconducting magnet materials exhibit a complex hehavior in the phase space defined by current density, magnetic field, and temperature. The critical values of these parameters are defined as the point at which the superconducting nature of the material is lost. Since a superconductor has essentially no electrical resistance, Ohmic heating is not a factor in the current limits. Instead, the temperature of the superconductor can change through internal energy fluctuations or nuclear interactions. For the purposes of this discussion, the critical current will be assumed to be the only limiting factor to the coil current.

Present designs for superconducting magnets consist of a serics of superconducting filaments embedded in a metallic substrate. The substrate serves as a backup in case part of the superconductor fails temporarily due to a local temperature increase and acts as a heat sink. Consider a magnet composed of Niobium-Tin filaments which have a circular cross-section. At $4.2 \mathrm{~K}$ and in a $5 \mathrm{~T}$ magnetic field, the critical current density for Niobium-Tin is approximately $5 \cdot 10^{9} \mathrm{~A} / \mathrm{m}^{2}$. If the total filamentsave a cross-sectional area of $5 \mathrm{~cm}^{2}$, the maximum current the magnet can carry is $100 \mathrm{kA}$. Thus, in a grossly oversimplified situation, the critical current acts as the limiting factor on the current that a superconducting magnet can carry.

\subsection{Problem Statement}

As presented in Chapter 2, the problem of finding the poloidal field coil currents was simply that of minimizing the functional

$$
r(\mathrm{i})=\mathrm{i}^{T} \cdot \mathrm{M} \cdot \mathrm{i}-2 \mathrm{n}^{T} \cdot \mathrm{i}+\mu
$$


Since $\mathrm{M}$ is a symmetric matrix, this problem reduces to finding the solution of

$$
\mathrm{M} \cdot \mathbf{i}=\mathbf{n}
$$

Now, assume that a particular current $i_{k}$ is subject to a maximum current constraint of the form

$$
\left|i_{k}\right| \leq b_{k}
$$

More generally, if a finite subset of the group currents is subject to a set of inequality constraints, the constraint equations take the form

$$
-b \leq 1 \leq b
$$

where 1 denotes the enstrained subset of the grouped currents. The problem of finding the coil currents subject to the linear constraints is formally expressed as

$$
\begin{aligned}
\text { Minimize } & \mathbf{i}^{1 T} \cdot \mathbf{M} \cdot \mathbf{i}-2 \mathbf{n}^{T} \cdot \mathbf{i}+l \\
\text { Subject to } & -\mathbf{b} \leq \mathbf{1} \leq \mathbf{b}
\end{aligned}
$$

Equation 4.6 is commonly referred to as an optimization problem. To simplify the discussion, some of the nomenclature involved in optimization problems is now presented [9]. Any set of currents $\mathrm{i}$ that satisfy the constraints defined by Equation 4.5 represents a frasible point. A point $\mathrm{i}$ which satisfies all of the constraints and minimizes $\&$ is called optimal. For constraints involving inequalities, a satisfied constraint is called incetive and one which is violated is described as active. In general, solving a problem with inequality constraints is difficult. This difficulty arises because at an 8 step in the solution process, none, some, or all of the constraints may be active, so that it is impossible to tell a prior" which of the inequalities ultimately appear as constraints.

There are a variety of problems associated with the determination of an optimal point of a quadratic system with linear inequality constraints - precisely the problem expressed in Equation 4.6. The worst of these is the issue of convergence. Since one of the goals of this work is to build a fast, robust system, a system which may not converge to one set of $\mathrm{i}$ is undesirable. Rather than face these problems, the goal of minimizing ${ }_{/}(\mathrm{i})$ subject to the constraints is simplified to that of finding a feasible point. This is accomplished by assuming that if the global minimum found in Equation 4.4 does not satisfy the constraints, then a feasible poirt will not perturb the system too far from the global minimum, with the field error serving as an estimate of the quality of the solution. The formulae for estimating a leasible point are developed in the next section. 


\subsection{Finding a Feasible Point}

The goal of this section is to find a feasible point of Equation 4.6. To do this, an intermediate expression for Equation 4.6 is developed using the Lagrange multiplier technique. The result can then be treated in a fairly straightforward manner.

Originally, the problem simply required finding a minimum of the expression

$$
\epsilon_{l}(\mathbf{i})=\mathbf{i}^{T} \cdot \mathbf{M} \cdot \mathbf{i}-2 \mathbf{n}^{T} \cdot \mathbf{i}+p
$$

The presence of linear inequality constraints transformed this problem to the problem stated by Equation 4.6. Since each constraint is independent of the others, Equation 4.6 can be replaced by the auxiliary function [16]

$$
\varphi(\mathbf{i})=\mathbf{i}^{T} \cdot \mathbf{M} \cdot \mathbf{i}-2 \mathbf{n}^{T} \cdot \mathbf{i}+p+\lambda^{T} \cdot \mathbf{g}
$$

where $\lambda$ is a vector of Lagrange multipliers aid $\mathrm{g}$ is a vector whose elements are defined by the constraint equations:

$$
g_{n}=l_{n}-b_{n}
$$

Here, 1 is the constrained subset of $i$. 'The combination of Equation 4.7 and the constraint equations form a linear system which can be minimizcd. This is accomplished by setting the derivative of Equation 4.7 with respect to $\mathrm{i}$ equal to zero and imposing the constraint equations. The first three terms on the right-hand side of Equation 4.7 can simply be replaced by $c_{l}(\mathrm{i})$, whose derivative with respect to $i$ is known, resulting in

$$
\frac{\partial \varphi}{\partial \mathrm{i}}=\mathrm{M} \cdot \mathbf{i}-\mathbf{n}+\frac{\lambda^{T}}{2} \cdot \frac{\partial \mathrm{g}}{\partial \mathrm{i}}
$$

The derivative of $\mathrm{g}$ can be found using

$$
\frac{\partial y_{n}}{\partial i_{j}}=\delta_{n, j}
$$

where $\delta_{n, i}$ is a selection function defined as

$$
\delta_{n, j}= \begin{cases}1 & \text { if } l_{n} \text { corresponds to the current } i_{,} \\ 0 & \text { otherwise }\end{cases}
$$

For $N$ total grouped currents, of which $\Lambda$ are constrained, Equation 4.9 yiclds $N$ equations in $N+\kappa$ unknowns (the $N$ variables $\mathrm{i}$ and the $k$ values of $\lambda$ ). The $l$ constraint equations complete the system, giving $N+h$ equations in $N+h$ unknowns. The next step is to find a method of solving the system in light of the inequalities in the constraint equations. 


\subsection{Numerical Implementation}

Although the equations developed in the previous section appear straightforward to solve, this is not the case because of the inequalities in the constraint equations. The algorithm which is best suited to this case is an adaptation of Newton's method to systems of nonlinear equations [2]. The details of this algorithm are presented in this section.

Newton's method arises from attempting to find a fixed point $x$ for the function $h(x)$ defined by

$$
h(x)=x-\phi(x) f(x)
$$

The fixed point of $h(x)$ is defined as the point $p$ where $h(p)=p$. From this definition, it is obvious that $\phi(p) f(p)=0$. The one dimensional version of Newton's method arises from the desire to find a functional form for $\phi(x)$ with the property that $h(x)$ converges quadratically to the fixed point $p$. Newton's method comes about directly from this condition by choosing $\phi(i x)=1 / f^{\prime}(x)$, provided that $f^{\prime}(p) \neq 0$.

Approaching the $n$-dimensional case in a similar manner involves finding a matrix

$$
\mathbf{A}(\mathbf{x})=\left[\begin{array}{cccc}
a_{11}(\mathbf{x}) & a_{12}(\mathbf{x}) & \cdots & a_{1 n}(\mathbf{x}) \\
a_{21}(\mathbf{x}) & a_{22}(\mathbf{x}) & \cdots & a_{2 n}(\mathbf{x}) \\
\vdots & \vdots & & \vdots \\
a_{n 1}(\mathbf{x}) & a_{n 2}(\mathbf{x}) & \cdots & a_{n n}(\mathbf{x})
\end{array}\right]
$$

such that the equation

$$
H(x)=x-A(x)^{-1} F(x)
$$

gives quadratic convergence to the tixed point of $F(x)=0$, provided that $A(x)$ is nonsingular at the fixed point. The appropriate choice for the matrix $A(x)$ is the Jacobian of $F(x)$. Denoting the Jacobian $\mathbf{J}(\mathbf{x})$, Equation 4.13 becomes

$$
H(x)=x-J(x)^{-1} F^{\prime}(x)
$$

where the Jacobian is determined from [17]:

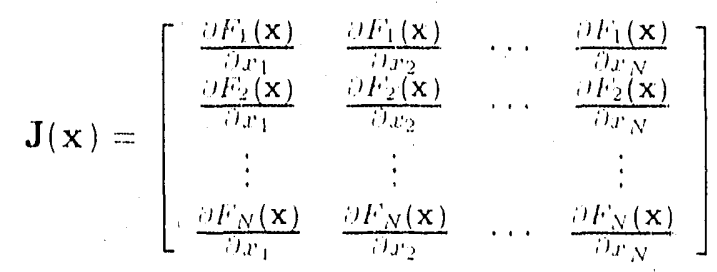


The solution to this system is found by iterating from an initial guess, with the iteration scheme being

$$
\mathbf{x}^{n+1}=\mathbf{x}^{n}-\mathbf{J}\left(\mathbf{x}^{n}\right)^{-1} \cdot \mathbf{F}\left(\mathbf{x}^{n}\right)
$$

Adapting this system to the formulae presented in the previous section is fairly straighilorward. The first $N$ elements of $\mathrm{x}$ represent the grouped currents $\mathrm{i}$ of the system. The remaining $H$ elements are the Lagrange multipliers. From Equation 4.9, the first $N$ equations of the $\mathbf{F}(\mathbf{x})$ vector are of the form

$$
F_{i}(\mathbf{x})=\sum_{j=1}^{N} M_{i j} x_{j}-n_{j}+\frac{1}{2} \sum_{j=1}^{K} \lambda_{j} \frac{\partial g_{j}}{\partial i_{i}}
$$

and the final $K$ equations are given by the constraint equations $g . F(x)$ for the $K$ constraint equations is evaluated from

$$
F_{j}(\mathbf{x})= \begin{cases}l_{j}-b_{j} & \text { if } l_{j}>b_{j} \\ 0 & \text { if }-b_{j} \leq l_{j} \leq b_{j} \\ l_{j}+b_{j} & \text { if } l_{j}<-b_{j}\end{cases}
$$

where $l_{j}$ corresponds to the $k$ th constrained element of $\mathrm{x}$. Finally, the first $N$ rows of the Jacobian matrix are evaluated airectly from Equation 4.17, and the derivatives of the $K$ constraint equations are evaluated from the form given in Equations 4.10 and 4.11.

There are two potential problems to using the iteration scheme defined by Equation 4.16. The first of these is the need to evaluate the Jacobian matrix for each iteration. Careful examination of the equations used, however, shows that the Jacobian is constant for this case, so it only has to be evaluated once. The other problem is that the Jacobian has to be inverted at each iteration. In practice, Equation 4.16 is evaluated in two steps: first

$$
\mathbf{J}(\mathbf{x}) \cdot \mathbf{y}=-\mathbf{F}(\mathbf{x})
$$

is computed, followed by

$$
\mathrm{x}^{n+1}=\mathrm{x}^{n}+\mathrm{y}
$$

where $\mathbf{y}$ is the update vector corresponding to $-\mathbf{J}(\mathbf{x})^{-1} \mathrm{~F}(\mathrm{x})$.

The stopping criterion for this algorithm is simply that the solution has been found when all of the constraints are inactive. This condition indicates that a feasible point has been found.

To summarize, the algorithm for finding the feasible point works in the following manner. First, the unconstrained solution is found. Then, the Jacobian matrix is computed. The Jacobian 
matrix is then used to iteratively solve $\mathbf{F}(\mathbf{x})=0$, with the iteration process terminating when all of the constraints are satisfied.

With an algorithm capable of finding a feasible point of the system given in Equation 4.6, the next step is to test it. But first, it is necessary to go over the derivation just presented and clarify a few points.

\subsection{Avoiding Singularities}

The iteration scheme for Newton's method is based on

$$
x^{n+1}=x^{n}-\frac{f(x)}{f^{\prime}(x)}
$$

This scheme's major downfall occurs when $f^{\prime}(x)=0$, resulting in a singularity. A similar problem occurs with the algorithm given in the previous section. Recall that the constraint equations were expressed as

$$
g_{n}=l_{n}-b_{n}
$$

where 1 is the constrained subset of the coil currents. Rigorously, the constraint equations are

$$
g_{n}= \begin{cases}l_{n}-b_{n} & \text { if } l_{n}>b_{n} \\ 0 & \text { if }-b_{n}<l_{n}<b_{n} \\ l_{n}+b_{n} & \text { if } l_{n}<-b_{n}\end{cases}
$$

The derivative of this expression is

$$
\frac{\partial g_{n}}{\partial i_{j}}= \begin{cases}\delta_{n, j} & \text { if } l_{n}>b_{n} \\ 0 & \text { if }-b_{n}<l_{n}<b_{n} \\ \delta_{n, j} & \text { if } l_{n}<-b_{n}\end{cases}
$$

where $\delta_{n, j}$ is given by Equation 4.11. If the constraint is inactive, the derivative is zero, resulting in a singular Jacobian.

The standard approach to this would be to eliminate the inactive constraint from the system $|8|$. This results in a computationally intensive algorithm which shrinks and expands the system of equations as constraints become inactive or active. This method can also converge to a set of two points, one whers: a constraint is inactive and another where the constraint is active. The iteration scheine would then move from one point to another, never converging. Since the goal of this process is to find a feasible point rather than an optimum, these issues can be avoided entirely. 
The algorithm presented in the previous section should suffice, provided that the singularities can be eliminated or avoided.

The problem of a singular Jacobian can be avoided by using Equation 4.10. Treating the derivative in this manner has no effect on the calculation of the coil currents, as will now be demonstrated.

Consider an inactive constraint equation from the linear system in Equation 4.19. This equation has the form

$$
\frac{\partial g_{j}}{\partial i_{n}} y_{n}=F_{n}(\mathbf{i})
$$

Since the constraint is inactive, $F_{n}(\mathbf{i})=0$. Using Equation 4.24 to cvaluate the derivative would result in the indeterminate solution

$$
y_{n}=\frac{0}{0}
$$

If Equation 4.10 is used, the solution would be

$$
y_{n}=\frac{0}{1}=0
$$

The expected solution is $y_{n}=0$ : the current should not be changed if the constraint is inactive. Finally, using equation 4.10 to evaluate the derivative of $\mathrm{g}$ in Equation 4.17 affects only the calculation of the Lagrange multipliers, not the coil currents. Thus, by using Equation 4.10 the correct results are obtained and the problem of a singular Jacobian is avoided.

\subsection{Verification, Validation, and Testing}

Since the algorithm described in Section 4.5 is invoked only if current constraints are specified, verification of the program only requires ensuring that the results obtained for the cases presented in Section 3.3 and in Appendix A are identical. As expected, the results did match, indicating that the linear constraint algorithm has no global side effects in the cPFC program.

Validation and testing the lincar constraint algorithm is straightforward. It is only necessary to run a set of cases which have varying numbers of constraints and ensure that the results satisfy those constraints. This will be done for the equilibrium and coil set depicted in Figure 4-1. The parameters characterizing this equilibrium are listed in Table 4.1 .

The coil set shown in Figure 4-1 represents the Alcator C-Mod tokamak at MIT. It is composed of 335 wires making up 13 unique coils. Of these, 3 coils carry fixed currents: the $\mathrm{OH} 1$ coil carries a total current of $1.4964 \mathrm{MA}$, the EFC upper coil carries 3.00(0) kA, and the lower EFC 


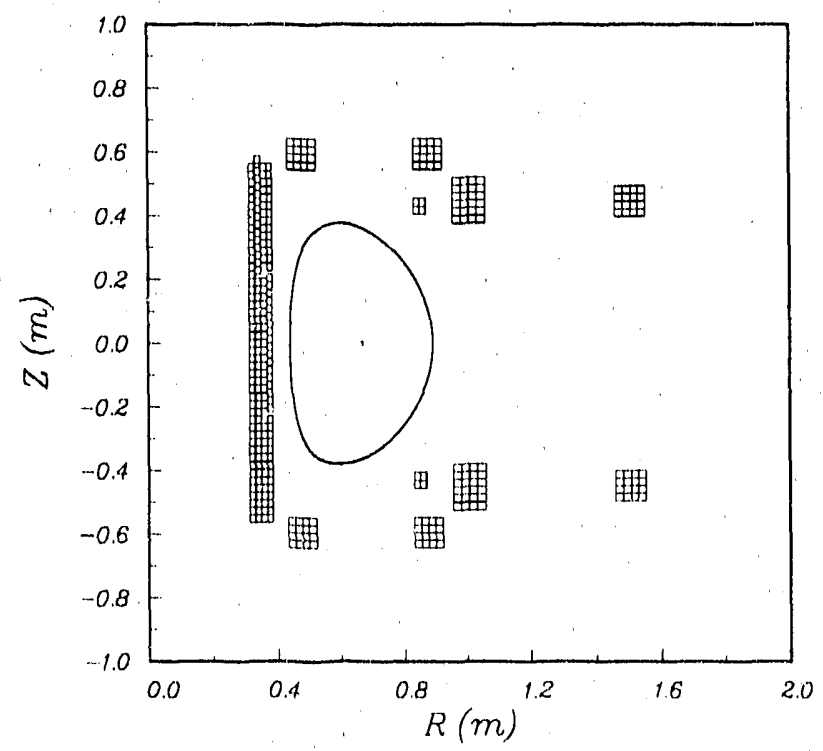

Figure 4-1: The Alcator C-Mod equilibrium and coil set used to validate and test the linearly constrained coil current algorithm.

Table 4.1: Equilibrium data for the Alcator ('-Mod test case.

\begin{tabular}{|c|c|}
\hline \hline Parameter & Value \\
\hline $\mathrm{R}_{0}(\mathrm{~m})$ & 0.665 \\
$\mathrm{a}(\mathrm{m})$ & 0.225 \\
$\delta$ & 0.310 \\
$\kappa$ & $1.6 \%()$ \\
$\mathrm{B}_{0}(\mathrm{~T})$ & 8.500 \\
$\mathrm{I}_{p}(\mathrm{MA})$ & 2.500 \\
$\beta_{p}$ & 0.500 \\
$\beta_{t}(\%)$ & 0.017 \\
\hline
\end{tabular}




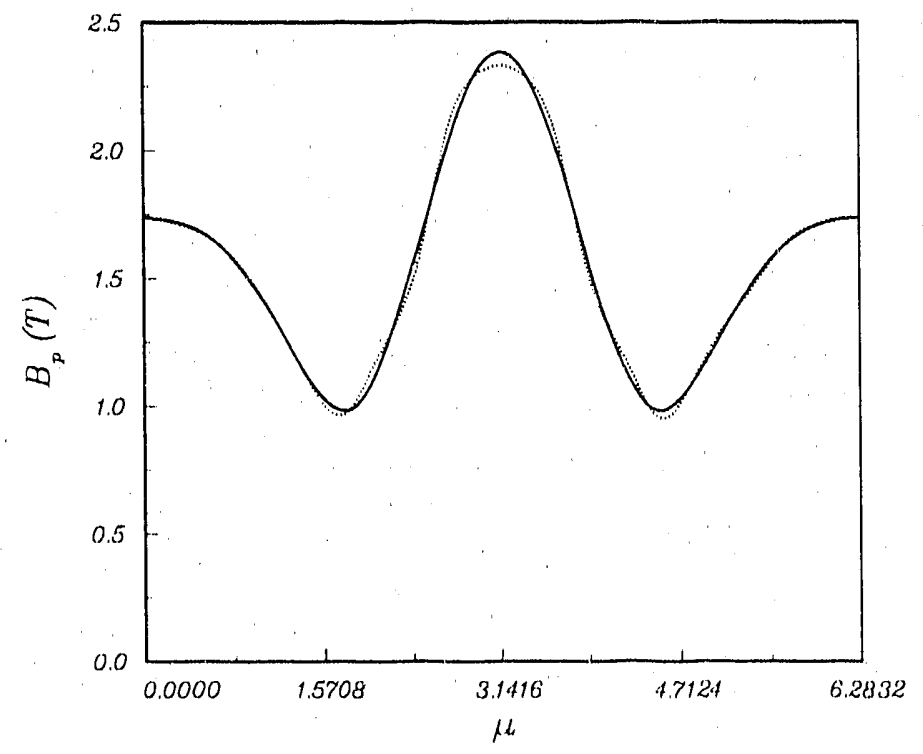

Figure 4-2: Comparison of the tangential components of the equilibrium poloidal ficld (solid line) and that produced by the combination of the plasma and coil set (dotted linc) for the Alcator -Mod test case with no constraints.

coil carries $-3.0000 \mathrm{kA}$. The sign on the currents is relative to the direction of the plasma current, so a negative current is flowing opposite the plasma current. As a reference, locations of the wires making up the various coils are listed in Appendix B. In addition, the initial operating mode has the EF3 and EF4 coils grouped. The results obtained for the unconstrained case are shown in Figure 4-2. The field error for this case is $1.40 .8 \%$.

The first test to be made is to determine if the algorithm of Section 4.5 actually works. Examining the coil currents produced by the unconstrained case (listed with the results of all of the tests in Table 4.2) shows that the current in each wire of the upper EF1 coil is $51.27 \mathrm{kA}$. Limiting this current to $50.00 \mathrm{kA}$ should provide a good test of the system. The results of this test are shown in Figure 4-3. Comparing this figure to Figure 4-2 shows a slight difference in the region of $\mu=\pi / 2$. Since the constraint was set to a value very nearly equal to that prodnecd by the unconstrained case, only slight differences are expected between the two cases. This is further born out by the field error, which for this case is $1.131 \%$, just a little larger than the $1.10 \mathrm{~s} \%$ error produced by the unconstrained case. Finally, examining the results listed in Table 4.2 shows that the constraint has been satisfied. 


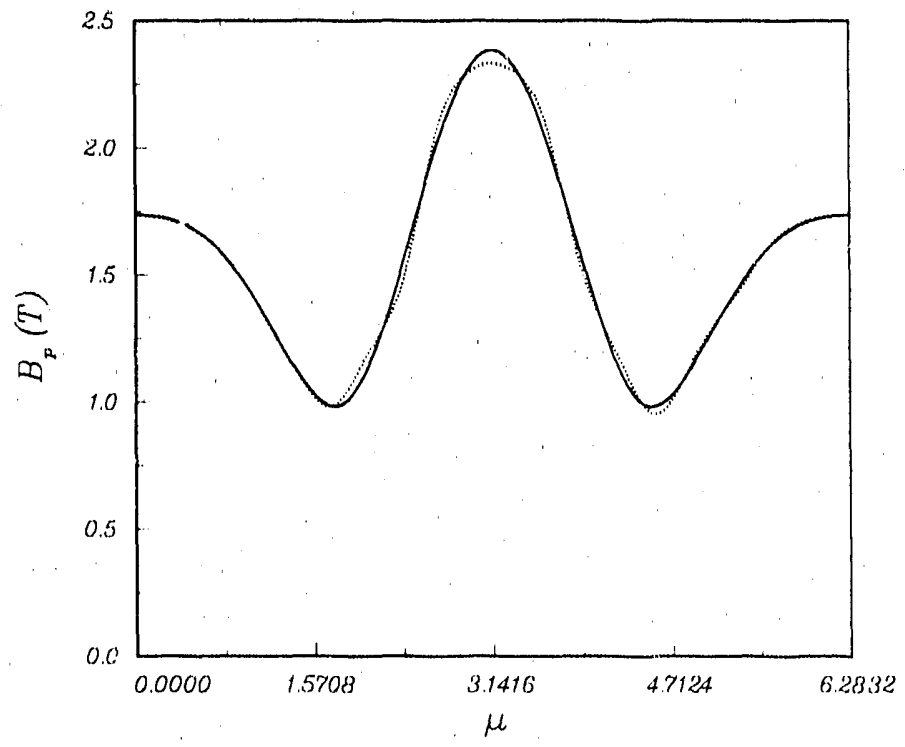

Figure 4-3: Comparison of the equilibrium poloidal field and that produced by the combination of the plasma and coil set when the upper EF(' coil current is constrained to a maximum current of $50.00 \mathrm{kA}$. 


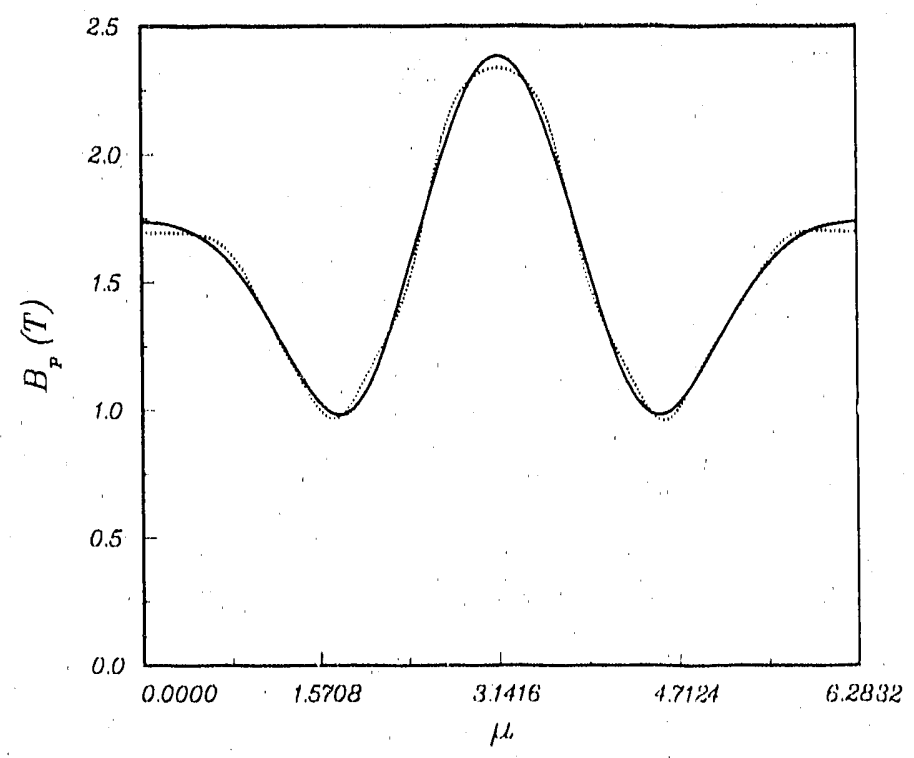

Figure 4-4: Comparison of the equilibrium poloidal field and that produced by the combination of the plasma and coil set when both the upper EFC coil current and the EF't coil currents are constrained to a maximum current of $50.00 \mathrm{kA}$.

The next test is to ensure that multiple violated constraints are satisfied. This is accomplished by setting the maximum current in the EF4 coils to $50.00 \mathrm{kA}$ and retaining the constraint on the EF1 upper coil. This constraint is also violated by the currents found in the unconstrained case. The effects of this constraint are much larger, as can be seen by examining Figure 4-4. The difference between the equilibrium poloidal field and the field produced by the coils is now markedly different in the region around $\mu=0=2 \pi$. The field error for this case is $1.822 \%$.

The next case retains the two constraints just tested, and adds one which is satisfied in the unconstrained case but is violated by the feasible point found in the previous test. For this, the current flowing in the wires of the EF3 coils is limited to a maximum value of $x .35 \mathrm{kA}$. The current in these wires from the unconstrained case is $1.15 \mathrm{kA}$. The tangential poloidal ficld for this test is illustrated in Figure 4-5. The ficld error lor this test is $2.977 \%$.

These results are substantially worse than those of the previous test. If the goal of the constraint solving algorithm was to find an optimal or near optimal point of this system, these results would be unacceptable. Since, however, the goal of the algorithm was to determine a feasible point, these results satisfy that requirement. The reason for this discrepancy will be 


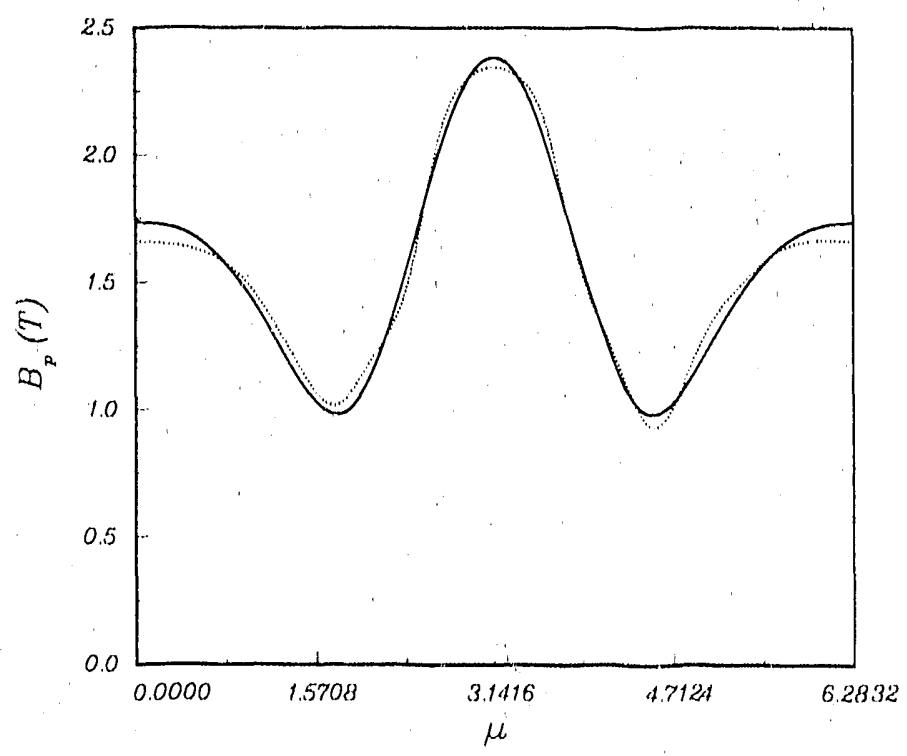

Figure 4-5: Comparison of the equilibrium poloidal ficld and that produced by the combination of the plasma and coil set when both the upper EFC coil current and the EFt coil currents are constrained to a maximum current of $50.00 \mathrm{k}$, and the current in the EF:3 coil is set to a maximum of $8.35 \mathrm{kA}$. 


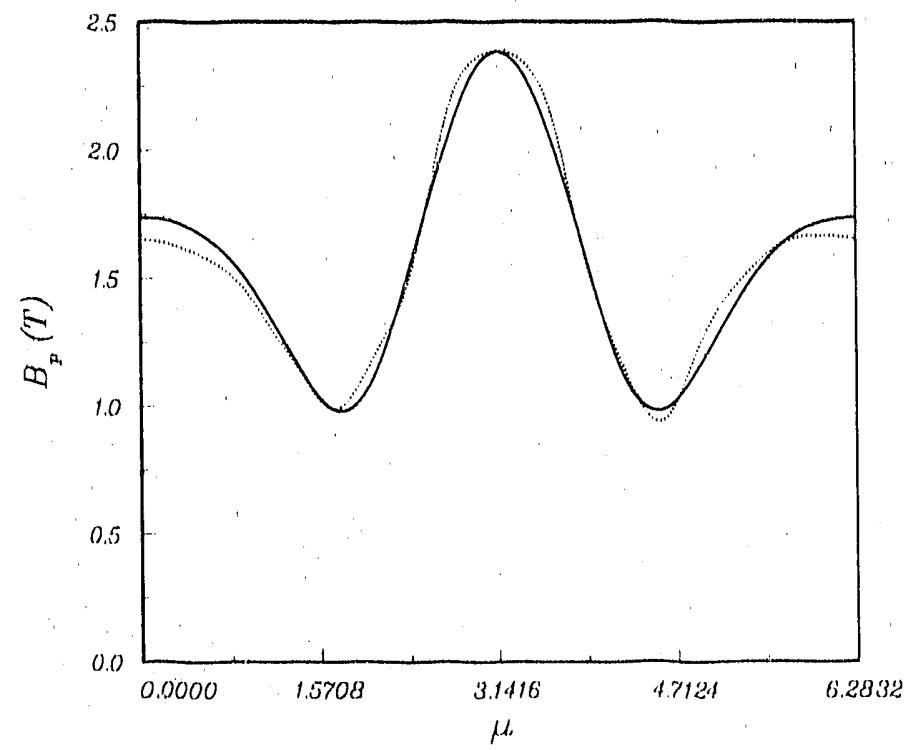

Figure 4-6: Comparison of the equilibrium poloidal field and that produced by the combination of the plasma and coil set when both the upper EF(' coil current and the EFA coil currents are constrained to a maximum current of $50.00 \mathrm{k} \Lambda$, the curent in the bis coil is set to a maximum of $8.35 \mathrm{kA}$, and the current in the upper coil of EF'2 is limited to $100.00 \mathrm{kA}$.

discussed at the end of the present chapter.

The final test to run is an extension of the previous case, namely a test where the unconstrained result violate two constraints and satisfy two constraints. Based on the results of the previous case, the results of this test should be worse. Here, in addition to the three constraints imposed previously, the current in the wires of the EF2 upper coil is limited to a maximum value of $100.00 \mathrm{kA}$. This case yields a field error of $3.715 \%$, and the resulting field is compared with the equilibriurn ficld in Figure 4-6.

The coil currents for these 5 test cases are summarized in Table 4.2.

\subsection{Discussion}

As demonstrated in the previous section, the algorithm presented in Section 4.5 finds a feasible point for the system. However, for inactive constraints, the solution has a larger field error than 
Table 4.2: Summary of the test results for the linear constraint algorithm.

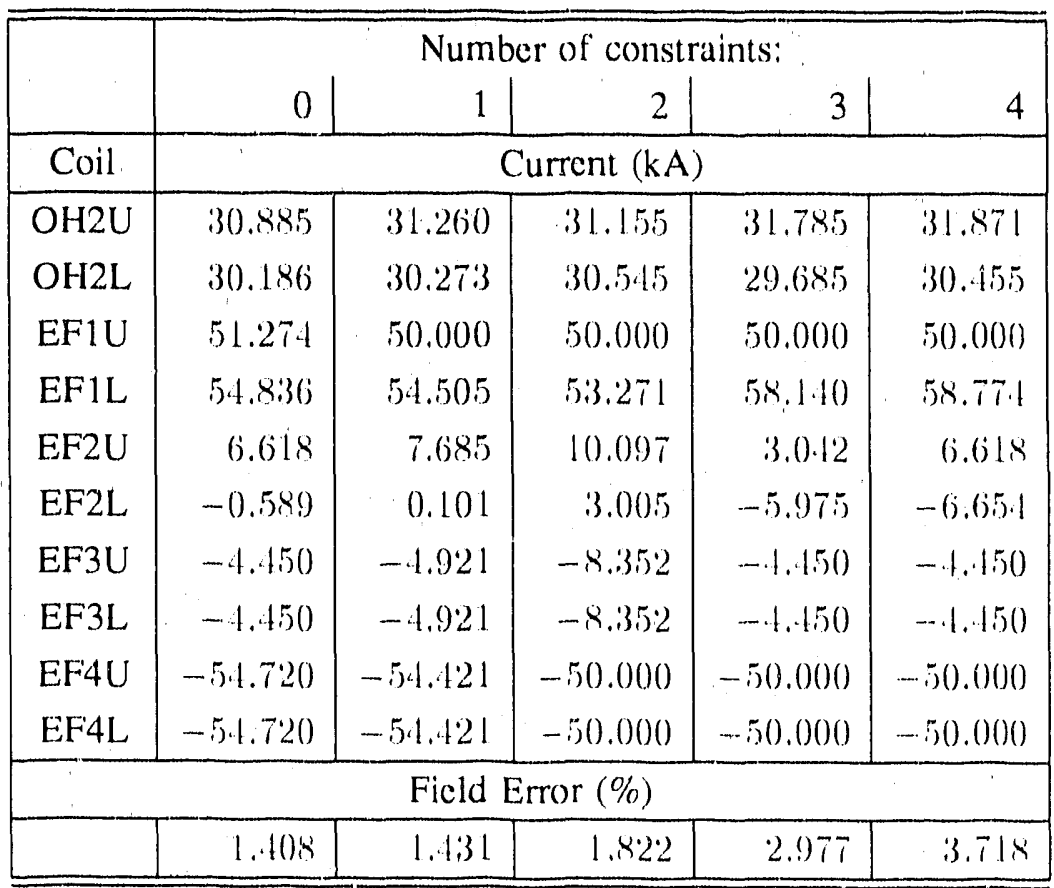


necessary. Specifically, the last three columns of Table 4.2 should be identical.

These undesirable results are directly related to the algorithm. Recall that the update vector $y$ is found from

$$
\mathrm{J}(\mathrm{x}) \cdot \mathrm{y}=\mathrm{F}(\mathrm{x})
$$

If the ith constraint is inactive, then $F_{m}(x)=0$, where $F_{m}$ is the equation corresponding to the $i$ th constraint equation. This sets $y_{m}=0$, which does not allow the current to vary. Since the current does not vary, the constraint is inactive for the next itcration. The net effect of this is that a constraint which is inactive from the global minimization process locks the value of the corresponding current to the value of that current at the global minimum.

With a small number of coils (13 for the case of Alcator C-Mod), a simple method for using the program would be to:

- Run CPFC tor the unconstrained case, and determine which constraint is most violated

- Set the corresponding current to its maximum value and run CPFC again, looking for the next most violated constraint

- Set the current for this constraint to its maximum value, run cPFC with these two constraints and repeat the procedure until no more constraints are violated.

This method avoids the problem of an inactive constraint locking the corresponding current to its initial value, thereby making it possible to find the "best" or near "best" set of currents to support a given equilibrium. Applying this technique to the tests pertormed in the previous section, the user would have stopped after the case with two active constraints. Recall that this case had a field error $(1.522 \%)$ very close to the field error of the unconstrained case $(1.40 \% \%)$. It should be noted that if too many of the constraints are violated by a large margin, even the optimum set of coil currents will not represent a good solution, since the field error will be much larger than desired.

Finally, some mention should be made of the execution times required to obtain these results. The unconstrained case required 0.608.t CPU seconds on a Cray-2 to estimate the currents, and needed a total of 11.07 CPU seconds to run. For the case of two active and two inactive constraints (the final case in the previous section), estimating the currents use 0.(685.1 CPU seconds and a total of $10.99 \mathrm{CPU}$ seconds to run. These results indicate that the implementation of the linear constraint algorithm does not appreciably increase the total amount of CPU time required to run the program. 


\section{Chapter 5}

\section{Forces on PF Coils}

The next set of constraints to be imposed on the coil currents arise from the forces acting on the coils. In this chapter, the equations used to calculate those forces are derived. This forec model will be used in the next chapter to compute the coil currents such that the coil force limits are not exceeded.

The coil-coil interaction forces are derived over the next four sections. These forces are computed from the potential energy of a set of coils. The potential energy, in turn, is computed from the vector potential, which is the subject of the next section.

\subsection{The Vector Potential}

Maxwell's equation for the divergence of a magnetic field $B$ states

$$
\Gamma \cdot \mathrm{B}=0
$$

throughout all space, By making use of the property that

$$
\Gamma \cdot(\Gamma \times \mathbf{A})=0
$$

for any vector $\mathbf{A}$, the magnetic field may be expressed in terms of $\mathbf{A}$ through the relation

$$
\mathrm{B}=\Gamma \times \mathrm{A}
$$

With the magnetic field defined in this manner, $\mathrm{A}$ is referred to as the efelor polentiul of $\mathrm{B}$. 


\subsubsection{Vector Potential of a Distributed Current}

From the Biot-Savart law, the vector potential describing a distributed current (carrying a current density $\mathbf{J}$ ) is given by

$$
\mathbf{A}(\mathbf{r})=\frac{\mu_{0}}{4 \pi} \int_{V^{\prime}} \frac{\mathbf{J}\left(\mathbf{r}^{\prime}\right)}{\left|\mathbf{r}-\mathbf{r}^{\prime}\right|} d \tau
$$

where $V^{\prime}$ is the volume containing the current $\mathbf{J}$. Although this expression appears quite straightforward, substantial manipulation of it is required to obtain a form approp riate to cylindrical systems. Now, consider the case of a coil with a rectangular cross-section (of width 11 , height $h$, major radius $R$, and elevation $Z$ ) carrying a uniform current density $J$. In this case, $\mathbf{J}=/ \mathbf{e}_{\phi,}$, and the vector potential is given by:

$$
\mathbf{A}(\mathbf{r})=\mathbf{e}_{\phi} \frac{\mu_{0} J}{4 \pi} \int_{Z-(h / 2)}^{Z+(h / 2)} \int_{0}^{2 \pi} \int_{R-(w / 2)}^{R+(w / 2)} \frac{\cos \left(\phi^{\prime}-\phi\right) r^{\prime} d r^{\prime} d \phi^{\prime} d z^{\prime}}{\left[r^{2}+r^{\prime 2}-2 r r^{\prime} \cos \left(\phi^{\prime}-\phi\right)+\left(z-z^{\prime}\right)^{2}\right]^{1 / 2}}
$$

where the direction of $\mathbf{e}_{\phi}$ is the $\mathbf{e}_{\phi}$ of the observation point.

With some work, it is possible to perform the integral in $\phi^{\prime}$. Making the transformation $\beta=\phi^{\prime}-\phi$ gives

$$
A_{\phi}=\frac{\mu_{0} J}{4 \pi} \int_{Z-(h / 2)}^{Z+(h / 2)} \int_{-\phi}^{2 \pi-\phi} \int_{R-(w / 2)}^{R+(w / 2)} \frac{\cos (\beta) r^{\prime} d r^{\prime} d / \beta d z^{\prime}}{\left[r^{2}+r^{\prime 2}-2 r r^{\prime} \cos (\beta)+\left(z-z^{\prime}\right)^{2}\right]^{1 / 2}}
$$

This integrand is cyclic in $\beta$ with a period of $2 \pi$, so the limits on the 13 integral can be rewritten as $(0,2 \pi)$, thereby removing all $\phi$ dependence from the vector potential. It is now possible 10 evaluate the $\beta$ integral, resulting in $|4|$

$$
\mathbf{A}(\mathbf{r})=\mathbf{e}_{d} \frac{\mu_{0} J}{4 \pi} \int r^{\prime}\left(i\left(r, z ; r^{\prime}, z^{\prime}\right) d r^{\prime} d z^{\prime}\right.
$$

where

$$
\begin{aligned}
\left(i\left(r, z ; r^{\prime}, z^{\prime}\right)\right. & =\frac{2 A}{r r^{\prime}}\left[\left(1-k^{2} / 2\right) h(k)-E(k)\right] \\
d^{2} & =\left(r+r^{\prime}\right)^{2}+\left(z-z^{\prime}\right)^{2} \\
k^{2} & =4 r r^{\prime} / A^{2}
\end{aligned}
$$

where $K(k)$ and $E(k)$ are the complete clliptic integrals of the first and second kind, respectively.

\subsubsection{Filamentary Currents}

A current $\mathbf{J}$ flowing in a very thin loop can be considered filamentary, so that

$$
\mathbf{J}(\mathbf{r})=I \delta\left(r^{\prime}-R\right) \delta\left(z^{\prime}-Z\right) \mathbf{e}_{\phi}
$$




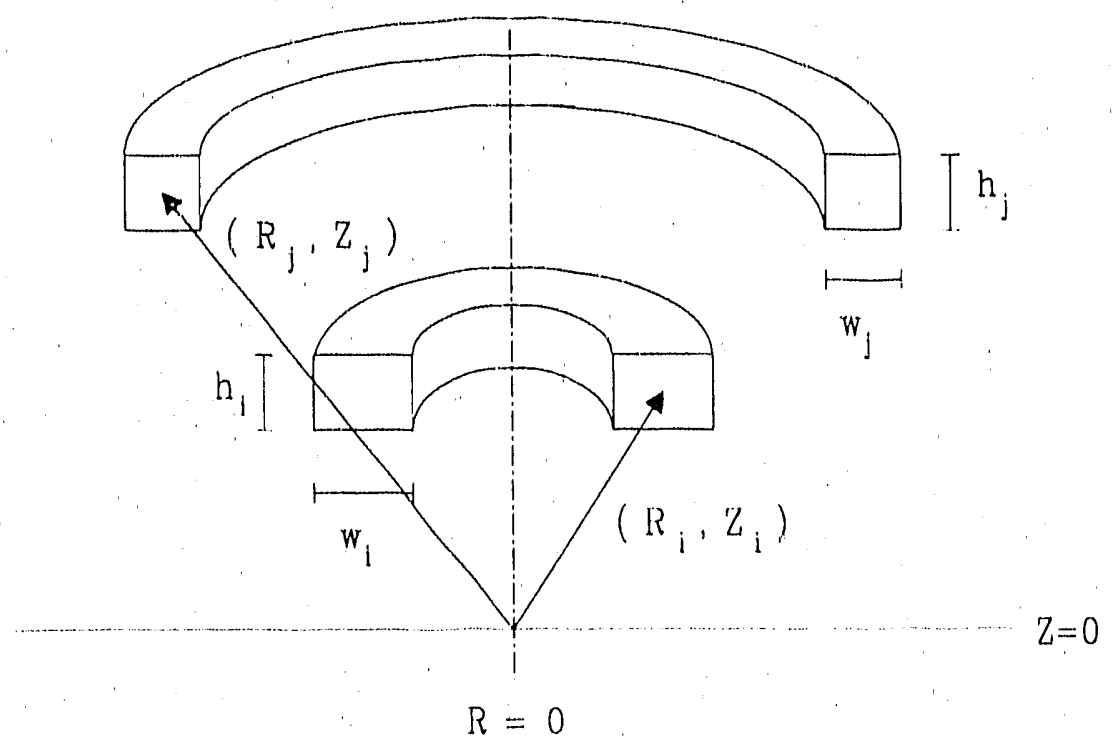

Figure 5-1: Simple coil model for computing the magnetic energy

where $I$ is the total current flowing in the loop and $\delta$ is the Dirac delta function. Using this relation to derive an expression analogous to equation 5.2 results in

$$
\mathbf{A}(\mathbf{r})=\mathbf{e}_{\phi} \frac{\mu_{0} I}{4 \pi} R(r(r, \ldots ; R, Z)
$$

\subsection{Magnetic Energy of a System of Currents}

In this section, the energy of interaction between the coils producing a magnetic field and the currents flowing through those coils will be determined. As in the previous section, a general expression will be developed for distributed currents and a simplification for filamentary currents will follow.

\subsubsection{Two Distributed Currents}

Consider the set of coils shown in Figure 5-1. One coil has height $h_{1}$, radial width ${ }_{1}$, major radius $K_{\imath}$, and elevation given by $\%_{i}$. The characteristics of the other coil are denoted with a subscript $j$. The energy associated with the interaction of $\mathbf{J}_{\mathrm{i}}$ with the magnetic field produced 
by $\mathrm{J}_{\mathrm{j}}$ is found from [25]

$$
U_{j \rightarrow i}=\frac{1}{2} \int_{V} \mathbf{J}_{\mathbf{i}}(\mathbf{r}) \cdot \mathbf{A}_{\mathbf{j}}(\mathbf{r}) d \tau
$$

where $\mathbf{A}_{\mathbf{j}}(\mathbf{r})$ is the vector potential associated with $\mathbf{J}_{\mathbf{j}}$ and $V$ is the volume encompassing $\mathbf{J}_{\mathbf{i}}$.

\subsubsection{A Single Distributed Current}

In addition to interacting with the field produced by all other coils in a system, a current also interacts with its own magnetic field. For this case,

$$
U_{i \rightarrow i}=\frac{1}{2} \int_{V} \mathbf{J}_{\mathbf{i}}(\mathbf{r}) \cdot \mathbf{A}_{\mathbf{i}}(\mathbf{r}) d \tau
$$

\subsubsection{Two Filanientary Currents}

For the case of two filamentary currents, the substitution

$$
\mathbf{J}_{\mathbf{i}}(\mathbf{r})=\mathbf{e}_{\phi} I_{i} \delta\left(r-R_{i}\right) \delta\left(z-Z_{i}\right)
$$

is made in equation 5.7 and equation 5.6 is used for $\mathbf{A}_{\mathbf{j}}(\mathbf{r})$. The resultant expression for the energy is

$$
U_{j \rightarrow i}=\frac{\mu_{0} I_{i} I_{j}}{1} R_{i} R_{j}\left(i\left(R_{i}, Z_{i}: R_{j}, Z_{j}\right)\right.
$$

\subsubsection{A Single Filamentary Current}

Equation 5.9 describes the energy of a set of two filamentary currents. For the case of onc filamentary current, this equation becomes infinite, a clearly unacceptable situation. A filamentary current results in $k=1$ in Equation 5.5, and leads to a singularity in the elliptic integrals in Equation 5.3, making it impossible to find an analytic form for the vector poteuial of a single filament. A suitable approximation can, however, be found by assuming that the coil has a cirrular cross section and then taking the limit as $k$ approaches 1 . In this limit, the vector potential becomes [4]

$$
A_{t \prime \prime}=\frac{\mu_{0} I_{i} R_{i}}{2 \pi}\left[\ln \left(\frac{x R_{i}}{\|}\right)-2\right]
$$

where $a$ is the equivalent radius of the coil, given by

$$
a^{2}=h_{i} \omega_{i} / \pi
$$


The magnetic energy for this coil is given by

$$
U_{i \rightarrow i}=\frac{\mu_{0}}{2} I_{i}^{2} R_{i}\left[\ln \left(\frac{8 R_{i}}{\imath}\right)-2\right]
$$

It will be shown in a later section that approximating a rectangular coil as circular does not adversely affect the results.

\subsection{Force Computations}

Given expressions for the interaction energies of one and two coil systems, it is relatively simple to compute the force un a given coil. This is done by observing how the total energy of the system changes as the coils undergo an infinitesimal displacement from their starting positions. The net force acting on the system can then be written as

$$
\mathbf{F}=-\nabla \sum_{i, j} U_{i, i}
$$

For a two coil system, the total energy is given by

$$
\begin{aligned}
U_{t} & =U_{i \rightarrow i}+U_{i \rightarrow j}+U_{j-i}+U_{1-1} \\
& =U_{i \rightarrow i}+2 U_{i \rightarrow j}+U_{j \rightarrow j}
\end{aligned}
$$

where use has been made of the relation $U_{i-j}=U_{j \rightarrow i}$ as demonstrated in Appendix $\mathrm{C}$.

To compute the force on coil $i$, the $\nabla$ operator is replaced with $\nabla_{\text {, }}$, which is defined as

$$
\nabla_{i}=\mathbf{e}_{\mathbf{R}} \frac{\partial}{\partial R_{i}}+\mathbf{e}_{\mathbf{Z}} \frac{\partial}{\partial Z_{i}}
$$

This operator perturbs the coil about its equilibrium position, giving $\mathbf{F}_{i}$.

As one coil of the two coil system is perturbed, the flux passing through both coils will, in general, change. Conservation of flux, however, requires that the flux passing through each coil remain constant. The next step of the derivation is to find a flux-conserving form for the force expression.

The inductance $U$ of a coil can be expressed in terms of the energy,

$$
H_{i j}=\frac{2 I_{j-i}}{I_{i} I_{j}}
$$

With this equation, it is possible to express the llux through coil $i$ due to coil $j$ as

$$
\phi_{j-1}=. I_{i j} I_{j}
$$


and the total flux through each coil as

$$
\begin{aligned}
& \Phi_{i}=M_{i i} I_{i}+M_{i, j} I_{j} \\
& \Phi_{j}=M_{j i} I_{i}+M_{j j} I_{j}=M_{i, j} I_{i}+M_{j, j} I_{j}
\end{aligned}
$$

where use has been made of the symmetry relation $M_{i j}=M_{j i}$ derived in Appendix C. The constant flux requirement then gives

$$
\begin{aligned}
& \nabla_{i} \Phi_{i}=M_{i i} \nabla_{i} I_{i}+I_{i} \nabla_{i} M_{i i}+M_{i j} \nabla_{i} I_{j}+I_{j} \nabla_{i} M_{i j}=0 \\
& \nabla_{i} \Phi_{j}=M_{i j} \nabla_{i} I_{i}+I_{i} \nabla_{i} M_{i j}+M_{j j} \nabla_{i} I_{j}=0
\end{aligned}
$$

where the $M_{j, j}$ term is completely independent of $R_{i}$ and $Z_{i}$. The total energy can be expressed in terms of the fluxes as

$$
U_{t}=\frac{1}{2}\left(\Phi_{i} I_{i}+\Phi_{j} I_{j}\right)
$$

Taking the local gradient $\left(\nabla_{i}\right)$ of this expression and invoking the flux conservation requirement results in

$$
\nabla_{i} I_{t}=-\frac{1}{2} I_{i}^{2} \nabla_{i} M_{i i}-I_{i} I_{i} \nabla_{i} M_{i, j}
$$

The force on coil $i$ is, in turn, given by

$$
\mathbf{F}_{i}=-\left(\nabla_{i}\left(I_{t}\right)_{\phi}=\frac{1}{2} I_{i}^{2} \nabla_{i} M_{i i}+I_{i} I_{j} \nabla_{i} H_{i j}\right.
$$

where the subscript $\phi$ indicates that the energy gradient has been computed with respect to the constant flux requirement. This expression can now be generalized to an $N$ coil system, giving

$$
\mathbf{F}_{i}=I_{i}^{2} \nabla_{i}\left(\frac{U_{i-i}}{I_{i}^{2}}\right)+I_{i} \sum_{j=1, j \neq i}^{N} 2 I_{j} \nabla_{i}\left(\frac{U_{,}-i}{I_{i} I_{j}}\right)
$$

Note that in the previous expression, all of the energy terms are independent of the currents $I_{l}$ and $I_{j}$. Given this expression for the forces, it is only a matter of performing some straightforward algebra' to obtain the net force acting on a given coil.

\subsubsection{Force Between Two Distributed Currents}

The force on a current $J_{i}$ due to a current $J_{j}$ is computed from

$$
\begin{aligned}
\mathbf{F}_{\mathbf{j} \rightarrow \mathbf{i}} & =\mathbf{e}_{\mathbf{R}} \frac{1}{2} \frac{\partial}{\partial R_{1}} \int_{i} \mathbf{J}_{\mathbf{i}}(\mathbf{r}) \cdot \mathbf{A}_{\mathbf{j}}(\mathbf{r}) d \tau \\
& +\mathbf{e}_{\mathbf{Z}} \frac{1}{2} \frac{\partial}{\partial Z_{1}} \int_{i} \mathbf{J}_{\mathbf{i}}(\mathbf{r}) \cdot \mathbf{A}_{\mathbf{j}}(\mathbf{r}) d \tau
\end{aligned}
$$

\footnotetext{
'Stere Appendix D for the full set of derivations
} 
Since the derivatives do not involve variables of integration, it is possible to cvaluate this expression directly. The result found in equation D.4 is

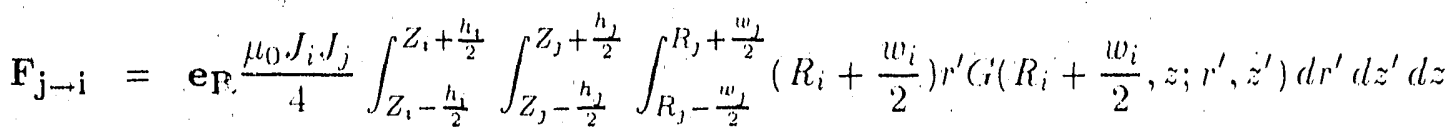

$$
\begin{aligned}
& \text { - } \mathbf{e}_{\mathbf{R}} \frac{\left.\mu_{1}\right) J_{i} J_{j}}{4} \int_{Z_{i}-\frac{h_{i}}{2}}^{Z_{1}+\frac{h_{1}}{2}} \int_{Z_{J}-\frac{h_{j}}{2}}^{Z_{j}+\frac{h_{j}}{2}} \int_{R_{j}-\frac{w_{j}}{2}}^{R_{j}+\frac{w_{j}}{2}}\left(R_{i}-\frac{w_{i}}{2}\right) r^{\prime} G\left(R_{i}-\frac{w_{i}}{2}, z ; r^{\prime}, z^{\prime}\right) d r^{\prime} d z^{\prime} d z \\
& +\mathrm{e}_{\mathrm{Z}} \frac{\mu_{0} \cdot J_{i} \cdot J_{j}}{4} \int_{R_{1}-\frac{\omega_{1}}{2}}^{R_{i}+\frac{\omega_{2}}{2}} \int_{Z_{3}-\frac{h_{j}}{2}}^{Z_{j}+\frac{h_{j}}{2}} \int_{R_{j}-\frac{w_{j}}{2}}^{R_{j}+\frac{w_{j}}{2}} r r^{\prime}\left(r^{\prime}\left(r, Z_{i}+\frac{h_{i}}{2} ; r^{\prime}, z^{\prime}\right) d r^{\prime} d z^{\prime} d r\right.
\end{aligned}
$$

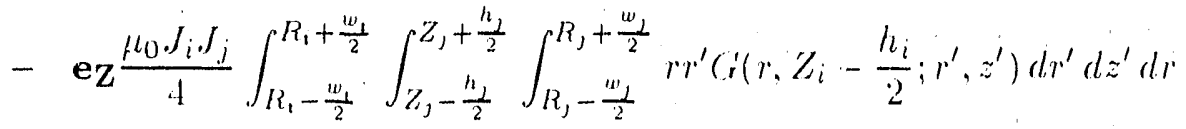

\subsubsection{Self Force of a Distributed Current}

Computation of a distributed current's self force is comparatively simple. Due to symmetry, the $\mathrm{e}_{\mathrm{Z}}$ component is zero. Taking the gradient of equation 5.8 results in equation D.5

$$
\begin{aligned}
& \mathbf{F}_{\mathrm{i}-\mathrm{i}}=\frac{\mu_{1} J_{i}^{2}}{2} \int_{Z_{1}-\frac{\omega_{i}}{2}}^{Z_{1}+\frac{\omega_{1}}{2}} \int_{Z_{1}-\frac{h_{i}}{2}}^{Z_{1}+\frac{h_{1}}{2}} \int_{R_{1}-\frac{w_{1}}{2}}^{R_{1}+\frac{w_{2}}{2}}\left(R_{i}+\frac{\omega_{i}}{2}\right) r^{\prime}\left(r^{\prime}\left(R_{i}+\frac{\omega_{i}}{2}, z ; r^{\prime}, z^{\prime}\right) d r^{\prime} d z^{\prime} d z\right. \\
& -\frac{\mu_{0} J_{i}^{\prime 2}}{2} \int_{Z_{1}-\frac{w_{1}}{2}}^{Z_{1}+\frac{w_{1}}{2}} \int_{Z_{1}-\frac{h_{1}}{2}}^{Z_{1}+\frac{h_{1}}{2}} \int_{R_{1}-\frac{w_{1}}{2}}^{R_{1}+\frac{w_{1}}{2}}\left(R_{i}-\frac{w_{i}}{2}\right) r^{\prime}\left(r^{\prime}\left(R_{i}-\frac{w_{i}}{2}, z ; r^{\prime}, z^{\prime}\right) d r^{\prime} d z^{\prime} d u\right.
\end{aligned}
$$

\subsubsection{Force Between 'Iwo Filamentary Currents}

The magnetic energy of two filamentary currents is given by equation 5.9. The corresponding expression for the force is given by equation D.1:

$$
\begin{aligned}
\mathrm{F}_{\mathrm{j} \rightarrow \mathrm{i}} & =\frac{\mu_{0} I_{i} I_{j}}{2} \frac{R_{i}+R_{j}}{A}\left\{K\left(k_{i}\right)\left[\frac{R_{i}}{R_{i}+R_{j}}\right]+\frac{E\left(k^{\prime}\right)}{1-k^{2}}\left[\frac{k^{2}}{2}-\frac{R_{i}}{R_{i}+R_{j}}\right]\right\} \mathrm{e}_{\mathbf{R}} \\
& +\frac{\mu_{0} I_{i} I_{j} Z_{i}-Z_{j}}{2}\left\{K\left(k_{i}\right)-E\left(k_{i}\right)\left(1+\frac{k^{2}}{2\left(1-k^{2}\right)}\right)\right\} \mathrm{e}_{\mathrm{Z}}
\end{aligned}
$$

\subsubsection{Self Force of a Filamentary Current}

As in the case of a distributed current, the ez component of the force is zero due to symmetry. The gradient of the energy can then be directly computed, resulting in equation D.2

$$
\mathbf{F}_{\mathrm{i} \rightarrow \mathrm{i}}=\frac{\mu_{0}}{2} l_{i}^{2}\left[\ln \left(\frac{R R_{1}}{a}\right)-1\right] \mathbf{e}_{\mathbf{R}}
$$




\subsection{Modelling the Coil-Coil Forces}

The three previous sections developed two possible sets of equations for use in computing the coil-coil interaction forces. This section is concerned with finding the best combination of these expressions to use in the final form of the force model The selection process will concentrate on the computational speed with which the forces can be computed and the overall accuracy of those forces. The first step in this process is to compare the results of the distributed and filamentary force equations.

Reconsider the two coil system shown in Figure 5-1. The first comparison consists of giving both coils the same major radius $\left(R_{0}=0.500 \mathrm{~m}\right)$ and the same height and width $(h=0.050 \mathrm{~m}$, $w=0.050 \mathrm{~m})$. Coil one is positioned at $Z_{1}=0.000$ and coil two is moved from $Z_{2}=-1.000 \mathrm{~m}$ to $Z_{2}=1.000 \mathrm{~m}$. In the region where coil two would intersect coil one $\left(-0.0 .50 \mathrm{~m} \leq Z_{2} \leq\right.$ $0.050 \mathrm{~m})$, the only point computed is $Z_{2}=Z_{1}=0$, which corresponds to the self-force of coil one.

Defining the force coefficient, $\mathbf{f}_{21}$, as

$$
\mathbf{f}_{21}=\mathbf{F}_{2-1} / \mu_{1} I_{1} I_{2}
$$

makes it possible to directly compare the results of the filamentary and distributed force models. The radial components of the force coefficient are compared in Figure 5-2, and the axial components of the force coefficient are compared in Figure 5-3. These two figures show that, for this case, the filamentary and distributed models are quite comparable.

An estimate of how well the distributed and filamentary current models agree can be obtained from the relative difference between them. This difference is defined as

$$
d_{r}=\frac{f_{f}-f_{d l}}{f_{f}}
$$

where $f_{f}$ is the force coefficient of the filamentary model and $f_{d}$ is the coefficient found with the distributed current model. The relative differences of the radial and axial components of the force coefficients are presented in Figures 5-4 and 5-5, respectively.

Figure 5-4 shows a sharp minimum at $Z_{2}=0$, illustrating the difference between the filamentary and distributed current self-forces. That the relative difference for this case is negative simply means that the self-force computed with the distributed current model is larger than the self-fore from the filamentary model. The relative difference for the remainder of the $z$ zalues 


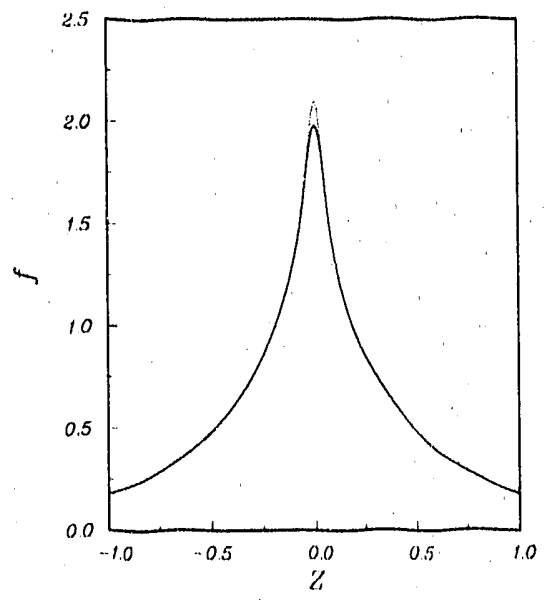

Figure 5-2: Comparison of the radial components of the force coefficient for an axially displaced coil. The solid line is the force coefficient found from the filamentary model. The dotted line represents the force coefficient computed from the distributed current model.

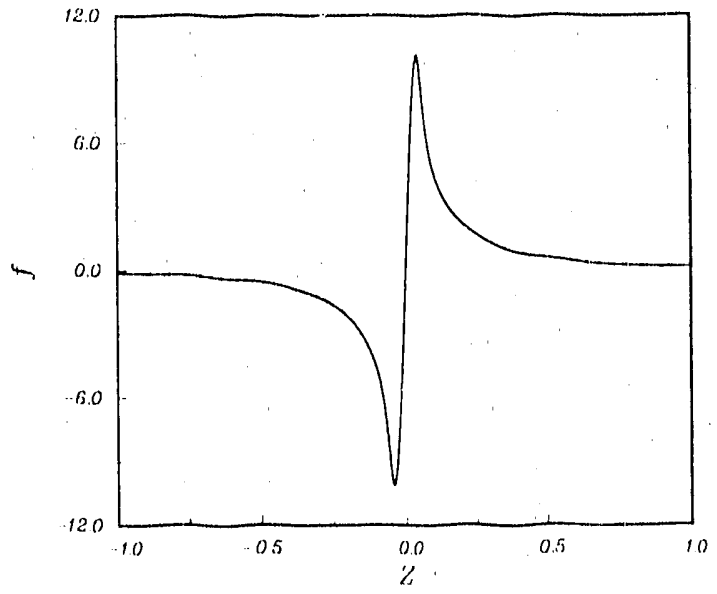

Figure 5-3: Comparison of the axial components of the foree coefficient for an axially displaced coil. The solid line in this figure is the force coefficient computed using the filamentary current model. The indistinguishable doted line is the coefficient found from the distributed current model. 


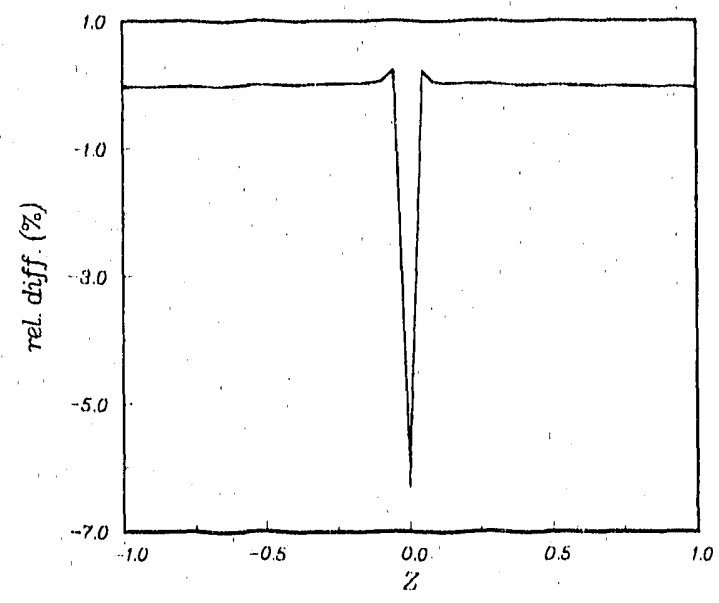

Figure 5-4: Relative difference between the radial force coefficients for the case of an axially displaced coil.

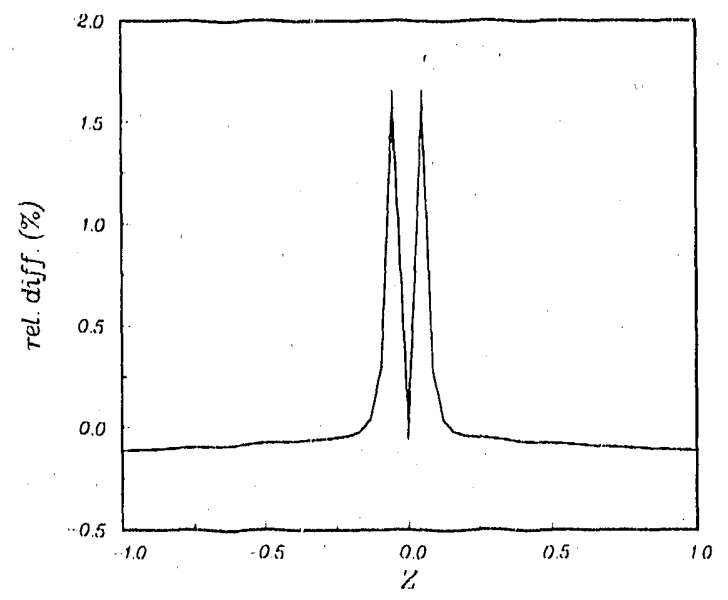

Figure 5-5: Rolative difference between the axial force coefficients for the case of an axially displaced coil. 


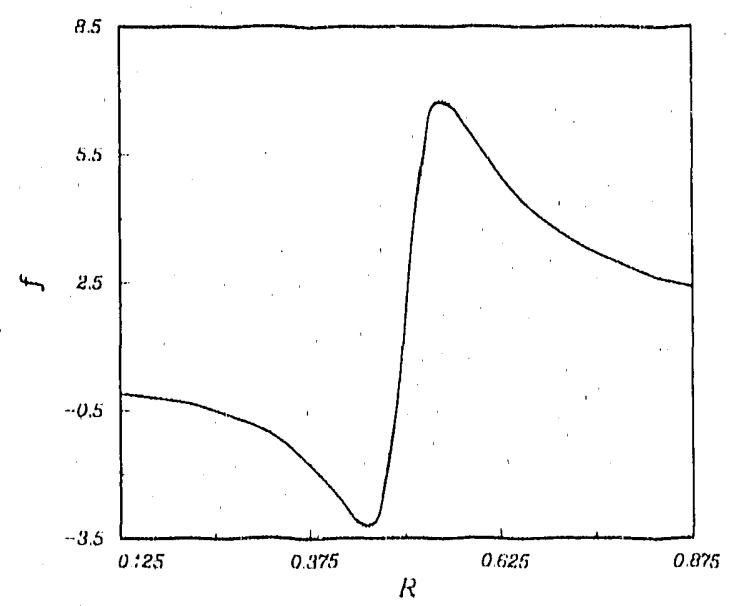

Figure 5-6: Comparison of the radial components of the force coefficient for the case of a coil with a varying radius. 'The solid line is the force coefficient found from the filamentary model. The dotted line represents the force coefficient. computed from the distributed current model.

is very nearly zero, indicating that, except for the self-force, there is practically no difference between the force coefficients found with the two models.

Examining Figure 5-5 shows similar behavior. Since the axial component of the self-force has been set to zero by symmetry, the relative difference at $z_{12}=0$ is zero. The remainder of the figure shows a very small relative difference except when the coils are very close together. In the region very near coil one, the magnetic field is decreasing very rapidly (recall that a dipole field falls off as $1 / R^{2}$ near the dipole). The filamentary current model is sampling this field at one specific point while the distributed current is sampling the field over its entire cross-section, leading to a smaller force coefficient for the distributed current model.

The next comparison to be made fixes $Z_{2}$ at a position of $0.050 \mathrm{~m}$ while the major radius varies in the range $0.25 R_{1} \leq R_{2} \leq 1.75 R_{1}$. The radial component of the force coefficient for this case is shown in Figure 5-6 and the axial component is shown in Figure 5-7. These two figures show that both the qualitative and quantitative behavior of the filamentary and distributed current force models agree quite well for this case:

The radial component of the relative difference for this case is shown in Figure 5-8. This figure shows two spikes near the position $R_{2}=R_{1}$. These spikes are again due to the overestimate 


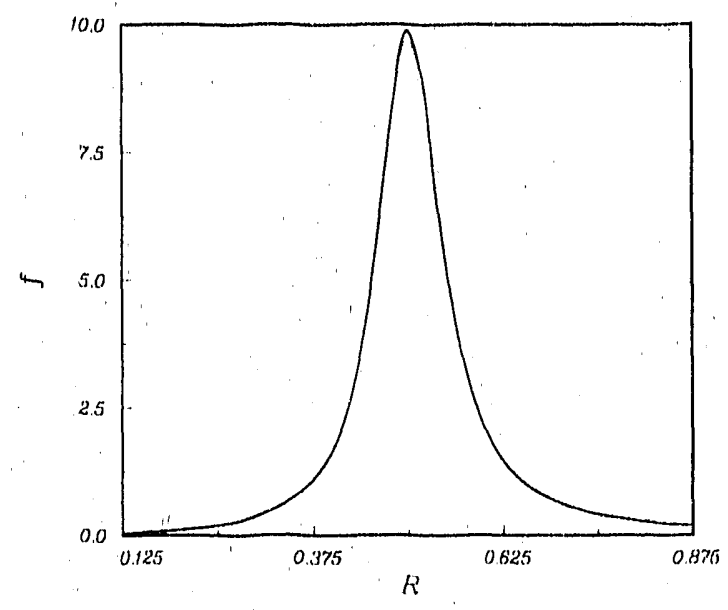

Figure 5-7: Comparison of the axial components of the force coefficient for the case is a coil with a varying radius. Tho solid line is the force coefficient found from the filamentary model. The dotted line represents the force coefficient computed from the distributed current model.

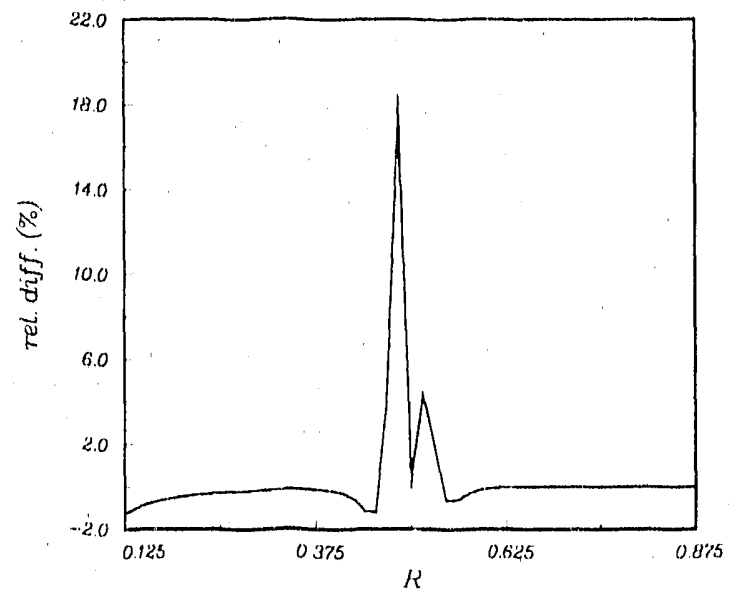

ligure 5-8: Radial component of the relative difference for a coil with a varying radius 


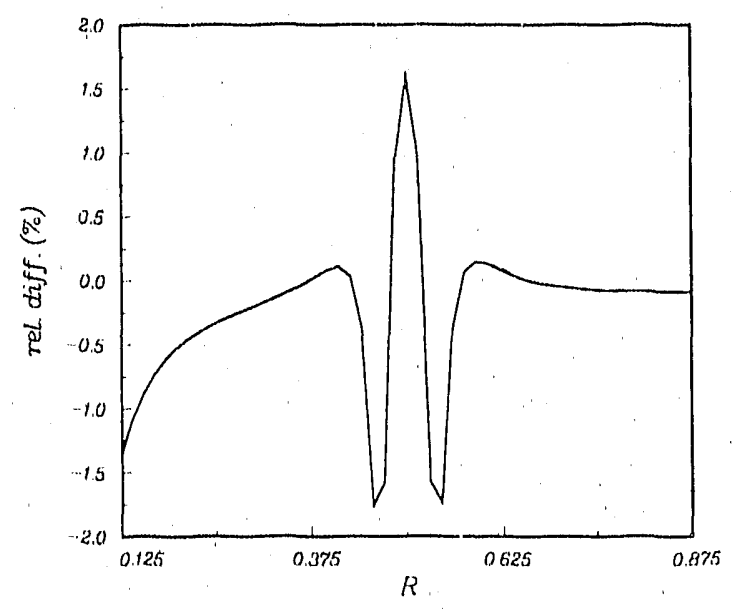

Figure 5-9: Axial component of the relative difference for a coil with a valying radius

of the force obtained from the filamentary model. The small value of the difference at $R_{2}=R_{1}$ is due to the negligible axial component of the magnetic field which occurs when coil two is positioned directly above coil one.

The axial component of the relative difference is shown in Figure 5-9. This figure again shows a large positive spike at $R_{2}=R_{1}$, indicating that the axial component of the force is being overestimated by the filamentary model. The smaller negative spikes on either side of $R_{2}=R_{1}$ show that the filamentary model underestimates the force in that region, again due to the fact that the filament is sampling only one point of the field while the distributed current is sampling the field over its cross-section:

The worst case results obtained from these two tests occur when the coils are very close together. In general, this situation occurs when there are a number of coils (wires) grouped together into a larger coil in which each of the wires carries the same current. The force interactions between the wires making up a single coil can, however, be ignored, since these forces are internal to the coil. Computation of the coil's self-force is performed by simply summing the self-forces of the wires making up the coil.

Since the two models produre nearly the same results, either of them would be suitable for estimating the coil-coil interaction forces. The next step is to estimate the amount of computation necessary to calculate the forces. The integrations required by the distributed current model were performed using the eight point Gaussian quadrature scheme presented in Appendix E. Thus, 
each integral required calculating the integrand 512 times. To calculate the force between two coils required evaluating the integrand 2048 times, and determining the self-force requires an additional 102.1 evaluations. Calculation of force between two coils using the filamentary model is done in only 2 functional evaluations, and the self-force is found from 1 additional evaluation.

For an $N$ wire system, the total number of coil-coil forces that must be calculated is given by $\left(N^{2}-N\right) / 2$. For the Alcator C-Mod coil set presented in the previous chapter, this number is 55,945 . Using the distributed current model to compute all of these forces would be computationally prohibitive (over 100 CPU minutes on a Cray 2). For this reason, the filamentary model is used to compute all of the wire-wire interaction forces. Since only $N$ self-forces have to bo computed, the user of the cPFC program is given the option to use either model, although the distributed current model is more accurate for this case.

\subsection{Plasma-Coil Interaction Forces}

In the previous sections, the forces due to coil-coil interactions were derived and calculated. This section is concerned with the calculation of the forces acting on the coils due to the plasma itself. The calculation itself uses a variation of the Green's function methodology presented in Chapter 2.

The force on a given coil due to the plasma can be found from

$$
\mathrm{F}_{\mathrm{p} \rightarrow \mathrm{i}}=\int_{V_{1}} \mathbf{J}_{\mathrm{i}} \times \mathrm{B}_{\mathrm{p}} d \tau
$$

where $J_{\mathrm{i}}$ is the current flowing in the $i$ th conductor, $\mathrm{B}_{\mathrm{P}}$ is the plasma magnetic field in the vacuum region, and $V_{i}$ is the volume of the ith conductor. Since the coils are considered 10 be far from the plasma (relative to the coil dimensions), $\mathbf{J}_{\mathbf{i}}$ can be represented as a filamentary current:

$$
\mathrm{J}_{\mathrm{i}}=l_{i} \phi\left(r-R_{i}\right) \delta\left(i-Z_{i}\right) \mathbf{e}_{\phi}
$$

where $I_{i}$ is the magnitude of the current flowing in coil $i$. By substituting equation 5.13 into equation 5.12 and invoking the toroidal symmetry assumption, $\mathrm{F}_{\mathrm{p}-\mathrm{i}}$ becomes:

$$
\mathrm{F}_{\mathrm{p} \rightarrow \mathrm{i}}=2 \pi I_{1}\left(\mathbf{e}_{\phi} \times \mathrm{B}_{\mathrm{p}}\left(R_{\iota}, Z_{i}\right)\right)
$$

As discussed in Chapter 2, the vacuum magnetic field due to the plasma can be represented as

$$
B_{p}=\Gamma_{0}+B_{i}
$$


where $\phi$ is the scalar magnetic potential of the plasma and $B_{1}$ is the field due to a single fllament assumed to carry all of the plasma current and located at the plasma magnetic axis. Substituting this into equation 5.14 results in

$$
\mathbf{F}_{\mathrm{p} \rightarrow \mathrm{i}}=2 \pi I_{i}\left(\mathbf{e}_{\phi} \times \nabla \phi+\mathbf{e}_{\phi} \times \mathbf{B}_{\mathbf{i}}\right)
$$

The last expression on the right hand side of equation 5.15 is simply the force between two filamentary coils. It is possible to replace that component of equation 5.15 with the equivalent expression formulated using the potential encrgy techniques described carlier in this chapter. Denoting this component as $\mathrm{F}_{\mathrm{pf} \rightarrow \mathrm{i}}$, equation 5.15 becomes

$$
\mathrm{F}_{\mathrm{p} \rightarrow \mathrm{i}}=2 \pi I_{i}\left(\mathbf{e}_{\phi} \times \nabla \phi\right)+\mathrm{F}_{\mathrm{p} \mathrm{f}^{\prime}-\mathrm{i}}
$$

where

$$
\begin{aligned}
\mathrm{F}_{\mathrm{pf} \rightarrow \mathrm{i}} & =\frac{\mu_{0} I_{i} I_{p}}{2} \frac{R_{i}+R_{m}}{A}\left\{K\left(k_{i}\right)\left[\frac{R_{i}}{R_{i}+R_{m}}\right]+\frac{E\left(k^{\prime}\right)}{1-k_{i}^{2}}\left[\frac{k^{2}}{2}-\frac{R_{i}}{R_{i}+R_{m}}\right]\right\} \mathrm{e}_{\mathrm{R}} \\
& +\frac{\mu_{0} I_{i} I_{p}}{2} \frac{Z_{i}-Z_{m}}{4}\left\{\Lambda(k)-E(k)\left(1+\frac{k^{2}}{2\left(1-k^{2}\right)}\right)\right\} \mathrm{e}_{\mathbf{Z}}
\end{aligned}
$$

In equation $5.17, R_{m}$ and $Z_{m}$ represent the coordinates of the magnetic axis of the plasma.

\section{1 The Scalar Potential}

With equations 5.16 and 5.17 , it is only necessary to compute $\nabla \phi$ to determine the force due to the plasma. In Chapter 2, $\phi$ was found on the plasma surface using Green's theorem. Green's theorem can also be used to compute $\phi$ in the space surrounding the plasma. Recall from Chapter 2

$$
\sigma \phi(\mathbf{r})+\int_{s_{p}}\left[\phi\left(\mathbf{r}^{\prime}\right)\left(\mathbf{e}_{11}^{\prime} \cdot \nabla^{\prime}\left(\hat{i}\left(\mathbf{r}, \mathbf{r}^{\prime}\right)\right)-C_{i}^{\prime}\left(\mathbf{r}, \mathbf{r}^{\prime}\right)\left(\mathbf{e}_{\mathbf{n}}^{\prime} \cdot \Gamma^{\prime} \phi\left(\mathbf{r}^{\prime}\right)\right)\right] d l s^{\prime \prime}=0\right.
$$

where $\mathbf{r}$ refers to the observation point, $\mathbf{r}^{\prime}$ refers to the plasma surface, and the value of $\sigma$ depends on the location of the observation point. For this case, the observation point is outside of the plasma surface, so $\sigma=1$.

In Chapter 2, use was made of the toroidal symmetry assumption to simplify equation 5.18 . As before, the primed variables denote the plasma surface, which is parameterized in terms of . Thus, equation 5.18 can be simplified to

$$
\phi(r, z)+\int_{0}^{2 \pi}\left[\phi\left(\mu^{\prime}\right) \frac{\partial\left(l^{\prime}\left(r^{\prime}, z, \mu^{\prime}\right)\right.}{\partial n^{\prime}}-\left(r^{\prime}\left(r, z, \mu^{\prime}\right) \frac{\partial \phi\left(\mu^{\prime}\right)}{\partial n^{\prime}}\right] R^{\prime} d \mu^{\prime}=0\right.
$$


where $(i$ is the reduced Green's function given by

$$
C^{\prime}=-\frac{k R^{\prime \prime}(k)}{2 \pi \sqrt{R R^{\prime}}}
$$

and $k$ is again defined as

$$
k^{2}=\frac{4 R R^{\prime}}{\left(R+R^{\prime}\right)^{2}+\left(Z-Z^{\prime}\right)^{2}}
$$

The majority of Chapter 2 was concerned with finding $\phi\left(\mu^{\prime}\right)$ and $\left.\left.R^{\prime}\right) \phi\left(\mu !^{\prime}\right) / i\right) n n^{\prime}$. With this information, it is a simple matter to evaluate equation 5.19 to find the scalar potential of the plasma at any point in space. It is also fairly easy to compute the gradient of equation 5.19 , which is the goal of the next section.

\subsubsection{Gradient of the Scalar Potential}

It is a reasonably straightforward process to compute the gradient of equation 5.19. Noting that the gradient involves only the spatial variables $r$ and $z$ and not the surface variables $R_{p}\left(\mu^{\prime}\right)$ and $Z_{p}\left(\mu^{\prime}\right)$ results in

$$
\Gamma \phi(r, z)=\int_{0}^{2 \pi}\left[\left(\nabla\left(r\left(r, z, \mu^{\prime}\right)\right) \frac{\partial \phi \phi\left(\mu^{\prime}\right)}{\partial n_{n^{\prime}}}-\phi\left(\mu^{\prime}\right)\left(\nabla \frac{\partial\left(c^{\prime}\left(r_{,}, z, \mu^{\prime}\right)\right.}{\partial n^{\prime}}\right)\right] R^{\prime} d \mu^{\prime}\right.
$$

The problem of computing $\nabla \phi$ has now been reduced to finding expressions for the gradients of $\left(i\right.$ and $\partial\left(i / \partial n^{\prime}\right.$, which is the subject of the next two subsections.

\section{The Gradient of $\mathrm{G}$}

Computing the gradient of the reduced Green's function is fairly simple. Starting with the e R component gives

$$
\begin{aligned}
\frac{\partial C i}{\partial R} & =-\frac{k K\left(k_{i}\right)}{2 \pi} \frac{\partial}{\partial R}\left(\frac{1}{\sqrt{R R_{p}}}\right)-\frac{1}{2 \pi \sqrt{R R_{p}}} \frac{\partial}{\partial k_{i}}\left(k_{i}\left(k_{i}\right)\right) \frac{\partial h_{i}}{\partial R} \\
& =\frac{k K\left(k_{i}\right)}{1 \pi R \sqrt{R R_{p}}}-\frac{1}{2 \pi \sqrt{R R_{p}}} \frac{E\left(k_{i}\right)}{1-k^{2}} \frac{\partial k_{i}}{\partial R}
\end{aligned}
$$

where use has been made of the identities in equation 2.33 .

Using the definition of $k$ gives

$$
\frac{\partial k}{\partial R}=h_{i}\left(\frac{1}{2 R}-\frac{R_{1}+R}{R^{2}}\right)
$$


where

$$
A^{2}=\left(R_{p}+R\right)^{2}+\left(Z_{p}-Z\right)^{2}
$$

The net result of this derivation is

$$
\frac{\partial C}{\partial R}=\frac{k}{4 \pi R \sqrt{R R_{p}}}\left[K(k)-\frac{E\left(k^{\prime}\right)}{1-k^{2}}\left(1-\frac{k^{2}}{2}\left(1+\frac{R_{p}}{R}\right)\right)\right]
$$

Determining the ez component is a little easicr, since the only dependence on $Z$ is in $k$. Noting that

$$
\frac{\partial k}{\partial Z}=\frac{k\left(Z_{p}-Z\right)}{A^{2}}
$$

results in

$$
\frac{\partial(i}{\partial Z}=\frac{k}{4 \pi R \sqrt{R R_{p}}}\left[\frac{E\left(k_{i}\right)}{1-k^{2}}\left(\frac{k^{2}}{2 R_{p}}\left(Z-Z_{p}\right)\right)\right]
$$

\section{The Gradient of $\partial\left(i / \partial n n^{\prime}\right.$}

The normal derivative of the reduced Green's function is given by

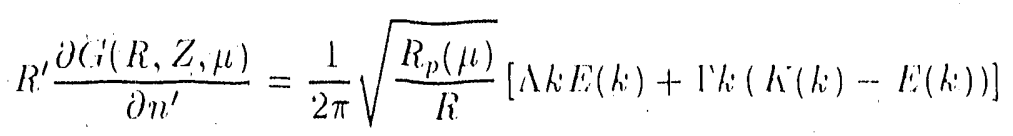

where

$$
\begin{aligned}
\Lambda & =\frac{Z_{p \mu}\left(R_{p}(\mu)-R\right)-R_{p \mu}\left(Z_{p}(\mu)-Z\right)}{\left(R_{\mu}(\mu)-R\right)^{2}+\left(Z_{\mu}(\mu)-Z\right)^{2}} \\
\Gamma & =\frac{Z_{p \mu}}{2 R_{p}(\mu)}
\end{aligned}
$$

In the above relations,

$$
\begin{aligned}
& Z_{p \mu}=\frac{\partial Z_{p}(\mu)}{\partial \mu} \\
& R_{p \mu}=\frac{\partial R_{p}(\mu)}{\partial \mu}
\end{aligned}
$$

Determining the gradient of the normal derivative of the reduced Green's function is more dificicult due to the complexity of equation 5.27. Propagating the $a / \partial R$ operator through equation 5.27 results in

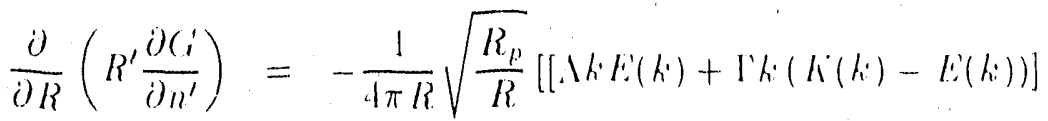

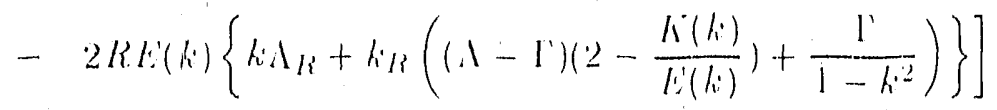


where the derivative of $A$ with respect to $R$ is given by

$$
\Lambda_{R}=\frac{Z_{p \mu}\left[\left(R_{p}-R\right)^{2}-\left(Z_{p}-Z\right)^{2}\right]-2 R_{p \mu}\left(Z_{p}-Z\right)\left(R_{p}-R\right)}{\left[\left(R_{p}-R\right)^{2}+\left(Z_{p}-Z\right)^{2}\right]^{2}}
$$

and $h_{R}$ is given by equation 5.23 .

The $e_{Z}$ component is found similarly, resulting in

$$
\frac{\partial}{\partial Z}\left(R^{\prime} \frac{\partial G}{\partial n^{\prime}}\right)=\frac{E(k)}{2 \pi} \sqrt{\frac{R_{p}}{R}}\left\{k \Lambda_{Z}+k_{z}\left((\Lambda-\Gamma)\left(2-\frac{K(k)}{E(k)}\right)+\frac{\Gamma}{1-k^{2}}\right)\right\}
$$

where

$$
\Lambda_{Z}=\frac{R_{p \mu}\left[\left(R_{p}-R\right)^{2}-\left(Z_{p}-Z\right)^{2}\right]+2 Z_{p \mu}\left(Z_{p}-Z\right)\left(R_{p}-R\right)}{\left[\left(R_{p}-R\right)^{2}+\left(Z_{p}-Z\right)^{2}\right]^{2}}
$$

and $k_{i} z$ is given by equation 5.25 .

\subsection{Plasma Force Equations}

The various equations for the plasma-coil force components can now be combined to determine the tysal force due to the plasma. The radial component of the plasma force on a coil is found from

$$
\begin{aligned}
\left(\mathrm{F}_{\mathrm{p} \rightarrow \mathrm{i}}\right)_{R} & =2 \pi l_{i} \frac{\partial O}{\partial Z}+\frac{\mu_{i} I_{i} I_{p}}{2} \frac{R_{i}+R_{m}}{1} \\
& \times\left\{h(k)\left[\frac{R_{i}}{R_{i}+R_{m}}\right]+\frac{E\left(k_{i}\right)}{1-k^{2}}\left[\frac{k^{2}}{2}-\frac{R_{i}}{R_{i}+R_{m}}\right]\right\}
\end{aligned}
$$

while the axial component is given by

$$
\begin{aligned}
\left(\mathrm{F}_{\mathrm{p}-\mathrm{i}}\right)_{Z} & =-2 \pi I_{i} \frac{\partial \partial_{0}}{\partial R} \\
& +\frac{\mu_{0} I_{i} I_{p}}{2} \frac{Z_{1}-Z_{m}}{A}\left\{h\left(h_{i}\right)-E\left(k_{i}\right)\left(1+\frac{k_{i}^{2}}{2\left(1-k^{2}\right)}\right)\right\}
\end{aligned}
$$

The derivatives of $D$ used in the preceeding equations correspond to the appropriate component of Equation 5.22 .

Combining the plasma-coil force expr ssions with those obtained for the coil-coil forces makes it possible to compute the total force acting on a coil. This, in turn, makes it possible to invoke force constraints on the coil currents in the same way that limits to the coil currents were invoked in Chapter 4. The details of implementing these constraints and the results are the subject of the next chapter. 


\section{Chapter 6}

\section{Nonlinear Constraints}

In Chapter 5, the expressions needed to compute the forces on the poloidal field coils were developed. This chapter is concerned with the force constraints and presents the formulation. used to determine the coil currents in the presence of these constraints. The resulting system is then tested. Since the methods used in this chapter to constrain the coil currents are identical to those used in Chapter 4, a demonstration of the system for a coil set with both current and force constraints is then given.

\subsection{The Source of the Constraints}

As discussed in the previous chapter, the coils experience a force given by $\mathbf{J} \times \mathbf{B}$. The engineering properties of the coil limit the amount of force it can carry.

Consider a single copper coil $1.0 \mathrm{~cm}$ wide in the radial direction, $1.0 \mathrm{~cm}$ in height, and with a major radius of $1.0 \mathrm{~m}$. For the purposes of this discussion, this coil will be treated as a $1.0 \mathrm{~cm}$ slice of an infinitely long pressure vessel. This approximation is made to simplify the derivation of the maximum forces in the radial direction. The maximum force the coil can carry in the radially outward direction is determined from

$$
S=\frac{p r}{1}
$$

where $p$ is the internal pressure acting on the coil, $;$ is the major radius of the coil, $t$ is the (radial) thickness of the coil, and $S$ is the tensile stress in the coil. To determine the maximum pressure this coil can carry, the tensile stress is replaced by the yield strength of the coil material. For annealed copper, the yiels. strength is 5000 psi. For the coil described above, the maximum 
internal pressure is $50 \mathrm{psi}$, corresponding to a maximum force of $10.83 \mathrm{kN}$ acring on the coil in the radially outward direction.

When a thin walled cylinder is subjected to a radially inward force the cylinder can fail by collapsing. The collapsing pressure is given by [1]

$$
p_{c}=K E\left(\frac{t}{2 r}\right)^{3}
$$

where $E$ is the Young's modulus of the coil material and $K$ is a numerical coefficient. Assuming that the coil is a section of an infinitely long cylinder allows $K$ to be approximated from

$$
r=\frac{2}{1-\mu^{2}}
$$

where $\mu$ is Poisson's ratio for the material. Poisson's ratio for copper is 0.355 and Young's modulus is $15.6 \cdot 10^{6} \mathrm{psi}$. These values result in a collapsing pressure of $35.169 \mathrm{psi}$, so that the maximum allowable force in the radially inward direction for this coil is $7.62 \mathrm{kN}$.

Because the radially inward and outward force limits may be different, the force constraints in the radial direction are written in the form

$$
\mathrm{F}_{\mathrm{Ri}} \leq \mathrm{F}_{\mathrm{R}} \leq \mathrm{F}_{\mathrm{Ro}}
$$

where $\mathbf{F}_{\mathbf{R}}$ is the vector of coil forces, $\mathbf{F}_{\mathbf{R i}}$ is the vector of radially inward force limits, and $\mathbf{F}_{\mathbf{R o}}$ corresponds to the radially outward force limits. Each of these vectors has a length of $J$, where $J$ is the total number of coils in the system.

The forces in the axial direction are assumed to be limited by the force that the coil supports can withstand. Denoting these axial force limits as $\mathbf{F}_{\mathrm{Zm}}$, the allowable force in the axial direction is specified by

$$
-\mathrm{F}_{\mathrm{Zm}} \leq \mathrm{F}_{\mathrm{Z}} \leq \mathrm{F}_{\mathrm{Zm}}
$$

where $\mathrm{F}_{\mathrm{Z}}$ is the vector of coil forces in the axial direction. Again, these vectors are all of length $J$.

\subsection{Formulation of the Constraint Equations}

The force expressions derived in Chapter 5 can be cast into two sets of linear equations with the form

$$
\begin{aligned}
& \mathbf{F}_{\mathbf{R}}=\mathbf{I}_{\mathrm{i}} \cdot\left(\mathbf{P}_{\mathrm{R}} \cdot \mathbf{i}+\mathbf{Q}_{\mathbf{R}}\right) \\
& \mathbf{F}_{\mathrm{Z}}=\mathrm{I}_{\mathrm{i}} \cdot\left(\mathbf{P}_{\mathrm{Z}} \cdot \mathrm{i}+\mathbf{Q}_{\mathrm{Z}}\right)
\end{aligned}
$$


where $\mathrm{F}_{\mathrm{R}}$ and $\mathrm{F}_{\mathrm{Z}}$ are vectors of length $J$ representing the radial and axial components of the coil forces. $\mathbf{P}_{\mathbf{R}}$ and $\mathrm{P}_{\mathbf{Z}}$ represent the intercoil forces in the radial and axial directions, as given by

$$
\mathbf{P}_{\mathbf{R}}=\left|\begin{array}{ccccc}
R_{11} & R_{12} & \cdots & \cdots & R_{1 n} \\
R_{21} & R_{22} & R_{23} & \cdots & R_{2 n} \\
\vdots & R_{32} & & & \vdots \\
\vdots & \vdots & & \vdots & \vdots \\
R_{n 1} & R_{n 2} & \cdots & \cdots & R_{n n}
\end{array}\right| \quad \mathbf{P}_{\mathbf{Z}}=\left|\begin{array}{ccccc}
0 & Z_{12} & \cdots & \cdots & Z_{1 n} \\
Z_{21} & 0 & Z_{23} & \cdots & Z_{2 n} \\
\vdots & Z_{32} & & & \vdots \\
\vdots & \vdots & & & \vdots \\
Z_{n 1} & Z_{n 2} & \cdots & \cdots & 0
\end{array}\right|
$$

where the $R_{i j}$ and $Z_{i j}$ are given by

$$
\begin{aligned}
& R_{i j}=\frac{\partial}{\partial R_{i}}\left(\frac{U_{j-i}}{I_{i} I_{j}}\right) \\
& Z_{i j}=\frac{\partial}{\partial Z_{i}}\left(\frac{U_{j-i}}{I_{i} I_{j}}\right)
\end{aligned}
$$

as found in Chapter 5. The $Q_{R}$ and $Q_{Z}$ vectors represent the radial and axial components of the sum of the forces due to any fixed coil currents and the plasma. Finally, the matrix of grouped currents $I_{i}$ is defined as

$$
\mathbf{I}_{\mathbf{i}}=\left|\begin{array}{ccccc}
i_{1} & 0 & \cdots & \cdots & 0 \\
0 & i_{2} & 0 & \cdots & 0 \\
\vdots & 0 & & & \vdots \\
\vdots & \vdots & & & \vdots \\
0 & 0 & \cdots & \cdots & i_{n}
\end{array}\right|
$$

Thus, the radial force on the $j$ th coil carrying the $n$th grouped current is given by

$$
\mathbf{F}_{\mathbf{R}_{, j}}=i_{n}\left(\sum_{k=1}^{N} \mathbf{P}_{\mathbf{R}_{, j, k} i_{k}}+\mathbf{Q}_{\mathbf{R}_{, l}}\right)
$$

where $N$ is the total number of grouped currents in the coil set.

Algebraically, the problem of minimizing the coil currents such that the force constraints are satisfied is expressed as

$$
\begin{array}{cl}
\text { Miumize } & \mathrm{i}^{T} \cdot \mathrm{M} \cdot \mathrm{i}-2 \mathrm{n}^{T} \cdot \mathrm{i}+\rho \\
\text { Subject io } & \mathrm{F}_{\mathrm{R} . \mathrm{i}} \leq \mathrm{F}_{\mathbf{R}}(\mathrm{i}) \leq \mathrm{F}_{\mathrm{Ro}} \\
& -\mathrm{F}_{\mathrm{Z}_{\mathrm{m}}} \leq \mathrm{F}_{\mathrm{Z}}(\mathbf{i}) \leq \mathrm{F}_{\mathrm{Zm}}
\end{array}
$$


The nonlinear nature of the force equations makes this a very difficult problem to solve. As in Chapter 4, it is assumed that a feasible point of Equation 6.4 is a satisfactory solution.

The method used to find a feasible point for the force constraint problem is identical to that presented in Sections 4.3 and 4.5 for the linearly constrained problem. Equation 6.4 is first replaced by the auxiliary function

$$
2 \gamma(\mathbf{i})=\mathbf{i}^{\mathrm{T}} \cdot \mathbf{M} \cdot \mathbf{i}-2 \mathbf{n}^{\mathrm{T}} \cdot \mathbf{i}+p+\alpha^{\mathrm{T}} \cdot \mathbf{q}+\beta^{\mathrm{T}} \cdot \mathbf{r}
$$

where $\alpha$ and $\beta$ are vectors of Lagrange multipliers and $\mathbf{q}$ and $\mathbf{r}$ are vectors corresponding to the constraint equations:

$$
\begin{aligned}
& q_{n}= \begin{cases}\mathbf{F}_{\mathbf{R}_{n}}-\mathbf{F}_{\mathbf{R i}_{n}} & \text { if } \mathbf{F}_{\mathbf{R}_{n}}<0 \\
\mathbf{F}_{\mathbf{R}_{n}}-\mathbf{F}_{\mathbf{R o} n} & \text { if } \mathbf{F}_{\mathbf{R}_{n}}>0\end{cases} \\
& r_{n}=\mathbf{F}_{\mathbf{Z}_{n}}-\mathbf{F}_{\mathbf{Z}_{n} n}
\end{aligned}
$$

Equation 6.5 can now be minimized. This is done by setting its derivative with respect to the grouped currents i to zero. Formally, this results in

$$
\frac{\partial \gamma(\mathbf{i})}{\partial \mathbf{i}}=\mathbf{M} \cdot \mathbf{i}-\mathbf{n}+\frac{\alpha^{\mathrm{T}}}{2} \cdot \frac{\partial \mathbf{q}(\mathbf{i})}{\partial \mathbf{i}}+\frac{\beta^{\mathrm{T}}}{2} \cdot \frac{\partial \mathbf{r}(\mathbf{i})}{\partial \mathbf{i}}=0
$$

To simplify matters, consider the equation for the $n$th current group with only one coil (coil $j$ ) being constrained:

$$
\frac{\partial \gamma(\mathbf{i})}{\partial i_{n}}=\sum_{k=0}^{N} M_{k, n} i_{k}-\mathbf{n}_{n}+\frac{\alpha}{2} \frac{\partial q_{j}(\mathbf{i})}{\partial i_{n}}+\frac{\beta}{2} \frac{\partial r_{j}(\mathbf{i})}{\partial i_{n}}=0
$$

Using the general form of the constraint equations given in Equation 6.3, the derivatives of $q$ and $r$ with respect to $i_{n}$ are given by

$$
\begin{aligned}
& \frac{\partial q_{j}(\mathbf{i})}{\partial i_{n}}=\frac{\partial i_{j}}{\partial i_{n}}\left(\sum_{k=1}^{N} \mathbf{P}_{\mathbf{R}_{, j, k^{i}} i_{k}}+\mathbf{Q}_{\mathbf{R}_{, j}}\right)+i_{j} \mathbf{P}_{\mathbf{R}_{, /, \prime}} \\
& \frac{\partial r^{\prime} j(\mathbf{i})}{\partial i_{n}}=\frac{\partial i_{j}}{\partial i_{n}}\left(\sum_{k=1}^{\mathrm{V}} \mathbf{P}_{\mathbf{Z}_{j, k} i_{k}}+\mathbf{Q}_{\mathbf{Z}_{\jmath}}\right)+i_{\jmath} \mathbf{P}_{\mathbf{Z}_{\jmath, n}}
\end{aligned}
$$

For the case of $N$ grouped currents with $h$ force constraints, Equation 6.8 has dimensions $\therefore$ by $N+2 K$. The remaining $2 K$ equations needed to complete the system are simply the force constraint equations given by Equations 6.6 and 6.7 . The resulting $N+2 k$ by $N+2 h$ system can then be solved using the algorithm presented in Section 4.5 . 
Computing the Jacobian of this system is a much more involved process, simply because of the nonlinearity of the force expressions. Additionally, the iterative nature of the algorithm requires that, after each iteration, the forces and the Jacobian be recomputed. The result is a computationally intensive atgolithm for finding a feasible point of the system.

\subsection{Verification, Validation, and Testing}

As with the linear constraint algorithm in Chapter 4, the force constraint algorithm is invoked only if the constraints are specified by the user. Verification of the program for this case requires ensuring that the results obtained for the cases presented in Section 3.3, Appendix A, and Section 4.6 are identical to the results obtained using the force constraint version of CPFC. These results were reproduced, indicating that the force constraint algorithm has no adverse effects on the results of the previous versions of the cPFC program.

Validation and testing the force constraint system requires running a number of cases with varying numbers of constraints and examining the results. Additionally, one case will be run using several values of the maximum force to illustrate the behavior of the system as the constraints become more restrictive. These tests will be run on the equilibrium and coil set shown in Figure 61. This coil set and equilibrium are identical to those used in Chapter 4. The wires making up the various coils are listed in Appendix B, and the OH1, EFCU, and EFCL coils are carrying fixed total currents of $1.4964 \mathrm{MA}, 3.0000 \mathrm{kA}$, and $-3.0000 \mathrm{kA}$, respectively. The equilibrium parameters are listed in Table 6.1 , and the unconstrained field and the equilibrium poloidal field are shown in Figure 6-2. The field error for the unconstrained casc is $1.408346 \%$. Finally, the coil forces and currents are listed in Table 6.2.

The first test case involves constraining the forces on the EF2L coil. For this case, the maximum force in the radially outward direction is set to $11.00 \mathrm{kN}$, and the maximum force in the axial direction is limited to $18.50 \mathrm{kN}$. Both of these constraints are violated by the unconstrained coil currents. The tangential component of the poloidal field produced by the coils and plasma is compared with that of the plasma itself in Figure 6-3. The ficld error for this case is $1.408669 \%$, and the coil forces and currents are listed in Table 6.3. The very small difference between the field error for this case and the unconstrained case is duc to the very slight amount by which the constraints are violated. Because the difference between this field eiror and the field error for the unconstrained case is so slight, there is no perceivable difference between the poloidal 


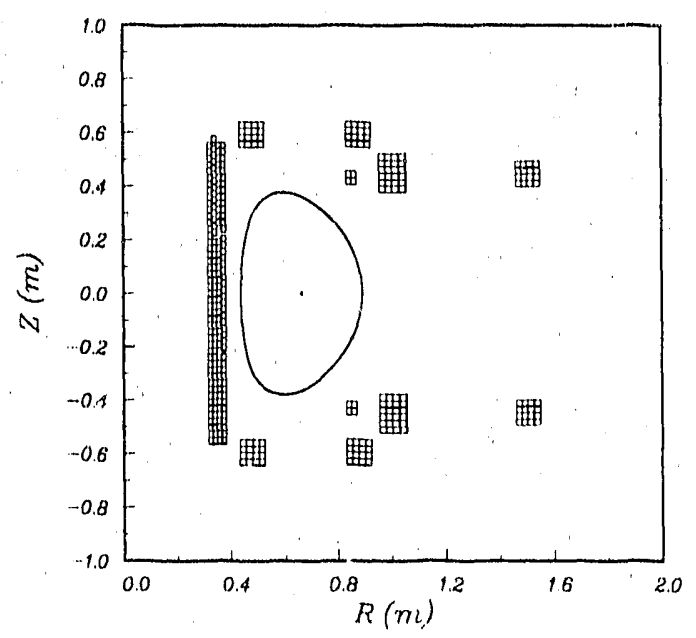

Figure 6-1: Alcator C-Mod coil set and equilibrium used for testing the force constraint algorithrn

Table 6.1: Equilibrium data for the Alcator (--Mod test case.

\begin{tabular}{|c|c|}
\hline \hline Parameter & Value \\
\hline $\mathrm{R}_{0}(\mathrm{~m})$ & 0.665 \\
$\mathrm{a}(\mathrm{m})$ & 0.225 \\
$\delta$ & 0.310 \\
$\kappa$ & 1.680 \\
$\mathrm{~B}_{0}(\mathrm{~T})$ & 8.500 \\
$\mathrm{I}_{p}(\mathrm{MA})$ & 2.500 \\
$\beta_{p}$ & 0.500 \\
$\beta_{t}(\%)$ & 0.017 \\
\hline
\end{tabular}




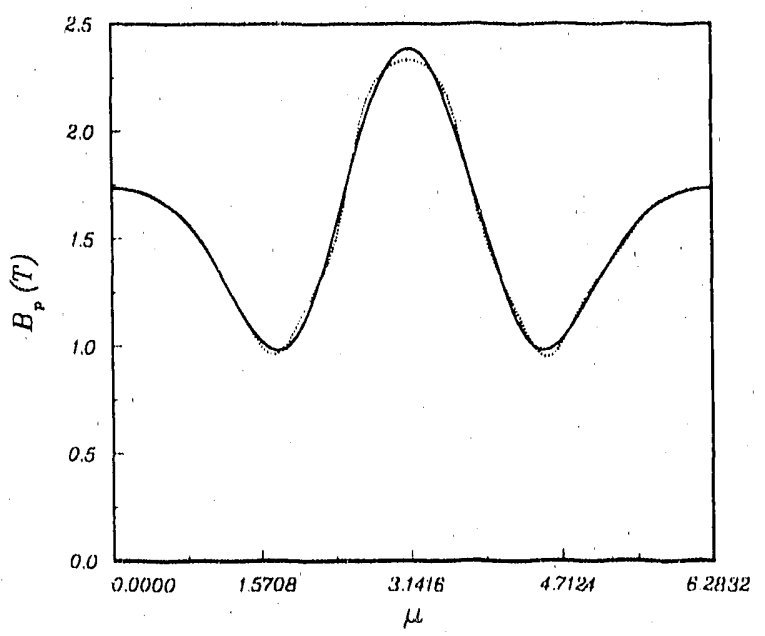

Figure 6-2: Poloidal fields for the unconstrained case. The solid line is the equilibrium field of the plasma. The dotted line represents the field due to the plasma and the PF coils.

Table 6.2: Unconstrained coil forces and currents.

\begin{tabular}{|l|r|r|r|}
\hline \hline $\begin{array}{c}\text { Coil } \\
\text { Label }\end{array}$ & $\begin{array}{c}\text { Radial } \\
\text { Force (MN) }\end{array}$ & $\begin{array}{c}\text { Axial } \\
\text { Force (MN) }\end{array}$ & $\begin{array}{c}\text { Current } \\
(\mathrm{kA})\end{array}$ \\
\hline OH1 & 10.509 & -0.014 & 11.600 \\
OH2U & 3.438 & -0.774 & 30.885 \\
OH2L & 3.398 & 0.9065 & 30.186 \\
EF1U & 0.496 & -2.413 & 51.274 \\
EF1L & 0.434 & 2.572 & 54.836 \\
EF2U & -0.456 & -0.161 & 6.618 \\
EF2L & 0.041 & -0.019 & -0.589 \\
EF3U & 0.6612 & 0.185 & -4.450 \\
EF3L & 0.6446 & -0.242 & -4.450 \\
EFCU & 0.150 & 0.082 & 7.500 \\
EFCL & -0.150 & 0.095 & -7.500 \\
EF4U & 2.715 & 0.669 & -54.720 \\
EF4L & 2.6668 & -0.726 & -54.720 \\
\hline \hline
\end{tabular}




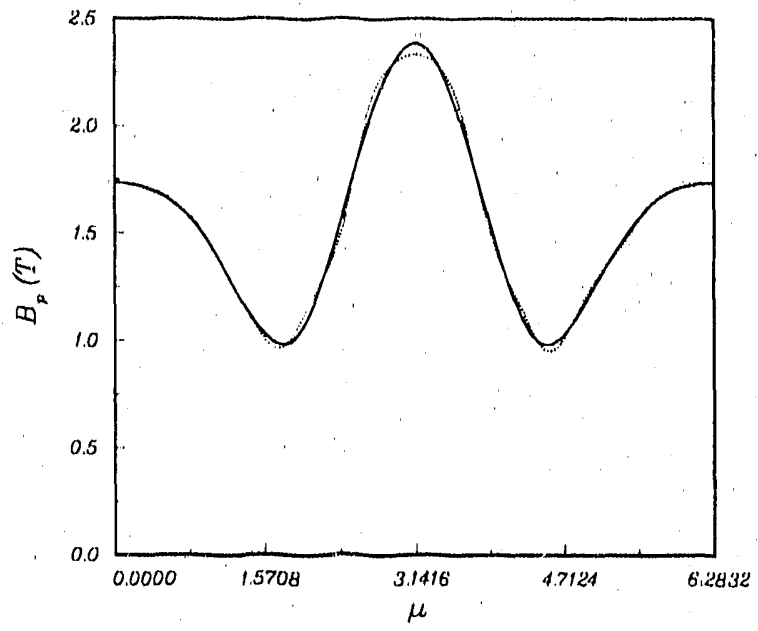

Figure 6-3: Poloidal fields for the case of one force constraint. The solid line is the equilib). rium field of the plasma, and the dotted line shows the field from the combination of the plasma and the PF coils.

Table 6.3: Coil forces and currents for the case of one coil with a force constraint:

\begin{tabular}{|l|r|r|r|}
\hline \hline $\begin{array}{c}\text { Coil } \\
\text { Label }\end{array}$ & $\begin{array}{c}\text { Radial } \\
\text { Force (MN) }\end{array}$ & $\begin{array}{c}\text { Axial } \\
\text { Force (MN) }\end{array}$ & $\begin{array}{c}\text { Current } \\
(\mathrm{kA})\end{array}$ \\
\hline OH1 & 10.364 & -0.015 & 11.600 \\
OH2U & 3.537 & -0.741 & 30.891 \\
OH2L & 3.496 & 0.877 & 30.211 \\
EF1U & 0.537 & -2.457 & 51.2944 \\
EF1L & 0.478 & 2.616 & 54.777 \\
EF2U & -0.456 & -0.162 & 6.573 \\
EF2L & 0.041 & -0.018 & -0.579 \\
EF3U & 0.638 & 0.180 & -4.3994 \\
EF3L & 0.642 & -0.2361 & -4.394 \\
EFCU & 1.152 & 0.082 & 7.500 \\
EFCL & -0.152 & 0.095 & -7.500 \\
EF4U & 2.722 & 0.665 & -54.796 \\
EF4L & 2.676 & -0.722 & -54.796 \\
\hline \hline
\end{tabular}


fields shown in Figure 6-3 for the constrained case and the fields shown in Figure 6-2 for the unconstrained casc.

The next case uses two active constraints. The force constraint on the EF2L coil is kept, and the EF2U coil is also constrained. The unconstrained radial force on the EF2U coil is directed inward, so these two constraints make it possible to test the full functionality of the algorithm. The maximum force in the radial direction is set to $0.45 \mathrm{MN}$, and the axial force is limited to $0.20 \mathrm{MN}$. Figure 6-4 compares the resulting poloidal field with that of the equilibrium alone. The coil forces and currents produced for this test are given in Table 6.4. Again, the very small amount by which the constraints are violated gives rise to a reasonably small ficld error, in this case $1.556010 \%$.

The third case tests the system for a constraint which is inactive. This is done by retaining the previous constraints and setting the maximum radial force on the EF1U coil to $5.00 \mathrm{MN}$ both inward and outward, and setting the maximum axial force to 5.00) MN. This constraint is clearly satisfied by the all three of the cases presented thus far. The equilibrium ficld is compared with the poloidal field generated by the plasma and coil set in Figure 6-5, and the coil forces and currents are listed in Table 6.5. The field error for this case is somewhat larger than for the previous cases at $1.663(122 \%$.

The final test for this case adds another inactive constraint to the three constraints already imposed on the system. For this test, the forces on the EF1L coil are limited to $5.000(0) \mathrm{MN}$ for both the inward and outward radial fore and $5.0000 \mathrm{MN}$ in the axial direction. These constraints are inactive for all of the tests performed so far. The coil forces and currents are listed in Table 6.6 and the tangential components of the equilibrium field and the poloidal field generated by the plasma and coils are illustrated in Figure 6-6. For this casc, the field error is $4.0071 .90 \%$.

The four cases present: ' in this section clearly show that the imposed force constraints are being satisfied. Also, the eflects of the algorithm discussed at the end of Chapter 4 are not as severe for the case of force constraints. This is due to the nonlinear nature of the force equations, which allow the coil currents to vary while keeping the forces on a given coil constant.

\subsection{Constraining Grouped Currents}

One issue which did not arise for the case of simple current limits is tha effect of a constraint when more than one coil carries the same current. This issue did not occur for the case of current 


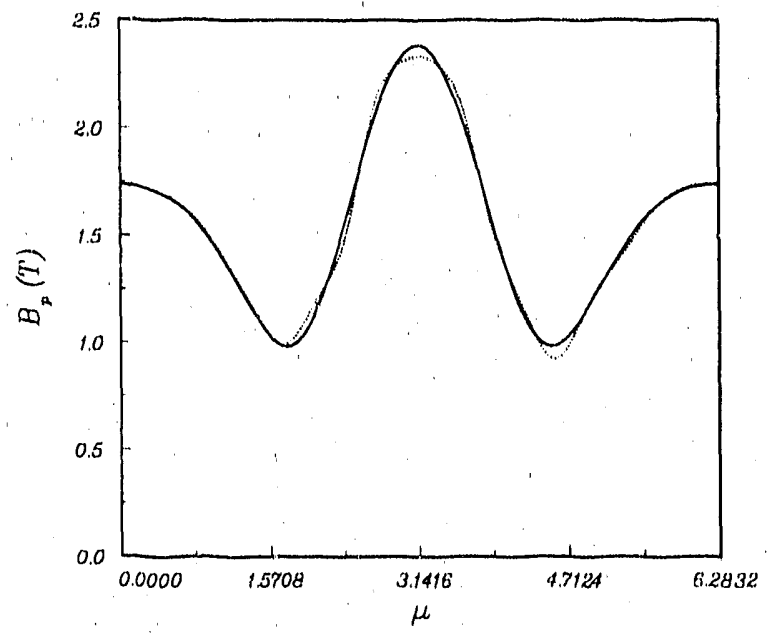

Figure 6-4: Poloidal fields for the case of two force constraints. The solid line represents the equilibrium field of the plasma, and the dotted line represents the field generated by the coils.

Table 6.4: Coil forces and currents for the case of two force constraints.

\begin{tabular}{|l|r|r|r|}
\hline \hline $\begin{array}{c}\text { Coil } \\
\text { Label }\end{array}$ & $\begin{array}{c}\text { Radial } \\
\text { Force (MN) }\end{array}$ & $\begin{array}{c}\text { Axial } \\
\text { Force (MN) }\end{array}$ & $\begin{array}{c}\text { Current } \\
(\mathrm{kA})\end{array}$ \\
\hline OH1 & 10.366 & -0.021 & 11.600 \\
OH2U & 3.555 & -0.778 & 31.406 \\
OH2L & 3.481 & 0.833 & 29.711 \\
EF1U & 0.510 & -2.422 & 50.034 \\
EF1L & 0.504 & 2.657 & 56.224 \\
EF2U & -0.450 & -0.162 & 6.514 \\
EF2L & 0.041 & -0.018 & -0.577 \\
EF3U & 0.619 & 0.175 & -4.247 \\
EF3L & 0.623 & -0.227 & -4.247 \\
EFCU & 0.151 & 0.083 & 7.500 \\
EFCL & -0.152 & 0.094 & -7.500 \\
EF4U & 2.750 & 0.673 & -55.114 \\
EF4L & 2.710 & -0.723 & -55.114 \\
\hline \hline
\end{tabular}




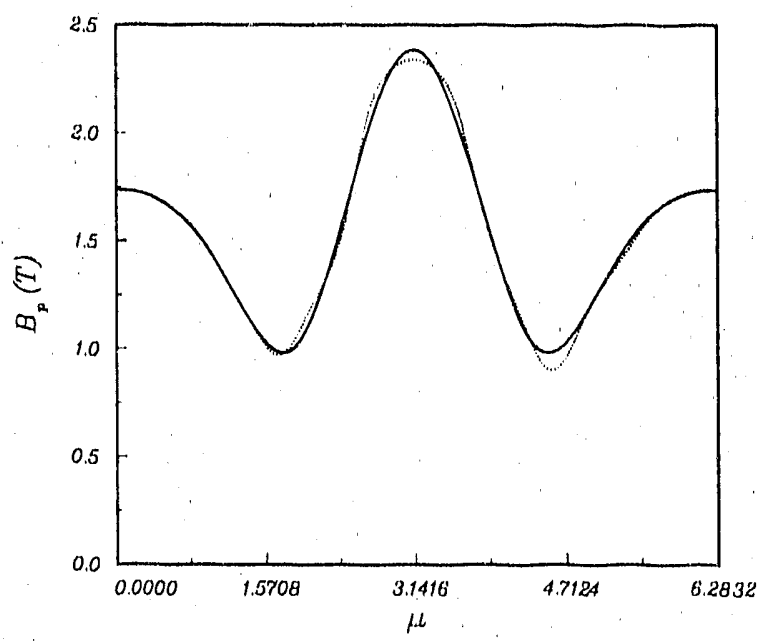

Figure 6-5: Poloidal fields for the case of three force constraints. The solid line is the field associated with the plasma equilibrium, and the dotted line is the sum of the plasma and PF coil fields.

Table 6.5: Coil forces and currents for the case of three force constraints.

\begin{tabular}{|l|rl|r|}
\hline $\begin{array}{c}\text { Coil } \\
\text { Label }\end{array}$ & \multicolumn{1}{c}{$\begin{array}{c}\text { Radial } \\
\text { Force }(\mathrm{MN})\end{array}$} & \multicolumn{1}{c|}{$\begin{array}{c}\text { Axial } \\
\text { Force }(\mathrm{MN})\end{array}$} & $\begin{array}{c}\text { Current } \\
(\mathrm{kA})\end{array}$ \\
\hline $\mathrm{OH} 1$ & 10.367 & -0.025 & 11.600 \\
OH2U & 3.534 & -0.743 & 30.831 \\
OH2L & 3.484 & 0.804 & 29.437 \\
EF1U & 0.538 & -2.456 & 51.264 \\
EF1L & 0.525 & 2.691 & 57.301 \\
EF2U & -0.450 & -0.162 & 6.502 \\
EF2L & 0.041 & -0.018 & -0.576 \\
EF3U & 0.612 & 0.173 & -4.198 \\
EF3L & 0.617 & -0.224 & -4.198 \\
EFCU & 0.151 & 0.082 & 7.500 \\
EFCL & -0.152 & 0.094 & -7.500 \\
EF4U & 2.759 & 0.675 & -55.179 \\
EF4L & 2.719 & -0.725 & -55.179 \\
\hline \hline
\end{tabular}




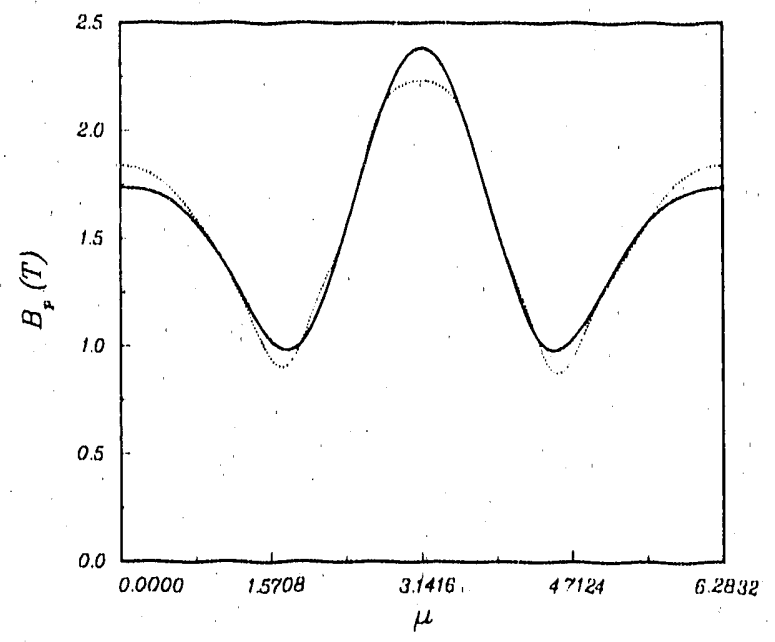

Figure 6-6: Poloidal fields for the case of four force constraints. The solid line shows the equilibrium field of the plasma, and the dotted line represents the sum of the plasma vacuum field and the fields of the PF coils.

Table 6.6: Coil forces and currents for the case of fond force constraints.

\begin{tabular}{|l|r|r|r|}
\hline $\begin{array}{c}\text { Coil } \\
\text { Label }\end{array}$ & $\begin{array}{c}\text { Radial } \\
\text { Force (MN) }\end{array}$ & $\begin{array}{c}\text { Axial } \\
\text { Force (MN) }\end{array}$ & $\begin{array}{c}\text { Current } \\
(\mathrm{kA})\end{array}$ \\
\hline OH1 & 9.908 & -0.021 & 11.600 \\
OH2U & 3.101 & -0.587 & 26.898 \\
OH2L & 3.103 & 0.692 & 26.628 \\
EF1U & 0.539 & -2.445 & 54.5653 \\
EF1L & 0.478 & 2.606 & 58.073 \\
EF2U & -0.448 & -0.162 & 6.218 \\
EF2L & 0.041 & -0.018 & -0.565 \\
EF3U & 0.396 & 0.098 & -2.505 \\
EF3L & 0.399 & -0.128 & -2.505 \\
EFCU & 0.153 & 0.080 & 7.500 \\
EFCL & -0.153 & 0.092 & $-7.500)$ \\
EF4U & 3.390 & 0.745 & $-(61.6655$ \\
EF4L & 3.3445 & -0.803 & -61.6655 \\
\hline \hline
\end{tabular}


constraints because the current carried by the grouped coils is treated as a single unique current by the global minimization algorithm. When grouped coils are subject to a force constraint, however, each coil must be treated independently. The nonlinearities of the force constraints can drastically affect the results. This will be demonstrated using three cases, all of which constrain the EF4U and EF4L coils. The maximum forces allowed on these coils are varied to obtain an idea of how the degree to which a constraint is violated affects the results.

For the unconstrained case presented in the previous section, the forces on the EF4U coil are 2.715 MN and 0.669 $\mathrm{MN}$ in the radial and axial directions, respectively. The radial force on the EF4L coil is $2.668 \mathrm{MN}$ and the axial force is $-0.726 \mathrm{MN}$. Setting the maximum value of the radial force to $2.700 \mathrm{MN}$ and the maximum axial force to $1.000 \mathrm{MN}$ gives the first set of test results. The coil currents and forces for this case are listed in Table 6.7. The resulting poloidal field is illustrated in Figure 6-7. The field error for this case is $5.303 \%$.

The second test imposes another constraint which is only violated by the EF4U coil, but now the violation is much worse. For this case, the maximum radial force on the colls is set to $2.675 \mathrm{MN}$. The poloidal fields for this case are shown in Figur 6-8, and the coil forces and currents are listed in Table 6.8. The field error for this is $14.044 \%$, much worse than for the previous case.

The final case for this section sets the maximum force in the radial direction for the EF4U and EF4L coils to $2.650 \mathrm{MN}$. The forces on both coils violate this constraint using the currents corresponding to the global minimum. The poloidal fields for this case are shown in Figure 6-9) and the coil forces and currents are shown in Table 6.9. The ficld error for this case is $16.068 \%$.

For this case, the field error grows steadily worse as the constraints grow more restrictive. This behavior is in part due to the sensitivity of the poloidal field to slight redistributions of the coil currents. The outboard coils (EF2U, EF2L, EFCU, EFCL, EF3U, EF3L, EF4U, and EF4L) are responsible for generating the rnajority of the poloidal field over the region of $\mu=-\pi / 2$ to $\mu=\pi / 2$. As the currents in these coils are adjusted to reduce the forces on the EF4U and EFAL coils, the ability of these coils to reproduce the equilibrium field over this region is greatly reduced. This problem may be avoided by adding more coils in this region or by allowing the EF3U, EF3L, EF4U, and EF4L to carry unique currents, giving the system the ability to adjust the current in each of these coils to reproduce the poloidal field at the plasma surface and satisfy the constraints simultaneousily. This assertion is born out by the results in Figure 6-10 and 


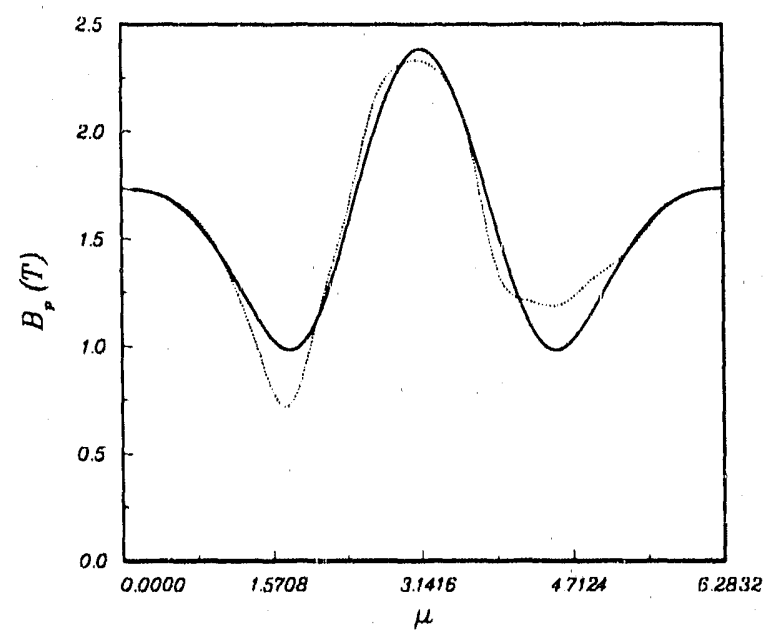

Figure 6-7: Poloidal fields for a force constraint on a set of grouped coils. The equilibrium field of the plasma is represented by the solid line. The dotted line shows the field generated by the PF system.

Table 6.7: Coil forces and currents for grouped coils subject to a force constraint.

\begin{tabular}{|l|r|r|r|}
\hline $\begin{array}{c}\text { Coil } \\
\text { Label }\end{array}$ & $\begin{array}{c}\text { Radial } \\
\text { Force (MN) }\end{array}$ & $\begin{array}{c}\text { Axial } \\
\text { Force (MN) }\end{array}$ & $\begin{array}{c}\text { Curr'nt } \\
(\mathrm{kA})\end{array}$ \\
\hline OH1 & 10.358 & 0.045 & 11.600 \\
OH2U & 3.313 & -0.413 & 26.027 \\
OH2L & 3.589 & 1.313 & 34.807 \\
EF1U & 0.725 & -2.813 & 65.199 \\
EF1L & 0.324 & 2.166 & 41.434 \\
EF2U & -0.268 & -0.091 & 3.712 \\
EF2L & -0.107 & -0.051 & 1.579 \\
EF3U & 0.6330 & 0.193 & -4.378 \\
EF3L & 0.639 & -0.227 & -4.378 \\
EFCU & 0.158 & 0.086 & 7.500 \\
EFCL & -0.146 & 0.092 & -7.500 \\
EF4U & 2.699 & 0.663 & -54.654 \\
EF4L & 2.668 & -0.720 & -54.65 .54 \\
\hline \hline
\end{tabular}




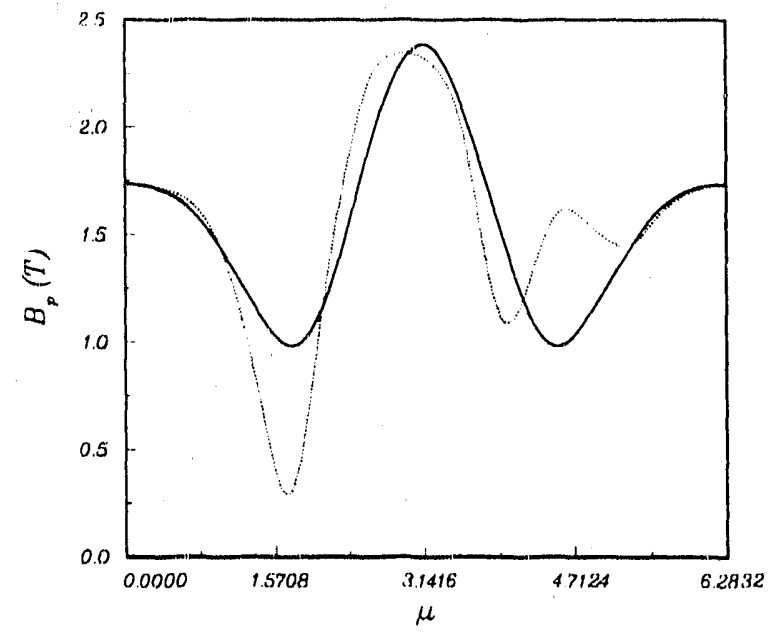

Figure 6-8: Poloidal fields for a constrained set of grouped coils. The equilibrium field of the plasma is shown by the solid line. 'The dott d line shows the sum of the plasma vacuum field with the field generated by the PF system.

Table 6.8: Coil forces and currents for grouped coils subject to force constraints.

\begin{tabular}{|l|r|r|r|}
\hline \hline $\begin{array}{c}\text { Coil } \\
\text { Label }\end{array}$ & $\begin{array}{c}\text { Radial } \\
\text { Force (MN) }\end{array}$ & \multicolumn{1}{|c|}{$\begin{array}{c}\text { Axial } \\
\text { Force (MN) }\end{array}$} & \multicolumn{1}{c|}{$\begin{array}{c}\text { Current } \\
(\mathrm{kA})\end{array}$} \\
\hline OH1 & 10.349 & 0.149 & 11.600 \\
OH2U & 2.632 & -0.030 & 17.609 \\
OH2L & 3.477 & 2.263 & 42.813 \\
EF1U & 1.094 & -3.121 & 89.332 \\
EF1L & 0.114 & 1.095 & 18.209 \\
EF2U & 0.102 & 0.033 & -1.325 \\
EF2L & -0.3333 & 0.17 .4 & 5.338 \\
EF3U & 0.604 & 0.209 & -4.253 \\
EF3L & 0.623 & -0.206 & -4.253 \\
EFCU & 0.168 & 0.094 & 7.500 \\
EFCL & -0.136 & 0.088 & -7.500 \\
EF4U & 2.674 & 0.664 & -54.540 \\
EF4L & 2.669 & -0.719 & -54.540 \\
\hline \hline
\end{tabular}




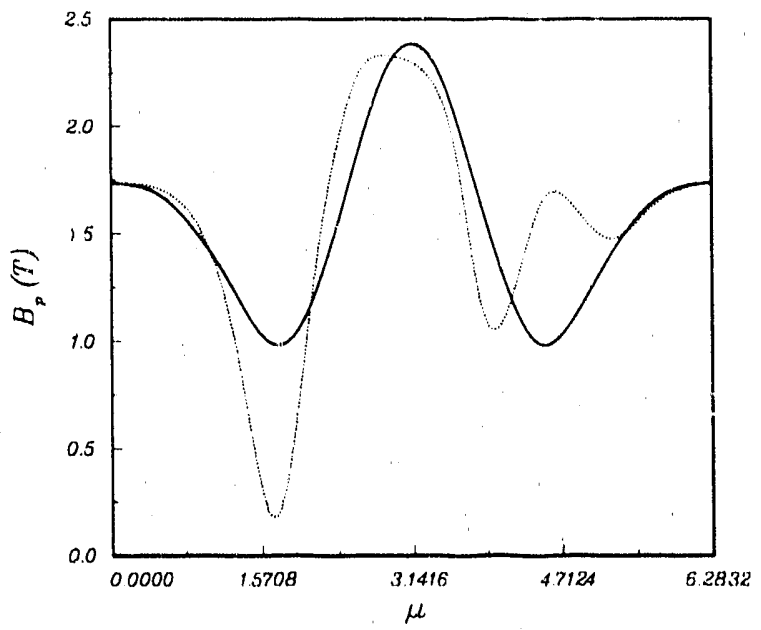

Figure 6-9: Poloidal fields for the case of a constrained sat of grouped coils. The solid line shows the equilibrium field of the plasma. The dotted line shows the field due to the PF system.

Table 6.9: Coil forces and currents for the case of constrained grouped coils.

\begin{tabular}{|l|r|r|r|}
\hline \hline $\begin{array}{c}\text { Coil } \\
\text { Label }\end{array}$ & $\begin{array}{c}\text { Radial } \\
\text { Force (MN) }\end{array}$ & $\begin{array}{c}\text { Axial } \\
\text { Force (MN) }\end{array}$ & $\begin{array}{c}\text { Current } \\
(\mathrm{kA})\end{array}$ \\
\hline OH1 & 10.245 & 0.173 & 11.600 \\
OH2U & 2.298 & 0.033 & 1.4 .843 \\
OH2L & 3.353 & 2.420 & 43.750 \\
EFiU & 1.133 & -3.111 & 96.172 \\
EF1L & 0.074 & 0.880 & 14.380 \\
EF2U & 0.332 & 0.108 & -4.235 \\
EF2L & -0.269 & 0.147 & 4.401 \\
EF3U & 0.551 & 0.208 & -3.9440 \\
EF3L & 0.571 & -0.197 & -3.9440 \\
EFCU & 0.171 & 0.100 & 7.500 \\
EFCL & -0.134 & 0.092 & -7.500 \\
EF4U & 2.649 & 0.664 & $-54.41: 3$ \\
EF4L & 2.650 & -0.719 & -54.413 \\
\hline \hline
\end{tabular}


Table 6.10. Here, the constraints imposed on the EF4 coils are those of the previous test, and the EF3U, EF3L, EF4U, and EF4L coils are allowed to carry unique currents. The field error for this case is $5.872 \%$.

\subsection{Combining Constraints}

Given that both the linear constraint and force constraint system are based on the same algorithm, it should be possible to have current and force constraints occurring simultaneously within the coil set. For this case, the problem of finding the coil currents is expressed as

$$
\begin{array}{cl}
\text { Minimize } & \mathbf{i}^{T} \cdot \mathbf{M} \cdot \mathbf{i}-2 \mathbf{n}^{T} \cdot \mathbf{i}+p \\
\text { Subject to } & \mathbf{F}_{\mathbf{R i}} \leq \mathbf{F}_{\mathbf{R}}(\mathbf{i}) \leq \mathbf{F}_{\mathbf{R o}} \\
& -\mathbf{F}_{\mathbf{Z} \mathbf{m}} \leq \mathbf{F}_{\mathbf{Z}}(\mathbf{i}) \leq \mathbf{F}_{\mathbf{Z} \mathbf{}} \\
& -\mathbf{b} \leq \mathbf{1} \leq \mathbf{b}
\end{array}
$$

where $l$ is the constrained subset of group currents and $\mathbf{b}$ is the corresponding set of maximum allowable currents.

The process of finding a feasible point proceeds as before, only now using three sets of constraint equations and three sets of Lagrange multipliers - two for the forces and one for currents. Since this process simply combines the analyses performed for the linear constraints and the force constraints, the details will be omitted here.

As a demonstration that the final version of the CPFC program can satisfy both current and force constraints, consider the coil set and equilibrium shown in Figure 6-1 once again. For this test, the current in the EF2U coil is limited to $6.500 \mathrm{kA}$ and the forces acting on the EF. $\mathrm{U}$ and EF3L coils are limited to $0.640 \mathrm{MN}$ in the radially outward direction and $1.00(0) \mathrm{MN}$ in the axial direction. The results of this test are shown in Figure 6-11 and the coil currents and forces are listed in Table 6.11. The field error for this test is $2.647 \%$.

Since the general problem combines both current and force constraints, the discussion at the end of Chapter 4 is applicable here, also. In Chapter 4 , it was found that a constraint which was inactive effectively locked the corresponding current to its initial value. Similar behavior was observed in the tests run in this chapter, but the nonlinear nature of the force constraints counteracted these effects, allowing the currents to vary will holding the force constant. These difficulties can be overcome by running CPFC first on the unconstrained case, examining the 


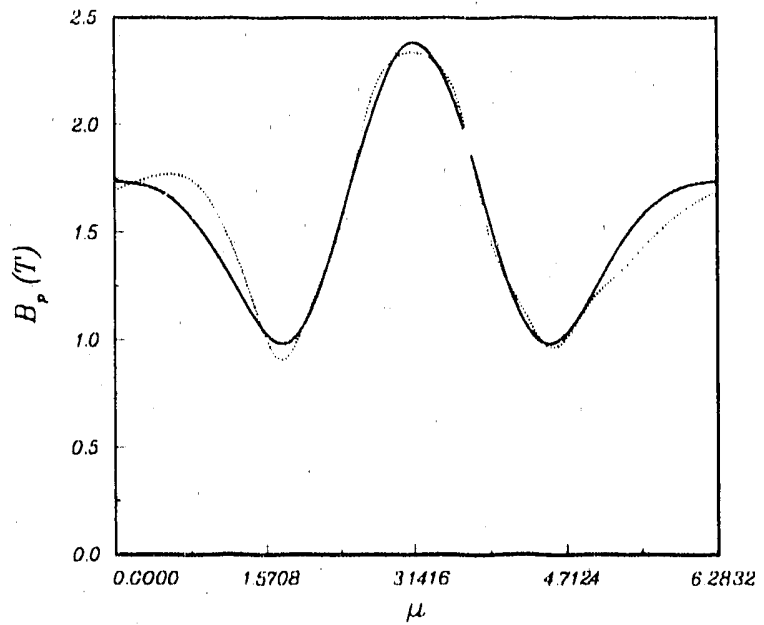

Figure 6-10: Poloidal fields for the previous case with the grouping requirement removed. The solid line shows the plasma equilibrium field. The dotted line shows the field due to the poloidal field coils.

Table 6.10: Coil forces and currents for the previous case with ungrouped coils.

\begin{tabular}{|c|c|c|c|}
\hline $\begin{array}{l}\text { Coil } \\
\text { Label }\end{array}$ & $\begin{array}{c}\text { Radial } \\
\text { Force }(\mathrm{MN})\end{array}$ & $\begin{array}{c}\text { Axial } \\
\text { Force (MN) }\end{array}$ & $\begin{array}{c}\text { Current } \\
(\mathrm{kA})\end{array}$ \\
\hline $\mathrm{OH} 1$ & 10.386 & -0.006 & 11.600 \\
\hline $\mathrm{DH} 2 \mathrm{U}$ & 3.493 & -0.593 & 29.355 \\
\hline $\mathrm{O} .42 \mathrm{~L}$ & 3.5663 & $0.9+40$ & 31.027 \\
\hline EF1: & 0.390 & -2.756 & 59.3330 \\
\hline $\mathrm{EF} 1 \mathrm{~L}$ & $0.5: 37$ & 2.527 & $5: 2.207$ \\
\hline $\mathrm{EF} 2 \mathrm{U}$ & 0.051 & 0.017 & $-(0.68 .5$ \\
\hline EF2L & 0.122 & -0.070 & $-1.99 \%$ \\
\hline EF3U & 0.802 & 0.3321 & -5.758 \\
\hline EF3L & 0.302 & $-(0.1 \cdot 20$ & -2.2666 \\
\hline EFCU & 0.170 & 0.106 & $T .500$ \\
\hline EFCL & -0.131 & 0.0995 & $-7.500)$ \\
\hline EF4U & 2.650 & $(0.997$ & -57.265 \\
\hline EF4L & 2.212 & -0.391 & -46.615 \\
\hline
\end{tabular}




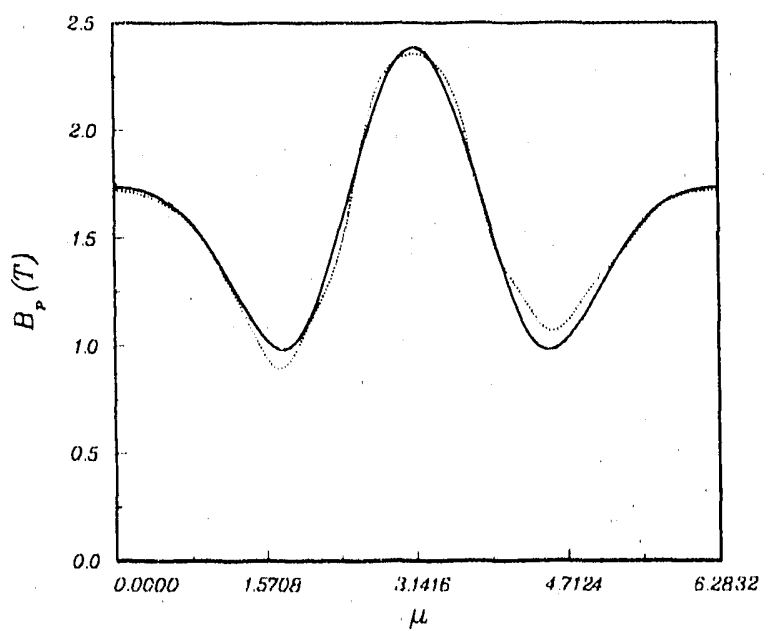

Figure 6-11: Poloidal fields for the general case of current and force constraints. The solid line represents the equilibrium field of the plasma. The dotted line shows the field generated by the PF system.

Table 6.11: Coil forces and currents for the general case of current and force constraints.

\begin{tabular}{|c|c|c|c|}
\hline $\begin{array}{c}\text { Coil } \\
\text { Label }\end{array}$ & $\begin{array}{c}\text { Radial } \\
\text { Force }(\mathrm{MN})\end{array}$ & $\begin{array}{c}\text { Axial } \\
\text { Force }(\mathrm{MN})\end{array}$ & $\begin{array}{c}\text { Current } \\
(\mathrm{kA})\end{array}$ \\
\hline $\mathrm{OH} 1$ & 10.482 & 0.046 & 11.600 \\
\hline $\mathrm{OH} 2 \mathrm{U}$ & 3.739 & -0.722 & 32.075 \\
\hline $\mathrm{OH} 2 \mathrm{~L}$ & 3.511 & 1.008 & 31.207 \\
\hline $\mathrm{EF} 1 \mathrm{U}$ & 0.564 & -2.576 & $53.3 .29 \cdot 2$ \\
\hline EF1L & 0.448 & 2.472 & $5(1.29)^{\circ}$ \\
\hline EF2U & -0.150 & -0.161 & $(j .50)$ \\
\hline EF2L & 0.003 & -0.001 & -0.0 .1 .4 \\
\hline EF3U & 0.6339 & 0.182 & -1.134 \\
\hline EF3L & 0.6 .10 & $-(0.239$ &.- .1 .138 \\
\hline $\mathrm{EFCU}$ & 0.152 & 0.082 & $7.500)$ \\
\hline EFCL & -0.149 & $0 .(095$ & -7.500 \\
\hline $\mathrm{EF} 4 \mathrm{U}$ & 2.665 & 0.654 & -5.4 .0336 \\
\hline EF4L & 2.6099 & -0.722 & -5.1 .0336 \\
\hline
\end{tabular}


results, and setting the constraints appropriately. By adding only one constraint at a time, it is possible to find a set of coil currents which satisfies all of the constraints and adequately supports the equilibrium.

The final topic for discussion is the execution time of the force constraint algorithm. Consider first the last test case of Section 6.3. This test imposed two active and two inactive force constraints on the coil currents. The CPU time necessary to run this case on a Cray-2 was 18.97 seconds. Of this, the force constraint algorithm required $1.025 \mathrm{CPU}$ seconds to determine the coil currents, and the time needed to generate the various quantities for computing the forces was 7.555 seconds. The unconstrained case required only $11.07 \mathrm{CPU}$ seconds to run, of which $0.685 \mathrm{CPU}$ seconds were devoted to finding the coil currents. These results show that the force constraint algorithm increases the total running time by approximately two-thirds. 


\section{Chapter 7}

\section{Conclusions and Recommendations}

As discussed in Chapter 1, this thesis has described the development of a design tool for the poloidal field magnet system of a tokamak. This was done by modifying an existing program to:

1. Better model realistic plasma and coil configurations,

2. Find a set of coil currents which support a specified equilibrium such that the maximum currents in the coils are not exceeded,

3. Find a set of coil currents to support an equilibrium such that the maximum allowable forces on the coils are not exceeded.

All of these goals were achieved with satisfactory results. There is, however, a substantial amourt of work which can be done to increase the usefulness of the cPFC program even further.

This work falls into two calegories. First, there is additional work needed to further verify the results of the cPFC prograrn. This requires benchmarking the CPFC program against other programs. Since the other available programs solve the free-boundary Grad-Shafranov equation, such a benchmark requires comparing the combined results of the ePFC program and Haney's fast equilibrium solver with those of the othrr programs. Another, potentially better, benchmark would involve using the CPFC program to reconstruct the coil currents from an actual experiment.

Additional work to the CPFC program itself includes, but is not limited to, the following:

- Implement an automated algorithm which determines an optimal set of coil currents, 
- Modify the program to automatically find the coil currents at a number of times during the ohmic discharge,

- Estimate the net force acting on the plasma,

- Add a separate program to calculate the intercoil forces, thereby reducing the execution time for $\mathrm{CPF}$ on finalized designs.

These additions to the progr um would further enhance its use as a design tool. 


\section{Appendix A}

\section{Verification of Asymmetric Program}

In Chapter 3 the changes needed to transform the original cPFC program into a form which would deal with asymmetric plasmas and coil sets were presented. The primary test of the new version of the program is to guarantee that it produce results which are identical to those obtained using the up-down symmetric version of CPFC for systems which are up-down symmetric. The data for one up-down symmetric case were presented in Chapter 3. In this appendix, data for two more cases will be presented. These three test cases provide sufficient proof that the asymmetric version exactly reproduces the results obtained with the symmetric version for symmetric cases.

\section{A.1 Case 1: A Conventional Tokamak}

In this section, the data for the symmetric and asymmetric versions of the cPFC program are compared for the case of the conventional tokamak coil set and plasma illustrated in Figure A-1. This equilibrium is characterized by the parameters in Table A.1.

The coil data for the case of the three coils being grouped up-down is given in Table A.2. The data for the coils being allowed to carry unique currents is presented in Table A.3. As expected for symmetric systems, the coil currents for the grouped and ungrouped cases are identical. The field error for this system is very small, only $0.75 \%$, indicating that the coils can very accurately produce the poloidal field required to maintain this equilibrium. Finally, the poloidal field data is shown in Figure A-2. The dotted line (indicating the field produced by the coils) is marginally displaced from the solid line representing the plasma's poloidal field. 


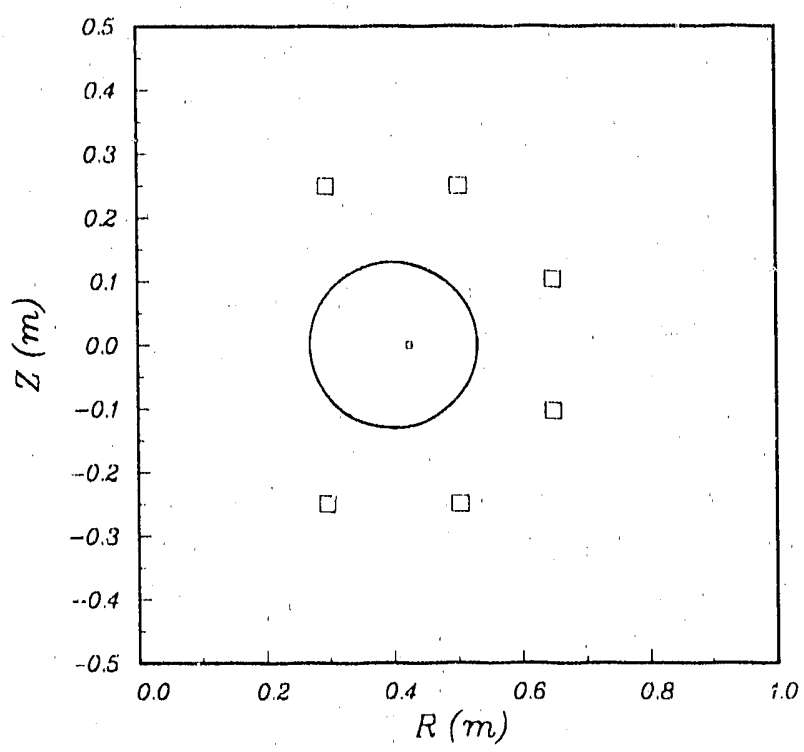

Figure A-1: A conventional tokamak coil set and circular plasma

Table A.1: Equilibrium data for the conventional tokamak test case.

\begin{tabular}{|c|c|}
\hline \hline Parameter & Value \\
\hline $\mathrm{R}_{0}(\mathrm{~m})$ & 0.400 \\
$\mathrm{a}(\mathrm{m})$ & 0.130 \\
$\delta$ & 0.000 \\
$\kappa$ & 1.000 \\
$\mathrm{~B}_{0}(\mathrm{~T})$ & 0.700 \\
$\mathrm{I}_{p}(\mathrm{kA})$ & 10.000 \\
$\beta_{p}$ & 1.000 \\
$\beta_{t}(\%)$ & 0.049 \\
\hline \hline
\end{tabular}


Table A.2: Data for coil groups EF1 (T) and EF1 (B), EF2 (T) and EF2 (B), and EF3 (T) and EF3 (B) carrying symmetric currents.

\begin{tabular}{|c|c|c|c|c|c|}
\hline & Symmetric & Asymmetric & & Symmetric & Asymmetric \\
\hline Coil & \multicolumn{2}{|c|}{ Current (kA) } & Coil & \multicolumn{2}{|c|}{ Current (kA) } \\
\hline $\mathrm{EF1}(\mathrm{T})$ & -1.951 & -1.951 & EF1 (B) & -1.951 & -1.951 \\
\hline $\mathrm{EF} 2(\mathrm{~T})$ & -1.724 & -1.724 & EF2 (B) & -1.724 & -1.724 \\
\hline \multirow[t]{2}{*}{$\mathrm{EF3}(\mathrm{T})$} & -1.432 & -1.432 & EF3 (B) & -1.432 & -1.432 \\
\hline & \multicolumn{2}{|c|}{ Error (\%) } & & & \\
\hline Field Error & 0.75265 & 0.75265 & & & \\
\hline
\end{tabular}

Table A.3: Data for coils EFL (T), EF1 (B), EF2 (T), EF2 (B), EF3 (T), and EF3 (B) carrying unique currents.

\begin{tabular}{|c|c|c|c|c|c|}
\hline & Symmetric & Asymmetrir & & Symmetric & Asymmetric \\
\hline Coil & \multicolumn{2}{|c|}{ Current (kA) } & Coil & \multicolumn{2}{|c|}{ Current (kA) } \\
\hline EF1 $(\mathrm{T})$ & -1.951 & -1.951 & EF1 (B) & -1.951 & -1.951 \\
\hline $\mathrm{EF} 2(\mathrm{~T})$ & -1.724 & -1.724 & EF2 (B) & -1.724 & -1.724 \\
\hline EF3 $(T)$ & -1.432 & -1.432 & EF3 (B) & -1.432 & -1.4332 \\
\hline & \multicolumn{2}{|c|}{ Error $(\%)$} & & & \\
\hline l'ield Error & 0.75265 & 0.75265 & & & \\
\hline
\end{tabular}




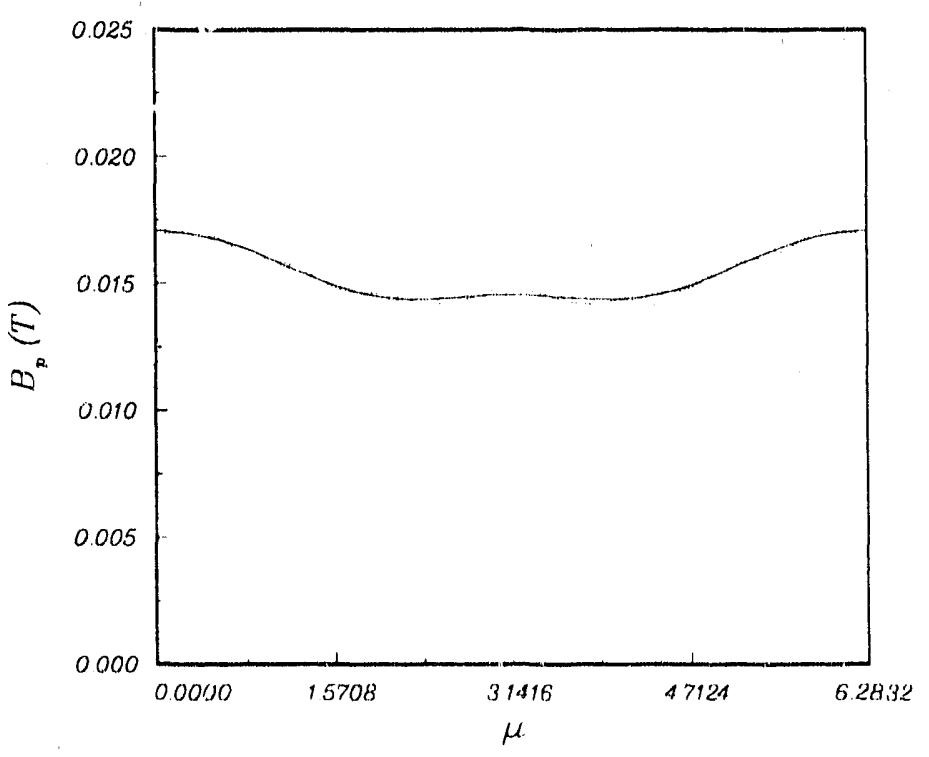

Figure A-2: Poloidal field comparison for the conventional tokamak case. The solid line is the poloidal field due to the plasma at the plasma surface. The dotted line (barely visible) is the field produced by the coils. 


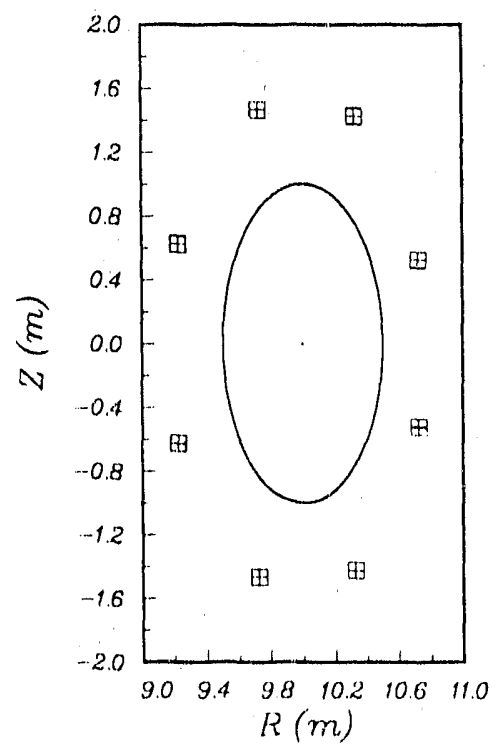

Figure A-3: Highly elongated tokamak plasma and coil set.

\section{A.2 Case 2: An Elongated Tokamak}

In this section, the data from the symmetric and asymmetric versions of the $\mathrm{CPFC}$ program are compared for the case of the highly elongated tokamak shown in Figure A-3. The plasma shown in Figure A-3 is characterized by the equilibrium data in Table A.4.

This test case is slightly different from the other two in that each coil is now made up of four unique wires. Each wire in an individual coil is constrained to carry the same current (as discussed in Chapter 2). For this case, then, the two tests consist of coils which are either grouped up-down or independent, while the wires comprising each coil carry the same current. The data presented here list the total current in each coil.

The data for the case where the coils are grouped up-down symmetrically are presented in Table A.5, and the data for the ungrouped case are given in Table A.6. Finally, the poloidal field generated by the combination of these coil currents and the plasma current is compared with the plasma's equilibrium poloidal field in Figure A-4. The difference between the two is quite apparent, clearly illustrating the $10 \%$ field error obtained for this system. This large error is due to the small number of coils in the system and indicates that this coil set is not capable of supporting the specified equilibrium. 
Table A.t: Equilibrium data for the highly elongated tokamak.

\begin{tabular}{|c|c|}
\hline Parameter & Value \\
\hline $\mathrm{R}_{0}(\mathrm{~m})$ & 10.000 \\
$\mathrm{a}(\mathrm{m})$ & 0.500 \\
$\delta$ & 0.000 \\
$\kappa$ & 2.000 \\
$\mathrm{~B}_{0}(\mathrm{~T})$ & 3.140 \\
$\mathrm{I}_{p}(\mathrm{MA})$ & 1.000 \\
$\beta_{p}$ & 0.001 \\
$\beta_{t}(\%)$ & 0.060 \\
\hline \hline
\end{tabular}

Table A.5: Data for coil groups OHL (T) and $\mathrm{OH} L(\mathrm{~B})$, EFL (T) and $\mathrm{EFL}(\mathrm{B}), \mathrm{EF} 2$ ('T) and $\mathrm{EF}_{2}(\mathrm{~B})$, and EF3 (T) and EF3 (B) carrying symetric currents.

\begin{tabular}{|c|c|c|c|c|c|}
\hline & Symmetric & Asymmetric & & Symmetric: & Asymmetris \\
\hline Coil & \multicolumn{2}{|c|}{ Current (kA) } & Coil & \multicolumn{2}{|c|}{ Current (kA.) } \\
\hline $\mathrm{OH} 1(\mathrm{~T})$ & 20.6655 & 20.665 & $\mathrm{OH} 1$ (B) & 20.6665 & 20.6665 \\
\hline $\mathrm{EF} 1(\mathrm{~T})$ & $84 .+24$ & 84.42 .4 & EF1 (B) & $8.4+2.4$ & $8.4 .+2.4$ \\
\hline $\mathrm{EF} 2(\mathrm{~T})$ & 35.775 & 35.775 & EF2 (B) & 35.775 & 35.775 \\
\hline \multirow[t]{2}{*}{$\mathrm{EF} 3(\mathrm{~T})$} & -17.739 & -17.739 & EF3 (B) & -17.739 & -17.7399 \\
\hline & \multicolumn{2}{|c|}{ Error $(\%)$} & & & \\
\hline Field Error & $9.318: 33$ & 9.318 .33 & & & \\
\hline
\end{tabular}


Table A.6: Data for coils OH1 (T), OH2 (B), EF1 (T), EF1 (B), EF2 (T), EF2 (B), EF3 (T), and EF3 (B) carrying unique currents.

\begin{tabular}{|c|c|c|c|c|c|}
\hline & Symmetric & Asymmetric & & Symmetric & Asymmetric \\
\hline Coil & \multicolumn{2}{|c|}{ Current (kA) } & Coil & \multicolumn{2}{|c|}{ Current (kA) } \\
\hline $\mathrm{OH} 1(\mathrm{~T})$ & 20.665 & 20.665 & $\mathrm{OH} 1(\mathrm{~B})$ & 20.665 & 20.665 \\
\hline $\mathrm{EF} 1(\mathrm{~T})$ & 84.424 & 84.424 & $\mathrm{EF} 1(\mathrm{~B})$ & 84.424 & 84.424 \\
\hline $\mathrm{EF} 2(\mathrm{~T})$ & 35.775 & 35.775 & $\mathrm{EF} 2$ (B) & 35.775 & 35.775 \\
\hline \multirow[t]{2}{*}{ EF3 (T) } & -17.739 & -17.739 & EF3 (B) & -17.739 & -17.739 \\
\hline & \multicolumn{2}{|c|}{ Error (\%) } & & & \\
\hline Field Error & 9.31833 & 9.31833 & & & \\
\hline
\end{tabular}

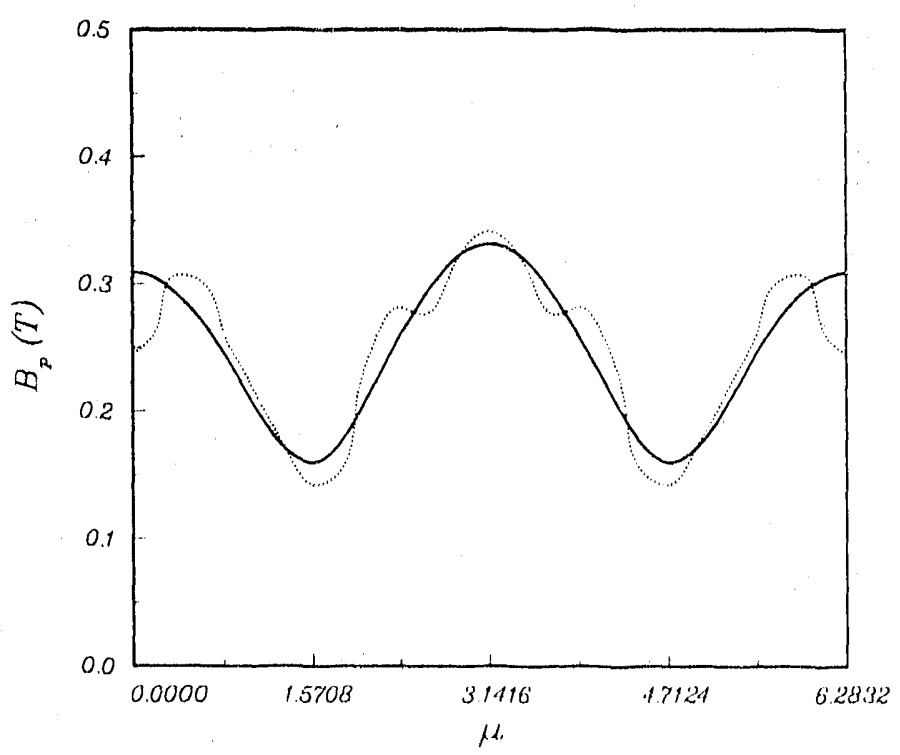

Figure A-4: Field comparison for the highly elongated tokamak case. The rquilibrium poloidal field of the plasma is the solid line. The dot ted line is the best obtainable match to the equilibrium using this algorithm. 


\section{Appendix B}

\section{Alcator C-Mod Coil Set}

This appendix lists the locations of each of the wires making up the 1.3 coils of the Alcator C-Mod Coil Set used in Chapters 3, 4, and 6 for testing the modifications to CPFC.

The wires for the EF1U and EF1L coils are listed in Tabic B.1. Each of these wires is $0.0227 \mathrm{~m}$ in thickness and $0.0237 \mathrm{~m}$ in height.

Table B.2 lists the locations or the wires for the EF2U and EF2L coils. Each of the wires has a radial thickness of $0.0233 \mathrm{~m}$ and an axial height of $0.0237 \mathrm{~m}$.

Each of the wires listed in Table B.3 for the EF3U and EF3L coils has a radial width of $0.0252 \mathrm{~m}$ and a height of $0.0240 \mathrm{~m}$.

Table B.4 lists the wire positions making up the EFCU and EFCL coils. These coils are used to control the vertical position of the plasma. Each of the wires making up these coils is $0.0191 \mathrm{~m}$ wide by $0.0247 \mathrm{~m}$ high.

The wires listed in Table B.5 comprise the EF4U and EF4L coils. Each of these wires is $0.0227 \mathrm{~m}$ wide and $0.0237 \mathrm{~m}$ high.

The wires making up the upper and lower segments of the $\mathrm{OH} 2$ coil are listed in Table B.6. These wires measure $0.017 .4 \mathrm{~m}$ in width by $0.02 .40 \mathrm{~m}$ in height.

Finally, the wires comprising the ohmic transformer (the OHI coil) are listed in Table B.7. Each of these wires measures $0.017 .4 \mathrm{~m}$ in the radial direction by $0.0240 \mathrm{~m}$ in the axial direction. 
Table B.1: EF1U and EF1L wire locations.

\begin{tabular}{|c|c|c|l|c|c|}
\hline \hline \multicolumn{3}{|c|}{ EF1U } & \multicolumn{3}{c|}{ EF1L } \\
\hline Wire & R (m) & Z (m) & Wire & R (m) & Z (m) \\
\hline EF1U_01 & 0.4457 & 0.5585 & EF1L_01 & 0.4457 & -0.5585 \\
EF1U_02 & 0.4684 & 0.5585 & EF1L_02 & 0.4684 & -0.5585 \\
EF1U_03 & 0.4912 & 0.5585 & EF1L_03 & 0.4912 & -0.5585 \\
EF1U_04 & 0.5139 & 0.5585 & EF1L_04 & 0.5139 & -0.5585 \\
EF1U_05 & 0.4457 & 0.5822 & EF1L_05 & 0.4457 & -0.5822 \\
EF1U_06 & 0.4684 & 0.5822 & EF1L_06 & 0.4684 & -0.5822 \\
EF1U_07 & 0.4912 & 0.5822 & EF1L_07 & 0.4912 & -0.5822 \\
EF1U_08 & 0.5139 & 0.5822 & EF1L_08 & 0.5139 & -0.5822 \\
EF1U_09 & 0.4457 & 0.6060 & EF1L_09 & 0.4457 & -0.6060 \\
EF1U_10 & 0.4684 & 0.6060 & EF1L_10 & 0.4684 & -0.6060 \\
EF1U_11 & 0.4912 & 0.6060 & EF1L_11 & 0.4912 & -0.6060 \\
EF1U_12 & 0.5139 & 0.6060 & EF1L_12 & 0.5139 & -0.6060 \\
EF1U_13 & 0.4457 & 0.6297 & EF1L_13 & 0.4457 & -0.6297 \\
EF1U_14 & 0.4684 & 0.6297 & EF1L_14 & 0.4684 & -0.6297 \\
EF1U_15 & 0.4912 & 0.6297 & EF1L_15 & 0.4912 & -0.6297 \\
EF1U_16 & 0.5139 & 0.6297 & EF1L_16 & 0.5139 & -0.6297 \\
\hline \hline
\end{tabular}


Table B.2: EF2U and EF2L wire locations.

\begin{tabular}{|c|c|c|c|c|c|}
\hline \multicolumn{3}{|c|}{ EF2U } & \multicolumn{3}{c|}{ EF2L } \\
\hline Wire & R $(\mathrm{m})$ & $\mathrm{Z}(\mathrm{m})$ & Wire & R $(\mathrm{m})$ & $\mathrm{Z}(\mathrm{m})$ \\
\hline EF2U_01 & 0.8423 & 0.5585 & EF2L_01 & 0.8423 & -0.5585 \\
EF2U_02 & 0.8653 & 0.5585 & EF2L_02 & 0.8653 & -0.5585 \\
EF2U_03 & 0.8882 & 0.5585 & EF2L_03 & 0.8882 & -0.5585 \\
EF2U_04 & 0.9112 & 0.5585 & EF2L_04 & 0.9112 & -0.5585 \\
EF2U_05 & 0.8423 & 0.5822 & EF2L_05 & 0.8423 & -0.5822 \\
EF2U_06 & 0.8653 & 0.5822 & EF2L_06 & 0.8653 & -0.5822 \\
EF2U_07 & 0.8882 & 0.5822 & EF2L_07 & 0.8882 & -0.5822 \\
EF2U_08 & 0.9112 & 0.5822 & EF2L_08 & 0.9112 & -0.5822 \\
EF2U_09 & 0.8423 & 0.6060 & EF2L_09 & 0.8423 & -0.6060 \\
EF2U_10 & 0.8653 & 0.6060 & EF2L_10 & 0.86553 & -0.60660 \\
EF2U_11 & 0.8882 & 0.6060 & EF2L_11 & 0.8882 & -0.6060 \\
EF2U_12 & 0.9112 & 0.6060 & EF2L_12 & 0.9112 & -0.6060 \\
EF2U_13 & 0.8 .23 & 0.6297 & EF2L_13 & 0.8423 & -0.6297 \\
EF2U_14 & 0.8653 & 0.6297 & EF2L_14 & 0.8653 & -0.6297 \\
EF2U_15 & 0.8882 & 0.6297 & EF2L_15 & 0.8882 & -0.6297 \\
EF2U_16 & 0.9112 & 0.6297 & EF2L_16 & 0.9112 & -0.6297 \\
\hline \hline
\end{tabular}


Table B.3: EF3U and EF3L wire locations.

\begin{tabular}{|c|c|c|c|c|c|}
\hline \multicolumn{3}{|c|}{ EF3U } & \multicolumn{3}{|c|}{ EF3L } \\
\hline Wire & $\mathrm{R}(\mathrm{m})$ & $\mathrm{Z}(\mathrm{m})$ & Wire & $\mathrm{R}(\mathrm{m})$ & $\mathrm{Z}(\mathrm{m})$ \\
\hline EF3U_01 & 0.9678 & 0.3899 & EF3L_01 & 0.9678 & -0.3899 \\
\hline EF3U_02 & 0.9930 & 0.3899 & EF3L_02 & 0.9930 & -0.3899 \\
\hline EF3U_03 & 1.0182 & 0.3899 & EF3L_03 & 1.0182 & -0.3899 \\
\hline EF3U_04 & 1.0434 & 0.3899 & EF3L_()4 & 1.0434 & -0.3899 \\
\hline EF3U_05 & 0.9678 & 0.4139 & EF3L_05 & 0.9678 & -0.4139 \\
\hline EF3U_06 & 0.9930 & 0.4139 & EF3L_06 & 0.9930 & -0.4139 \\
\hline EF3U_07 & 1.0182 & 0.4139 & EF3L_07 & 1.01 .82 & -0.4139 \\
\hline EF3U_08 & 1.0434 & 0.4139 & EF3L_08 & 1.0434 & -0.4139 \\
\hline EF3U_09 & 0.9678 & 0.4379 & EF3L_09 & 0.9678 & -0.4379 \\
\hline EF3U_10 & 0.9930 & 0.4379 & EF3L_10 & 0.9930 & -0.4379 \\
\hline EF3U...1 1 & 1.0182 & 0.4379 & EF3L_11 & 1.0182 & -0.4379 \\
\hline EF3U_12 & 1.0434 & 0.4379 & EF3L_12 & 1.0434 & -0.4379 \\
\hline EF3U_13 & 0.9678 & 0.4619 & EF3L_13 & 0.9678 & -0.4619 \\
\hline EF3U_14 & 0.9930 & 0.4619 & EF3I._14 & 0.9930 & -0.4619 \\
\hline EF3U_15 & 1.0182 & 0.4619 & EF3L_15 & 1.0182 & -0.461 .9 \\
\hline EF3U..16 & 1.0434 & 0.4619 & EF3L_16 & 1.0434 & -0.4619 \\
\hline EF3U_17 & 0.9678 & 0.48 .59 & EF3L..17 & 0.9678 & -0.4859 \\
\hline EF3U_18 & 0.9930 & 0.4859 & EF3L_18 & 0.9930 & -0.4859 \\
\hline EF3U_19 & 1.0182 & 0.4859 & EF3L_19 & 1.0182 & -0.48 .59 \\
\hline EF3U_20 & 1.0434 & 0.4859 & EF3L_20 & 1.043 .4 & -0.4859 \\
\hline EF3U_21 & 0.9678 & 0.50999 & $\mathrm{EF} 3 \mathrm{~L} 21$ & 0.9678 & $-0.5(9) 99$ \\
\hline EF3U 22 & 0.99330 & 0.50999 & EF3L 22 & $0.99: 30$ & $-(0.50999$ \\
\hline $\mathrm{EF} 3 \mathrm{U} 23$ & 1.0182 & 0.50999 & EF3L 23 & $1.01 \times 2$ & $-0.50(999$ \\
\hline $\mathrm{EF} 3 \mathrm{U} 24$ & 1.043 .1 & 0.5099 & EF3L_24 & $1.04+3.4$ & -0.5099 \\
\hline
\end{tabular}


Table B.4: EFCU and EFCL wire locations.

\begin{tabular}{|c|c|c|c|c|c|}
\hline \hline \multicolumn{3}{|c|}{ EF4U } & \multicolumn{3}{c|}{ EF4L } \\
\hline Wire & $\mathrm{R}(\mathrm{m})$ & $\mathrm{Z}(\mathrm{m})$ & Wire & $\mathrm{R}(\mathrm{m})$ & $\mathrm{Z}(\mathrm{m})$ \\
\hline EFCU_01 & 0.8403 & 0.4178 & EFCL_01 & 0.8403 & -0.4178 \\
EFCU_02 & 0.8594 & 0.4178 & EFCL_02 & 0.8594 & -0.41 .78 \\
EFCU_03 & 0.8403 & 0.4425 & EFCL_03 & 0.83403 & -0.4425 \\
EFCU_04 & 0.8594 & 0.4425 & EFCL_04 & 0.8594 & -0.4425 \\
\hline \hline
\end{tabular}


Table B.5: EF4U and EF4L wire locations.

\begin{tabular}{|c|c|c|c|c|c|}
\hline \hline \multicolumn{3}{|c|}{ EF4U } & \multicolumn{3}{|c|}{ EF4L } \\
\hline Wire & $\mathrm{R}(\mathrm{m})$ & $\mathrm{Z}(\mathrm{m})$ & Wire & $\mathrm{R}(\mathrm{m})$ & $\mathrm{Z}(\mathrm{m})$ \\
\hline EF4U_01 & 1.4719 & 0.4107 & EF4L_01 & 1.4719 & -0.4107 \\
EF4U_02 & 1.4946 & 0.4107 & EF4L_02 & 1.4946 & -0.4107 \\
EF4U_03 & 1.5173 & 0.4107 & EF4L_03 & 1.5173 & -0.4107 \\
EF4U_04 & 1.5400 & 0.4107 & EF4L_04 & 1.5400 & -0.4107 \\
EF4U_05 & 1.4719 & 0.4344 & EF4L_05 & 1.1719 & -0.4344 \\
EF4U_06 & 1.4946 & 0.434 .4 & EF4L_06 & 1.4946 & -0.4344 \\
EF4U_07 & 1.5173 & 0.4344 & EF4L_07 & 1.5173 & -0.4344 \\
EF4U_08 & 1.5400 & 0.4344 & EF4L_08 & 1.54400 & -0.4344 \\
EF4U_09 & 1.4719 & 0.4582 & EF4L_09 & 1.4719 & -0.4582 \\
EF4U_10 & 1.4946 & 0.4582 & EF4L_10 & 1.4946 & -0.4582 \\
EF4U_11 & 1.5173 & 0.4582 & EF4L_11 & 1.5173 & -0.4582 \\
EF4U_12 & 1.5400 & 0.4582 & EF4L_12 & 1.5400 & -0.4582 \\
EF4U_13 & 1.4719 & 0.1819 & EF4L_13 & 1.4719 & -0.4819 \\
EF4U_14 & 1.4946 & 0.4819 & EF4L_14 & 1.4946 & -0.4819 \\
EF4U_15 & 1.5173 & 0.4819 & EF4L_15 & 1.5173 & -0.4819 \\
EF4U_16 & 1.54100 & 0.4819 & EF4L_16 & 1.54100 & -0.1819 \\
\hline \hline
\end{tabular}


Table B.6: OH2U and OH2L wire locations.

\begin{tabular}{|c|c|c|c|c|c|}
\hline \hline \multicolumn{3}{|c|}{ OH2U } & \multicolumn{3}{|c|}{ OH2L } \\
\hline Wire & $\mathrm{R}(\mathrm{m})$ & $\mathrm{Z}(\mathrm{m})$ & Wire & $\mathrm{R}(\mathrm{m})$ & $\mathrm{Z}(\mathrm{m})$ \\
\hline OH2U_01 & 0.3584 & 0.2637 & OH2L_01 & 0.3584 & -0.2637 \\
OH2U_02 & 0.3584 & 0.2877 & OH2L_02 & 0.3584 & -0.2877 \\
OH2U_03 & 0.3584 & 0.3117 & OH2L_03 & 0.3584 & -0.31 .17 \\
OH2U_04 & 0.3584 & 0.3356 & OH2L_04 & 0.3584 & -0.3356 \\
OH2U_05 & 0.3584 & 0.3596 & OH2L_05 & 0.3584 & -0.3596 \\
OH2U_06 & 0.3584 & 0.3836 & OH2L_06 & 0.3584 & -0.3836 \\
OH2U_07 & 0.3584 & 0.4075 & OH2L_07 & 0.3584 & -0.4075 \\
OH2U_08 & 0.3584 & 0.4315 & OH2L_08 & 0.3584 & -0.4315 \\
OH2U_09 & 0.3584 & 0.4555 & OH2L_09 & 0.3584 & -0.4555 \\
OH2U_10 & 0.3584 & 0.4795 & OH2L_10 & 0.3584 & -0.4795 \\
OH2U_11 & 0.3584 & 0.5034 & OH2L_11 & 0.3584 & -0.5034 \\
OH2U_12 & 0.3584 & 0.5774 & OH2L_12 & 0.3584 & -0.5274 \\
OH2U_13 & 0.3584 & 0.5514 & OH2L_13 & 0.3584 & -0.5514 \\
OH2U_14 & 0.3758 & 0.2397 & OH2L_14 & 0.3758 & -0.2397 \\
OH2U_15 & 0.3758 & 0.2637 & OH2L_15 & 0.3758 & -0.2637 \\
OH2U_16 & 0.3758 & 0.2877 & OH2L_16 & 0.3758 & -0.2877 \\
OH2U_17 & 0.3758 & 0.3117 & OH2L_17 & 0.3758 & -0.3117 \\
OH2U_18 & 0.3758 & 0.3356 & OH2L_18 & 0.3758 & -0.3356 \\
OH2U_19 & 0.3758 & 0.3596 & OH2L_19 & 0.3758 & -0.3596 \\
OH2U20 & 0.3758 & 0.3836 & OH2L_20 & 0.3758 & -0.38336 \\
OH2U_21 & 0.3758 & 0.4075 & OH2L_21 & 0.3758 & -0.4075 \\
OH2U22 & 0.3758 & 0.4315 & OH2L_22 & 0.3758 & -0.4315 \\
OH2U_23 & 0.3758 & 0.4555 & OH2L_23 & 0.3758 & $-0.4555)$ \\
OH2U_24 & 0.3758 & 0.4795 & OH2L_24 & 0.3758 & -0.4795 \\
OH2U_25 & 0.3758 & 0.5034 & OH2L_25 & 0.3758 & -0.50334 \\
OH2U_26 & 0.3758 & 0.5274 & OH2L_26 & 0.3758 & -0.527. \\
OH2U_27 & 0.3758 & 0.5514 & OH2L_27 & 0.3758 & -0.551. \\
\hline \hline
\end{tabular}


Table B.7: Wire locations of the OHI ohmic transformer.

\begin{tabular}{|c|c|c|c|c|c|}
\hline \multicolumn{6}{|c|}{$\mathrm{OH} 1$} \\
\hline Wire & $\mathrm{R}(\mathrm{m})$ & $\mathrm{Z}(\mathrm{m})$ & Wire & $\mathrm{R}(\mathrm{m})$ & $\mathrm{Z}(\mathrm{m})$ \\
\hline OH1_OO1 & 0.3236 & -0.5514 & OH1_OO2 & 0.3236 & -0.5274 \\
\hline $\mathrm{OH} 1.003$ & 0.3236 & -0.5034 & OH1_004 & 0.3236 & -0.4795 \\
\hline OH1_005 & 0.3236 & -0.4555 & OH1.006 & 0.3236 & -0.4315 \\
\hline OH1_007 & 0.3236 & -0.4075 & OH1_008 & 0.3236 & -0.3836 \\
\hline OH1_009 & 0.3236 & -0.3596 & OH1_010 & 0.3236 & -0.33356 \\
\hline OH1_011 & 0.3236 & -0.3117 & $\mathrm{OH} 1 \_012$ & 0.3236 & -0.2877 \\
\hline OH1_013 & 0.3236 & -0.2637 & $\mathrm{OH} 1 . .014$ & 0.3236 & -0.2397 \\
\hline OH1_015 & 0.3236 & -0.2158 & OH1_016 & 0.3236 & -0.1918 \\
\hline OH1_017 & 0.3236 & -0.1678 & OH1_018 & 0.3236 & -0.1438 \\
\hline OH1_019 & 0.3236 & -0.1199 & $\mathrm{OH} 1 \_020$ & 0.3236 & -0.09599 \\
\hline OH1_021 & 0.3236 & -0.0719 & $\mathrm{OH} 1 \_022$ & 0.3236 & -0.0479 \\
\hline $\mathrm{OH} 1 \_023$ & 0.3236 & -0.0240 & $\mathrm{OH} 1 \_024$ & 0.3236 & +0.0000 \\
\hline OH1_025 & 0.3236 & +0.0240 & OH1_026 & 0.3236 & +0.0479 \\
\hline $\mathrm{OH} 1 \_027$ & 0.3236 & +0.0719 & OH1_028 & 0.3236 & $+0 .(0959)$ \\
\hline OH1_029 & 0.3236 & +0.1199 & $\mathrm{OH} 1 \_030$ & 0.3236 & +0.1438 \\
\hline OH1_031 & 0.3236 & +0.1678 & $\mathrm{OH} 1 \_032$ & 0.32336 & +0.1918 \\
\hline OH1_(O33 & 0.3236 & +0.2158 & OH1_034 & 0.32336 & +0.2397 \\
\hline OH1_O35 & 0.3236 & $+0.263 \mathrm{~T}$ & OH1_036 & 0.3236 & $+0.287 T$ \\
\hline OH1..037 & 0.3236 & +0.3117 & OH1_038 & $0.32: 36$ & +0.33356 \\
\hline OH1_039 & 0.3236 & +0.3596 & OH1_()40 & $0.32: 36$ & +0.3836 \\
\hline $\mathrm{OH} 1 \_041$ & 0.3236 & +0.4075 & $\mathrm{OH} 1 \_042$ & 0.3236 & +0.431 .5 \\
\hline OH1_043 & 0.3236 & +0.4555 & OH1_044 & $0.32: 36$ & +0.179 .5 \\
\hline OH1_045 & 0.3236 & +0.5034 & $\mathrm{OH} 1 \_046$ & 0.3236 & +0.5274 \\
\hline OH1_047 & $0.3236^{\circ}$ & +0.5514 & OH1_O48 & 0.3410 & -0.551 .1 \\
\hline OH1_049 & 0.3410 & -0.527 .1 & OHL_O50 & $0.3+110$ & -0.50334 \\
\hline
\end{tabular}


Table B.7: Continued

\begin{tabular}{|c|c|c|c|c|c|}
\hline \multicolumn{6}{|c|}{$\mathrm{OH} 1$} \\
\hline Wire & $\mathrm{R}(\mathrm{m})$ & $\mathrm{Z}(\mathrm{m})$ & Wìre & $\mathrm{R}(\mathrm{m})$ & $\mathrm{Z}(\mathrm{m})$ \\
\hline $\mathrm{OH} 1 \_051$ & 0.3410 & -0.4735 & $\mathrm{OH} 1 \_052$ & 0.3410 & -0.4555 \\
\hline OH1_053 & 0.3410 & -0.4315 & $\mathrm{OH} 1 \_054$ & 0.3410 & -0.4075 \\
\hline OH1_055 & 0.3410 & -0.3836 & OH1_056 & 0.3410 & -0.3596 \\
\hline OH1_057 & 0.3410 & -0.3356 & OH1_.058 & 0.3410 & -0.3117 \\
\hline OH1_059 & 0.3410 & -0.2877 & OH1_060 & 0.3410 & -0.2637 \\
\hline $\mathrm{OH} 1_{-} 061$ & 0.3410 & -0.2397 & OH1_062 & 0.3410 & -0.2158 \\
\hline OH1_063 & 0.3410 & -0.1918 & $\mathrm{OH} 1 \_064$ & 0.3410 & -0.1678 \\
\hline OH1_065 & 0.3410 & -0.1438 & OH1_066 & 0.3410 & -0.1199 \\
\hline OH1_067 & 0.3410 & -0.0959 & OH1_068 & 0.3410 & -0.0719 \\
\hline OH1_069 & 0.3410 & -0.0480 & OH1_070 & 0.3410 & -0.0240 \\
\hline $\mathrm{OH} 1.071$ & 0.3410 & +0.0000 & OH1_072 & 0.3410 & +0.0240 \\
\hline OH1_073 & 0.3410 & +0.0479 & OH1_074' & 0.3410 & +0.0719 \\
\hline OH1_075 & 0.3410 & +0.0959 & OH1_076 & 0.3410 & +0.1199 \\
\hline OH1_077 & 0.3410 & +0.1438 & OH1_078 & 0.3410 & +0.1678 \\
\hline OH1_079 & 0.3410 & +0.1918 & OH1_080 & $0.3+10$ & +0.2277 \\
\hline OH1_081 & 0.3410 & +0.2517 & OH1_O82 & 0.3 .410 & +0.2757 \\
\hline OH1_083 & 0.3410 & +0.2997 & OH1_084 & $0.3+110$ & +0.3236 \\
\hline OH1_085 & 0.3410 & +0.3476 & OH1_086 & $0.3 \times 110$ & +0.3716 \\
\hline OH1_087 & 0.34110 & +0.3955 & $\mathrm{OH} 1 \_088$ & $0.3+1.0$ & +0.4195 \\
\hline OH1_089 & 0.3410 & +0.44355 & $\mathrm{OH}{ }_{1}(090)$ & $0.3+10$ & +0.4675 \\
\hline $\mathrm{OH} 1 \_091$ & 0.3410 & +0.491 .1 & OH1_(O92 & 0.3 .110 & +0.515 .1 \\
\hline OH1_(093 & 0.3410 & +0.53934 & OH1_(094 & 0.34110 & +0.5754 \\
\hline OH1_095 & 0.358 .4 & -0.1918 & $\mathrm{OH} 1 \_096$ & $0.355 \times 4$ & -0.1678 \\
\hline OH1_(097 & 0.358 .84 & $-0.1+138$ & OHI_(988 & 0.358 .1 & -0.1199 \\
\hline $\mathrm{OH} 1 \_099$ & 0.358 .4 & -0.0959 & $\mathrm{OH} 1_{-} 1(0)$ & 0.358 .1 & -0.0719 \\
\hline
\end{tabular}


Table B.7: Continued

\begin{tabular}{|c|c|c|c|c|c|}
\hline \multicolumn{6}{|c|}{$\mathrm{OH} 1$} \\
\hline Wire & $\mathrm{R}(\mathrm{m})$ & $\mathrm{Z}(\mathrm{m})$ & Wire & $\mathrm{R}(\mathrm{m})$ & $\mathrm{Z}(\mathrm{m})$ \\
\hline OH1..101 & 0.3584 & -0.0479 & $\mathrm{OH} 1 \_102$ & 0.3584 & $\cdots(0.0)^{\circ} 40$ \\
\hline OH1_103 & 0.3584 & +0.0000 & $\mathrm{OH} 1,-104$ & 0.3584 & +0.0240 \\
\hline $\mathrm{OH} 1 \_105$ & 0.3584 & +0.0479 & $\mathrm{OH} 1 \_106$ & 0.3584 & +0.0719 \\
\hline OH1_107 & 0.3584 & +0.0959 & $\mathrm{OH} 1 \_108$ & 0.3584 & +0.1199 \\
\hline OH1_109 & 0.3584 & +0.1438 & $\mathrm{OH}_{-} \_110$ & 0.3584 & +0.1678 \\
\hline OH1_111 & 0.3584 & +0.1918 & $\mathrm{OH} 1.112$ & 0.3758 & -0.2038 \\
\hline $\mathrm{OH} 1 \_113$ & 0.3758 & -0.1798 & $\mathrm{OH} 1.1 \| 4$ & 0.3758 & $-(0.1558$ \\
\hline OH1_115 & 0.3758 & -0.1319 & $\mathrm{OH} 1-\mathrm{il}$ & 0.3758 & -0.1079 \\
\hline OH1_117 & 0.3758 & -0.0839 & $\mathrm{OH} 1.118$ & 0.3758 & -0.05599 \\
\hline $\mathrm{OH} 1 \_119$ & 0.3758 & -0.0360 & $\mathrm{OH} 1 \_120$ & 0.3758 & -0.0120 \\
\hline $\mathrm{OH} 1 \_121$ & 0.3758 & +0.0120 & $\mathrm{OH} 1 \ldots 2$ & 0.3758 & +0.0360 \\
\hline $\mathrm{OH} 1 \_123$ & 0.3758 & +0.0599 & $\mathrm{OH} 1 \ldots 124$ & 0.3758 & +0.08 .39 \\
\hline $\mathrm{OH} 1 \_125$ & 0.3758 & +0.1079 & OH1_126 & 0.3758 & +0.1319 \\
\hline OH1_127 & 0.3758 & +0.1558 & $\mathrm{OH} 1 \_128$ & 0.3758 & +0.17 .98 \\
\hline $\mathrm{OH} 1 \ldots 129$ & 0.3758 & +0.2038 & & & \\
\hline
\end{tabular}




\section{Appendix C}

\section{Symmetry Relations}

In this appendix, various symmetry relations for the function $\left(i\left(r, z ; r^{\prime}, z^{\prime}\right)\right.$ are developed. These relations are then used to demonstrate the symmetry of the energy $I_{j},-$ which, in turn, is used to demonstrate the symmetry of the mutual inductance $M_{i j}$.

\section{C.1 Symmetry of $G\left(r, z ; r^{\prime}, z^{\prime}\right)$}

In this section, the symmetry of the function

$$
\left(i\left(r, z ; r^{\prime}, z^{\prime}\right)\right.
$$

with respect to the exchange of variables

$$
\begin{aligned}
& r \longrightarrow r^{\prime} \\
& z-z^{\prime}
\end{aligned}
$$

will be demonstrated. The function $\left(r\left(r, z ; r^{\prime}, z^{\prime}\right)\right.$ is defined as

$$
\left(i\left(r, z r^{\prime}, z^{\prime}\right)=\frac{2.1}{r r^{\prime}}\left[\left(1-\frac{k^{2}}{2}\right) h\left(k_{i}\right)-E^{\prime}\left(k_{i}\right)\right]\right.
$$

where

$$
\begin{aligned}
& A^{2}=\left(r+r^{\prime}\right)^{2}+\left(z-z^{\prime}\right)^{2} \\
& k^{2}=\frac{4 r^{\prime}}{d^{2}}
\end{aligned}
$$

so that by demonstrating the symmetry of $A$ and $k$, the symmetry of $(i$ will follow. 


\section{C.1.1 Exchange of $r$ and $r^{\prime}$}

Under the exchange of $r$ and $r^{\prime}$, we obtain:

$$
\begin{aligned}
A^{\prime 2} & =\left(r^{\prime}+r^{\prime 2}+\left(z-z^{\prime}\right)^{2}\right. \\
& =\left(r+r^{\prime}\right)^{2}+\left(z-z^{\prime}\right)^{2} \\
& =A^{2}
\end{aligned}
$$

and

$$
\begin{aligned}
k^{\prime 2} & =\frac{4 r^{\prime} r}{A^{\prime 2}} \\
& =\frac{4 r^{\prime}}{A^{2}} \\
& =k^{2}
\end{aligned}
$$

so that

$$
\begin{aligned}
G^{\prime}\left(r^{\prime}, z ; r, z^{\prime}\right) & =\frac{2 A^{\prime}}{r^{\prime} r}\left[\left(1-\frac{k^{\prime 2}}{2}\right) K^{\prime}\left(k^{\prime}\right)-E\left(k^{\prime}\right)\right] \\
& =\frac{2 A}{r^{\prime}}\left[\left(1-\frac{k^{2}}{2}\right) H^{\prime}(k)-E\left(k_{i}\right)\right] \\
& =C^{\prime}\left(r, z ; r^{\prime}, z^{\prime}\right)
\end{aligned}
$$

\section{C.1.2 Exchange of $z$ and $z^{\prime}$}

The exchange of $z$ with $z^{\prime}$ proceeds in a similar manner:

$$
\begin{aligned}
A^{\prime 2} & =\left(r+r^{\prime}\right)^{2}+\left(z^{\prime}-z\right)^{2} \\
& =\left(r+r^{\prime}\right)^{2}+(-1)^{2}\left(z-z^{\prime}\right)^{2} \\
& =\left(r+r^{\prime}\right)^{2}+\left(z-z^{\prime}\right)^{2} \\
& =A^{2}
\end{aligned}
$$

and

$$
k^{\prime 2}=\frac{4 r r^{\prime}}{d^{\prime 2}}=\frac{4 r r^{\prime}}{d^{2}}=k^{2}
$$

resulting in

$$
\left(i\left(r, z^{\prime}: r^{\prime}, z\right)=\left(i\left(r, z ; r^{\prime}, z^{\prime}\right)\right.\right.
$$




\section{C.1.3 Results}

Quoting the previous results:

$$
\begin{aligned}
\left(r\left(r, z ; r^{\prime}, z^{\prime}\right)\right. & =\left(r\left(r^{\prime}, z ; r, z^{\prime}\right)\right. \\
& =\left(r^{\prime}\left(r, z^{\prime} ; r^{\prime}, z\right)\right.
\end{aligned}
$$

and, by extension,

$$
C\left(r, z ; r^{\prime}, z^{\prime}\right)=\left(r^{\prime}\left(r^{\prime}, z^{\prime} ; r, z\right)\right.
$$

\section{C.2 Symmetry of $U_{j-i}$}

In this section, the symmetry relation

$$
U_{j \rightarrow i}=I_{i \rightarrow j}
$$

will be demonstrated. The full expression for $U_{j-i}$ is given by

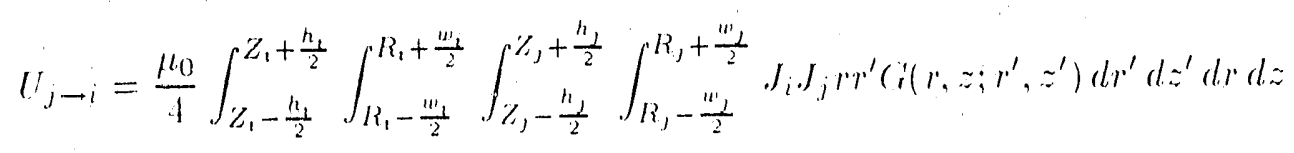

Exchanging the subscripts $i$ and $j$ gives

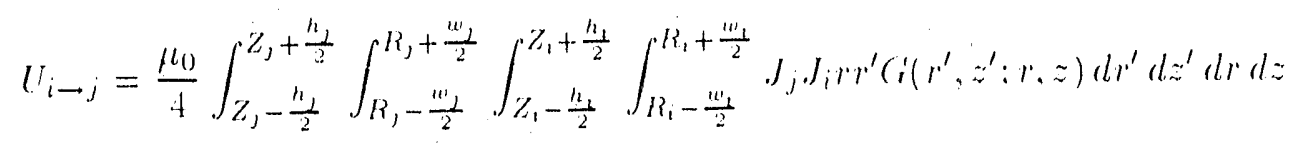

Now, rearranging the order of integration results in

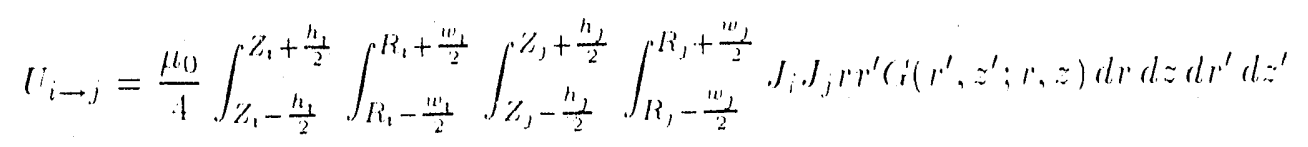

Now, making the exchange of variables

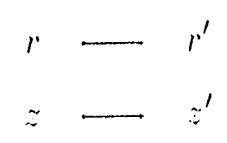

and making use of the symmetry relations for ( $i$ developed in the previous section gives

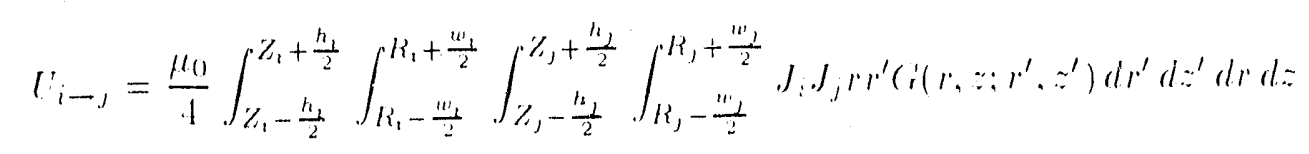

where the right-hand side is identical to the right-hand side of equation C.1 so that

$$
I_{1 \rightarrow i}=l_{i \rightarrow 1}
$$




\section{C.3 Symmetry of $M_{i j}$}

In this section the symmetry relation

$$
M_{i j}=M_{j i}
$$

is demonstrated.

The mutual inductance $M_{i j}$ is defined as

$$
M_{i j}=\frac{2 U_{i j \rightarrow i}}{I_{i} I_{j}}
$$

Exchanging the subscripts $i$ and $j$ results in

$$
\begin{aligned}
M_{j i} & =\frac{2 U_{i \rightarrow j}}{I_{j} I_{i}} \\
& =\frac{2 U_{j \rightarrow i}}{I_{i} I_{j}} \\
& =M_{i j}
\end{aligned}
$$

where use has been made of the symmetry of the encrgy (demonstrated in the previous section). 


\section{Appendix D}

\section{Derivation of the Intercoil Force Expressions}

The expression for the force on coil $i$ due to all other coils can be easily compute from

$$
\mathbf{F}=-\nabla_{i} U_{t}
$$

where $U_{t}$ is the total magnetic energy of the system. The total energy, in turn, can be written as

$$
U_{t}=\sum_{i=1}^{N} \sum_{j=1}^{N} U_{j \rightarrow i}
$$

where $N$ is the total number of coils in the system and $U_{j \rightarrow i}$ is the magnetic energy associated with the two components $i$ and $j$. For the simple case of $N=2$, this gives

$$
U_{t}=U_{1 \rightarrow 1}+U_{2 \rightarrow 1}+U_{1 \rightarrow 2}+U_{2-2}
$$

Since

$$
U_{i \rightarrow 1}=U_{1 \rightarrow 1}
$$

the iotal energy of the system is given by

$$
U_{1}=U_{1 \rightarrow 1}+2 U_{2 \rightarrow 1}+U_{2-2}
$$

Taking the gradient of the energy with respect to coil 1 gives

$$
\nabla_{1} l_{t}=\nabla_{1} l_{1-1}+2 \nabla_{1} l_{2-i}
$$


The $\nabla_{1} U_{1 \rightarrow 1}$ gives the self-force of the coil, and the $\nabla_{1} U_{2 \rightarrow 1}$ term yields the interaction force on coil 1 due to coil 2 .

In this appendix, the explicit relations used to compute the forces on a coil are derived from the magnetic energy. This will be carried out first for the filamentary current case, and then for the more general case of distributed currents.

\section{D.1 Filamentary Currents}

\section{D.1.1 Force Between Two Coils}

The interaction energy of a filamentary current $I_{i}$ in the magnetic ficld produced by another filamentary current $I_{j}$ is given by

$$
U_{j \rightarrow i}=\frac{\mu_{0} I_{i} I_{j}}{4} R_{i} R_{j} C\left(R_{i}, Z_{i} ; R_{j}, Z_{j}\right)
$$

where

$$
\begin{aligned}
G\left(R_{i}, Z_{i} ; R_{j}, Z_{j}\right) & =\frac{2 A}{R_{i} R_{j}}\left[\left(1-\frac{k^{2}}{2}\right) K(k)-E(k)\right] \\
A^{2} & =\left(R_{i}+R_{j}\right)^{2}+\left(Z_{i}-Z_{i j}\right)^{2} \\
k^{2} & =\frac{4 R_{i} R_{j}}{A^{2}}
\end{aligned}
$$

The $\nabla_{i}$ operator is given by

$$
\nabla_{i}=\mathbf{e}_{\mathbf{R}} \frac{\partial}{\partial R_{i}}+\mathbf{e}_{\mathbf{Z}} \frac{\partial}{\partial Z_{i}}
$$

where the $\mathbf{e}_{\phi}$ component has been neglected due to toroidal symmetry. This gives

$$
\mathbf{F}=\mathbf{e}_{\mathbf{R}} \frac{\partial U_{j \rightarrow i}}{\partial R_{i}}+\mathbf{e}_{\mathbf{Z}} \frac{\partial U_{j \rightarrow i}}{\partial Z_{i}}
$$

Concentrating on the $\mathbf{e}_{\mathbf{R}}$ component first yields

$$
\begin{aligned}
\frac{\partial U j \rightarrow i}{\partial R_{i}}= & \frac{\mu_{0} I_{i} I_{j}}{2} \frac{\partial}{\partial R_{i}}\left\{A\left[\left(1-\frac{k^{2}}{2}\right) h\left(k_{i}\right)-E\left(k_{i}\right)\right]\right\} \\
= & \frac{\mu_{0} I_{i} I_{j}}{2}\left\{\frac{\partial A}{\partial R_{i}}\left[\left(1-\frac{k^{2}}{2}\right) h\left(k_{i}\right)-E_{(}\left(k_{i}\right)\right]\right. \\
& \left.+A \frac{\partial}{\partial k_{i}}\left[\left(1-\frac{k^{2}}{2}\right) h\left(k_{i}\right)-E^{\prime}\left(k_{i}\right)\right] \frac{\partial k_{i}}{\partial R_{i}}\right\}
\end{aligned}
$$


From the definitions of $A$ and $k$ given above,

$$
\begin{aligned}
& \frac{\partial A}{\partial R_{i}}=\frac{R_{i}+R_{j}}{A} \\
& \frac{\partial k}{\partial R_{i}}=\frac{2 A^{2} R_{j}-4 R_{i} R_{j}\left(R_{i}+R_{j}\right)}{k A^{4}}
\end{aligned}
$$

Next,

$$
\frac{\partial}{\partial k}\left[\left(1-\frac{k^{2}}{2}\right) K(k)-E(k)\right]=-k K(k)+\left(1-\frac{k^{2}}{2}\right) \frac{\partial K(k)}{\partial k}-\frac{\partial E(k)}{\partial k}
$$

The derivatives of the elliptic integrals $K^{\prime \prime}(k)$ and $E(k)$ are given by the identities

$$
\begin{aligned}
& \frac{\partial K^{\circ}(k)}{\partial k}=\frac{E(k)}{k\left(1-k^{2}\right)}-\frac{K(k)}{k} \\
& \frac{\partial E(k)}{\partial k}=\frac{E(k)-K(k)}{k}
\end{aligned}
$$

Substituting the elliptic integral derivatives and simplifying results in

$$
\frac{\partial}{\partial k}\left[\left(1-\frac{k^{2}}{2}\right) K^{\prime \prime}(k)-E(k)\right]=\frac{k}{2}\left[\frac{E(k)}{1-k^{2}}-K(k)\right]
$$

Incorporating this result and the partial derivatives of $A$ and $k$ into the derivative of the energy gives

$$
\begin{aligned}
\frac{\partial U_{j \rightarrow i}}{\partial R_{i}}= & \frac{\mu_{0} I_{i} I_{j}}{2} \frac{R_{i}+R_{j}}{A}\left\{\left[\left(1-\frac{k^{2}}{2}\right) h\left(k_{i}\right)-E\left(k_{i}\right)\right]\right. \\
& \left.+\left(\frac{R_{j}}{R_{i}+R_{j}}-\frac{k_{i}^{2}}{2}\right)\left[\frac{E\left(k_{i}\right)}{1-k^{2}}-K\left(k_{i}\right)\right]\right\}
\end{aligned}
$$

Using the definition of $k^{2}$ and simplifying results in

$$
\frac{\partial U_{j-i}}{\partial R_{i}}=\frac{\mu_{0} I_{i} I_{j}}{2} \frac{R_{i}+R_{j}}{A}\left\{\Lambda(k)\left[\frac{R_{i}}{R_{i}+R_{j}}\right]+\frac{E\left(k_{i}\right)}{1-k^{2}}\left[\frac{k^{2}}{2}-\frac{R_{i}}{R_{i}+R_{j}}\right]\right\}
$$

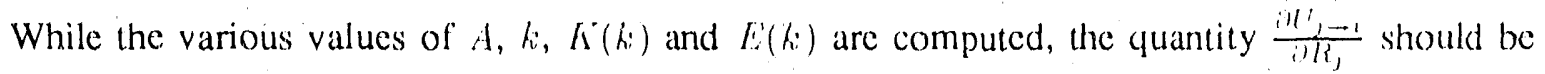
computed. The resulting expression is given by

$$
\frac{\partial I_{j \rightarrow i}}{\partial R_{j}}=\frac{\mu_{0} I_{i} I_{j}}{2} \frac{R_{i}+R_{j}}{A}\left\{h\left(k_{i}\right)\left[\frac{R_{j}}{R_{i}+R_{j}}\right]+\frac{L_{i}\left(k_{i}\right)}{1-k_{i}^{2}}\left[\frac{k_{i}^{2}}{2}-\frac{R_{1}}{R_{i}+R_{j}}\right]\right\}
$$

The derivation of the $\mathbf{e}_{\mathrm{Z}}$ component of the force is similar. Taking the $/{ }_{i}$ derivatives of $A$ and $k$,

$$
\begin{aligned}
& \frac{\partial A}{\partial Z_{i}}=\frac{Z_{1}-Z_{j}}{A} \\
& \frac{\partial k_{i}}{\partial Z_{i}}=-\frac{\mid R_{i} R_{j}\left(Z_{i}-Z_{1}\right)}{h_{i} A^{4}}
\end{aligned}
$$


The $Z_{i}$ derivative of the energy is then given by

$$
\frac{\partial U_{j \rightarrow i}}{\partial Z_{i}}=\frac{\mu_{0} I_{i} I_{j}}{2} \frac{Z_{i}-Z_{j}}{A}\left\{\left[\left(1-\frac{k^{2}}{2}\right) K(k)-E\left(k_{i}\right)\right]-\frac{2 R_{i} R_{j}}{A^{2}}\left[\frac{E\left(k_{i}\right)}{1-k^{2}}-K(k)\right]\right\}
$$

Simplifying this expression results in

$$
\frac{\partial U_{j \rightarrow i}}{\partial Z_{i}}=\frac{\mu_{0} I_{i} I_{j}}{2} \frac{Z_{i}-Z_{j}}{A}\left[K(k)+\frac{E(k)}{1-k^{2}}\left(\frac{k^{2}}{2}-1\right)\right]
$$

For the case of the $\mathrm{e}_{\mathrm{Z}}$ component, it can be shown that

$$
\frac{\partial U_{j \rightarrow i}}{\partial Z_{j}}=-\frac{\partial U_{j \rightarrow i}}{\partial Z_{i}}
$$

Thus, the total force on coil $i$ due to coil $j$ is given by

$$
\begin{aligned}
\mathbf{F}_{\mathrm{j} \rightarrow \mathrm{i}} & =\mu_{0} I_{i} I_{j} \frac{R_{i}+R_{j}}{A}\left\{K(k)\left[\frac{R_{i}}{R_{i}+R_{j}}\right]+\frac{E(k)}{1-k^{2}}\left[\frac{k^{2}}{2}-\frac{R_{i}}{R_{i}+R_{i}}\right]\right\} \mathbf{e}_{\mathbf{R}} \\
& +\mu_{0} I_{i} I_{j} \frac{Z_{i}-Z_{j}}{A}\left\{K(k)+\frac{E(k)}{1-k^{2}}\left(\frac{k^{2}}{2}-1\right)\right\} \mathbf{e}_{\mathbf{Z}}
\end{aligned}
$$

and the force on coil $j$ due to coil $i$ is given by

$$
\begin{aligned}
\mathrm{F}_{\mathrm{l} \rightarrow \mathrm{j}} & =\mu_{0} I_{i} I_{j} \frac{R_{i}+R_{j}}{A}\left\{\Lambda(k)\left[\frac{R_{j}}{R_{i}+R_{j}}\right]+\frac{E(k)}{1-k^{2}}\left[\frac{k^{2}}{2}-\frac{R_{j}}{R_{i}+R_{j}}\right]\right\} \mathrm{e}_{\mathrm{R}} \\
& -\mu_{0} I_{i} I_{j} \frac{Z_{i}-Z_{j}}{4}\left\{\Lambda(k)+\frac{E(k)}{1-k^{2}}\left(\frac{k^{2}}{2}-1\right)\right\} \mathrm{e}_{\mathbf{Z}}
\end{aligned}
$$

\section{D.1.2 Self Force of a Filamentary Current}

The magnetic energy of a single filamentary coil interacting with its own magnetic field is expressed as

$$
U_{i \rightarrow i}=\frac{\mu_{0}}{2} I_{i}^{2} R_{i}\left[\ln \left(\frac{8 R_{i}}{\pi}\right)-2\right]
$$

The gradient of this expression can be computed directly and is given by

$$
\nabla_{i} U_{i \rightarrow i}=\mathbf{e}_{\mathbf{R}} \frac{\mu_{0}}{2} I_{i}^{2}\left[\ln \left(\frac{8 R_{i}}{a}\right)-1\right]
$$

Computing the sclf force of this coil results in

$$
\mathrm{F}_{\mathrm{i} \rightarrow \mathrm{i}}=\frac{\mu_{1}}{2} I_{i}^{2}\left[\ln \left(\frac{\& R_{i}}{a}\right) \cdots 1\right] \mathbf{e}_{\mathrm{R}}
$$




\section{D.2 Distributed Currents}

\section{D.2.1 Force Between Two Coils}

The interaction energy of a distributed current $J_{i}$ in the magnetic field produced by a current $J_{j}$ is given by

$$
\begin{aligned}
U_{j \rightarrow i} & =\frac{1}{2} \int_{V} \mathbf{J}_{\mathbf{i}}(\mathbf{r}) \cdot \mathbf{A}_{\mathbf{j}}(\mathbf{r}) d \tau \\
& =\frac{\mu_{0} J_{i} J_{j}}{4} \int_{Z_{1}-\frac{h_{1}}{2}}^{Z_{1}+\frac{h_{1}}{2}} \int_{R_{1}-\frac{w_{1}}{2}}^{R_{1}+\frac{w_{i}}{2}} \int_{Z_{j}-\frac{h_{1}}{2}}^{Z_{j}+\frac{h_{1}}{2}} \int_{R_{j}-\frac{w_{1}}{2}}^{R_{j}+\frac{w_{1}}{2}} r^{\prime}\left(r^{\prime}\left(r, z ; r^{\prime}, z^{\prime}\right) d r^{\prime} d z^{\prime} d r d z\right.
\end{aligned}
$$

It has been shown that [23]

$$
\frac{d}{d \alpha} \int_{u_{0}(\alpha)}^{u_{1}(\alpha)} f(x, \alpha) d x=f\left(u_{1}, \alpha\right) \frac{d u_{1}}{d \alpha}-f\left(u_{0}, \alpha\right) \frac{d u_{0}}{d \alpha}+\int_{u_{0}(\alpha)}^{u_{1}(\alpha)} f_{i \alpha}(x, \alpha) d x
$$

with the proviso that $u_{0}(\alpha)$ are $u_{1}(\alpha)$ differentiable and $f(x, \alpha)$, and $f_{c x}(x, \alpha)$ are continuous. Using this expression to compute $\mathrm{F}_{\mathrm{j} \rightarrow \mathrm{i}}$ results in

$$
\begin{aligned}
& \mathbf{F}_{\mathbf{j} \rightarrow \mathrm{i}}=\mathrm{e}_{\mathbf{R}} \frac{\mu_{0} J_{i} J_{j}}{2} \int_{Z_{1}-\frac{h_{i}}{2}}^{Z_{\mathrm{t}}+\frac{h_{1}}{2}} \int_{Z_{3}-\frac{h_{1}}{2}}^{Z_{1}+\frac{h_{J}}{2}} \int_{R_{j}-\frac{w_{1}}{2}}^{R_{1}+\frac{w_{1}}{2}}\left(R_{i}+\frac{w_{i}}{2}\right) r^{\prime}\left(i\left(R_{i}+\frac{w_{i}}{2}, z ; r^{\prime}, z^{\prime}\right) d r^{\prime} d z^{\prime} d z\right. \\
& -\mathbf{e}_{\mathrm{R}} \frac{\mu_{1} J_{i} J_{j}}{2} \int_{Z_{1}-\frac{h_{1}}{2}}^{Z_{1}+\frac{h_{1}}{2}} \int_{Z_{1}-\frac{h_{2}}{2}}^{Z_{1}+\frac{h_{2}}{2}} \int_{R_{1}-\frac{w_{2}}{2}}^{R_{1}+\frac{w_{2}}{2}}\left(R_{i}-\frac{w_{i}}{2}\right) r^{\prime}\left(C_{1}\left(R_{i}-\frac{w_{i}}{2}, z ; r^{\prime}, z^{\prime}\right) d r^{\prime} d z^{\prime} d z\right.
\end{aligned}
$$

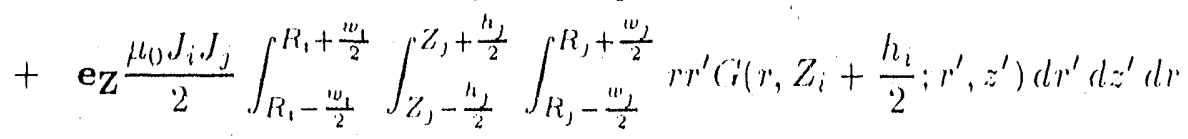

$$
\begin{aligned}
& \text { - } \mathrm{e}_{\mathrm{Z}} \frac{\mu_{0} J_{i} J_{j}}{2} \int_{R_{1}-\frac{m_{1}}{2}}^{R_{1}+\frac{m_{1}}{2}} \int_{Z_{3}-\frac{h_{j}}{2}}^{Z_{1}+\frac{h_{1}}{2}} \int_{R_{1}-\frac{u_{1}}{2}}^{R_{j}+\frac{m_{1}}{2}} r^{\prime} r^{\prime}\left(i\left(r, Z_{i}-\frac{h_{i}}{2} ; r^{\prime}, z^{\prime}\right) d r^{\prime} d z^{\prime} d r\right.
\end{aligned}
$$




\section{D.2.2 Self Force of a Distributed Current}

The energy of a single distributed current interacting with its own magnetic field is found from

$$
\begin{aligned}
U_{i \rightarrow i} & =\frac{1}{2} \int_{V} \mathbf{J}_{i}(\mathbf{r}) \cdot \mathbf{A}_{\mathbf{i}}(\mathbf{r}) d \tau \\
& =\frac{\mu_{0} J_{i}^{2}}{4} \int_{Z_{1}-\frac{h_{1}}{2}}^{Z_{1}+\frac{h_{1}}{2}} \int_{R_{1}-\frac{\omega_{1}}{2}}^{R_{1}+\frac{\omega_{1}}{2}} \int_{Z_{1}-\frac{h_{1}}{2}}^{Z_{1}+\frac{h_{1}}{2}} \int_{R_{1}-\frac{\omega_{1}}{2}}^{R_{1}+\frac{\omega_{1}}{2}} r^{\prime} G\left(r, z ; r^{\prime}, z^{\prime}\right) d r^{\prime} d z^{\prime} d r d z
\end{aligned}
$$

Using equation D.3 to compute the gradient of this expression gives:

$$
\begin{aligned}
\frac{\partial l_{i}-i}{\partial R_{i}} & =\frac{\mu_{0} J_{i}^{2}}{4} \int_{Z_{1}-\frac{w_{1}}{2}}^{Z_{1}+\frac{w_{1}}{2}} \int_{Z_{1}-\frac{h_{1}}{2}}^{Z_{i}+\frac{h_{1}}{2}} \int_{R_{1}-\frac{w_{1}}{2}}^{R_{1}+\frac{w_{1}}{2}}\left(R_{i}+\frac{w_{i}}{2}\right) r^{\prime}\left(r_{i}+\frac{w_{i}}{2}, z ; r^{\prime}, z^{\prime}\right) d r^{\prime} d z^{\prime} d z \\
& -\frac{\mu_{0} J_{i}^{2}}{4} \int_{Z_{1}-\frac{w_{i}}{2}}^{Z_{1}+\frac{w_{1}}{2}} \int_{Z_{1}-\frac{h_{1}}{2}}^{Z_{1}+\frac{h_{1}}{2}} \int_{R_{1}-\frac{w_{1}}{2}}^{R_{1}+\frac{w_{1}}{2}}\left(R_{i}-\frac{w_{i}}{2}\right) r^{\prime} C^{\prime}\left(R_{i}-\frac{w_{i}}{2}, z ; r^{\prime}, z^{\prime}\right) d r^{\prime} d z^{\prime} d z \\
& +\frac{\mu_{0} \cdot J_{i}^{2}}{4} \int_{Z_{1}-\frac{w_{1}}{2}}^{Z_{1}+\frac{w_{1}}{2}} \int_{R_{1}-\frac{w_{1}}{2}}^{R_{1}+\frac{w_{1}}{2}} \int_{Z_{1}-\frac{h_{1}}{2}}^{Z_{1}+\frac{h_{1}}{2}} r\left(R_{i}+\frac{w_{i}}{2}\right) C^{\prime}\left(r, z ; R_{i}+\frac{w_{i}}{2}, z^{\prime}\right) d z^{\prime} d r d z \\
& -\frac{\mu_{0} J_{i}^{2}}{4} \int_{Z_{1}-\frac{w_{1}}{2}}^{Z_{1}+\frac{w_{1}}{2}} \int_{R_{1}-\frac{w_{1}}{2}}^{R_{1}+\frac{w_{1}}{2}} \int_{Z_{1}-\frac{h_{1}}{2}}^{Z_{1}+\frac{h_{1}}{2}} r\left(R_{i}-\frac{w_{i}}{2}\right) C^{\prime}\left(r, z ; R_{i}-\frac{w_{i}}{2}, z^{\prime}\right) d z^{\prime} d r d z
\end{aligned}
$$

where conservation of flux has resulted in a sign reversal.

The final expression for the self-force is found by making use of the symmetry relations for (i developed in Appendix $\mathrm{C}$ and relabeling $r$ as $r^{\prime}$ in the last two integrals simplifies the preceeding expression to

$$
\begin{aligned}
\frac{\partial U_{i-i}}{\partial R_{i}} & =\frac{\mu_{0} J_{i}^{2}}{2} \int_{Z_{1}-\frac{m_{1}}{2}}^{Z_{1}+\frac{\omega_{1}}{2}} \int_{Z_{1}-\frac{h_{1}}{2}}^{Z_{1}+\frac{h_{1}}{2}} \int_{1 r}^{\beta} \beta r^{\prime}\left(r\left(\beta, z ; r^{\prime}, z^{\prime}\right) d r^{\prime} d z^{\prime} d z\right. \\
& -\frac{\mu_{0} J_{i}^{2}}{2} \int_{Z_{1}-\frac{\omega_{1}}{2}}^{Z_{1}+\frac{\omega_{1}}{2}} \int_{Z_{1}-\frac{h_{1}}{2}}^{Z_{2}+\frac{h_{1}}{2}} \int_{1 r}^{\beta} \alpha r^{\prime}\left(r\left(\alpha, z ; r^{\prime}, z^{\prime}\right) d r^{\prime} d z^{\prime} d z\right.
\end{aligned}
$$

where $\alpha$ and $\beta$ are constants given by:

$$
\begin{aligned}
& \alpha=R_{i}-\frac{w_{i}}{2} \\
& \beta=R_{i}+\frac{w_{i}}{2}
\end{aligned}
$$




\section{Appendix E}

\section{Gaussian Quadrature}

In this appendix, the Gaussian integration algorithm is reviewed. The algebraic transformations required to evaluate the various integrals presented in Appendix D are described and, as an example, Equation D.5 is expressed in terms of those transformations.

Most numerical integration (quadrature) schemes require that the values of the integrand be known at evenly spaced points (c.g. the Newton-Cotes formulac). This works well for cases where the integrand is known or even where the exact functional form of the integrand is unknown and only a set of discrete values are available for integration. For cases where the functional form of the integrand is known, however, it is possible to determine the points where the integrand is to be evaluated such that the accuracy of the scheme is increased. The purpose of Gaussian quadrature is to find an optimal manner for determining these points.

Gaussian quadrature relies on orthogonal functions to determine the optimal points. Of the schemes available, the one chosen here uses Legendre polynomials, which are orthogonal in the region $1-1,11$. This gives

$$
\int_{-1}^{1} P(x) d x=\sum_{i=1}^{n} c_{i} P\left(x_{i}\right)
$$

where the $x_{i}$ are the roots of the Legendre polynomial of degree 1 and the "i are nomalization coefficients. The values of $x_{i}$ and ${ } i$ for many values of $n$ are tabulated in 241 . This scheme is exact for all polynomials of degree at most $2 n-1$ and has an error of

$$
l_{t}=\frac{2^{2 n+1}[(n) !]^{1}}{(2 n+1)[(2 n) !]^{3}} f^{(2 n)}(n)
$$

where $n$ is the number of points used for quadrature, $f^{(2 n)}(x)$ is the $2 n$th derivative of $f(x)$, and $x$ is in the region $1-1,11$. 
The integral in equation E. 1 has limits of $[-1,1]$. Any set of limits $[1, b \mid$ in $x$ can be mapped into $|-1,1|$ in $\alpha$ by the transformation

$$
\alpha=\frac{2}{b-a}\left[w-\frac{b+a}{2}\right]
$$

so that

$$
\int_{a}^{b} f(x) d x=\frac{b-a}{2} \int_{-1}^{1} f\left(\frac{b+a}{2}+\frac{b-a}{2} \alpha\right) d x
$$

Throughout this thesis, the current density across a coil has been assumed constant:

$$
J_{i}=\frac{I_{i}}{w_{i} h_{i}}
$$

Combining this with the previous results allows us to write equation D.5 as

$$
\begin{aligned}
\mathbf{F} & =\mathbf{e}_{\rho} \frac{\mu_{0} I_{i}^{2}}{2} \frac{1}{8 w_{i}} \int_{-1}^{1} \int_{-1}^{1} \int_{-1}^{1}\left(R_{i}+\frac{w_{i}}{2}\right) g(\beta)\left(i\left(R_{i}+\frac{\omega_{i}}{2}, f(\alpha) ; g(\beta), h(\gamma)\right) d \beta d \gamma d l \alpha\right. \\
& -\mathbf{e}_{\rho} \frac{\mu_{0} I_{i}^{2}}{2} \frac{1}{8 w_{i}} \int_{-1}^{1} \int_{-1}^{1} \int_{-1}^{1}\left(R_{i}-\frac{w_{i}}{2}\right) g(\beta)\left(i\left(R_{i}-\frac{\omega_{i}}{2}, f(\alpha) ; g(\beta), h(\gamma)\right) d \phi d \gamma d l \alpha\right.
\end{aligned}
$$

where

$$
\begin{aligned}
& f(\alpha)=Z_{i}+\frac{h_{i}}{2} \alpha \\
& g(\beta)=R_{i}+\frac{w_{i}}{2} \beta \\
& h(\gamma)=Z_{i}+\frac{h_{i}}{2} \gamma
\end{aligned}
$$

The rapid convergence of this quadrature scheme is clearly illustrated in Figure E-1, where the coefficient of the self-force $\left(\frac{F_{1}-1}{\mu_{0} l_{1}^{2}}\right)$ of a coil with $R_{t}=0.50, Z_{i c}=0.0(0, w=0.05$ and $h=0.05 .5$ is plotted as a function of the number of quadrature points (n) used. The rapid convergence is especially interesting since the number of computations required to compute the force scales as $n^{3}$. For example, the self-force coefficient computed with 8 points is 2.0958 .11 , while that for 100 points is 2.092711 . The result of the 8 point scheme is only 0.15 f. 2 r cent greater than the result of the 1.00 point scheme, and requires approximately $5 \cdot 10^{-4}$ as much time to compute. Because of the reasonable accuracy and speed of the $x$ point scheme, it is used throughout the program to compute the required triple integrals. 


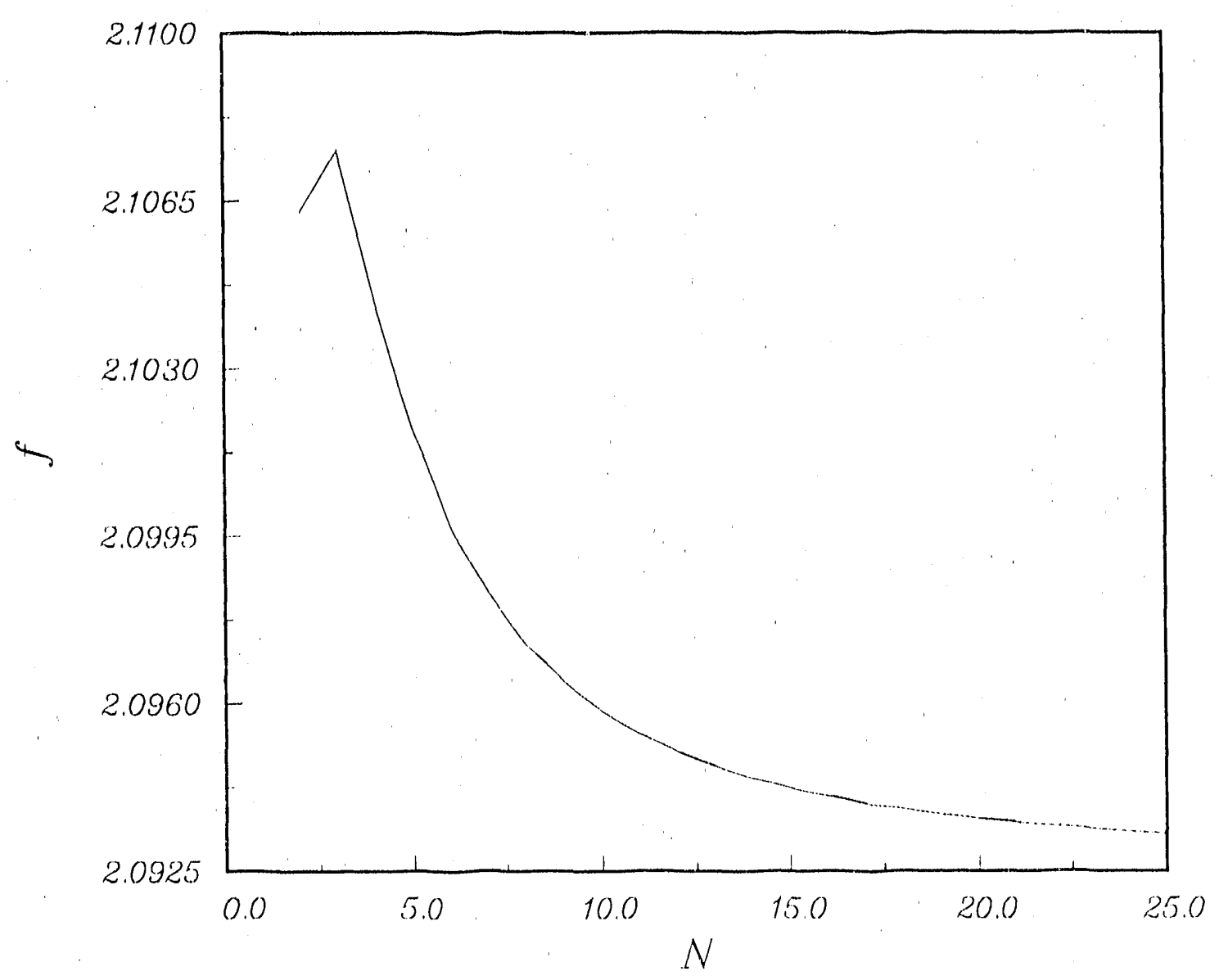

Figure E-1: Self-fore coefficient as a function of the number of cuadrature points used. 


\section{Bibliography}

(1) T. Baumeister, E. A. Avallone, and T. Baumeister III, editors. Mark's' Standard Handbook for Mechanical Engineers, pages 5-2 -5-51. McGraw-Hill Book Company, eighth edition, 1978.

[2] Richard L. Burden, J. Douglas Faires, and Albert C. Reynolds. Numericral Analysis, pages 448-453. PWS Publishers, second edition, 1981.

[3] J. P. Freidberg. Ideal Magnetohydrodynamics, pages 59-61. Plenum Press, 1987.

14] J. P. Freidberg. Ideal Mragnetohydrodynamins, pages 86-87. Plenum Press, 1987. Problem 4.5 .

[5] J. P. Freidberg and W. Grossman. Magnetohydrodynamic stability of a sharp boundary model of tokamak. Physics of Fluids, 18(11):1494, 1975.

[6] J. P. Freidberg, W. Grossman, and F. A. Haas. Stability of a high-ib,l $l=3$ stcllerator. Physics of Fluids, 19(10):1599, 1976.

[7] J. P. Freidberg and F. A. Haas. Kink instabilitics in a high-is tokamak with elliptic cross section. Physics of Filuids, 17(2):440, 1974.

181 P. E. Gill, W. Murray, arid M. H. Wright. Pratical Optimization, pages 167--182. Academic Press, 1981.

19) P. E. Gill, W. Murray, and M. H. Wright. Prartical ()ptimization, pages 1-5. Academic Press, 1981.

110] I. S. Gradshteyn and I. M. Ryzhik. Table of Lintgruls, Se riess, and Prodlurls, page 907. Academic Press, fourth edition, 1980. 
[11] S. P. Hakkarainen and J. P. Freidberg. Reconstruction of Vacuum Flux Surfaces from Diagnostic Measurements in a Tokamak. Technical Report, Massachusetts Institute of Technology, 1987. M.I.T. Report PFC/RR-87-2.

[12] Scott W. Haney. Methods for the Design and Optimization of Shaped Tokamaks. PhD thesis, Massachusetts Institute of Technology, May 1988.

[13] Scott W. Haney. Methods for the Design and Optimization of Shaped Tokamaks. PhD thesis, Massachusetts Institute of Technology, May 1988. pages $105-141$.

[14] Scott W. Haney. 'Methods for the Design and Optimization of Shaped Tokamaks. PhD thesis, Massachusetts Institute of Technology, May 1988. pages $128-132$.

[15] Scott W. Haney, Methods for the Design and Optimization of Shaped Tokamaks. PhD thesis, Massachusetts Institute of Technology, May 1988. pages 93 - 104.

[16] F. B. Hildebrand. Advanced Calculus for Applications, pages 357-359. Prentice-Hall, Inc., second edition, 1976.

[17] F. B. Hildebrand. Advanced C'alculus for Applications, pages 352-354. Prentice-Hall, Inc., second edition, 1976.

[18] F. B. Hildebrand. Advanced Calculus for Applications, pages 215--223. Prentice-Hall, inc., second edition, 1976.

[19] J. P. Holman. Heai İransfer, pages 35 - 37. McGraw-Hill Book Company, fifth edition, 1981.

[20] J. Mathews and R. L. Walker. Mathematical Methods of Physics, page 260. Benjamin/Cummings Publishing Company, second edition, 1970.

121] P. B. Powell, editor. Planning for Softuare Validation, Verification, and Testing. U.S. Dept. of Commerce, National Bureau of Standards, 1982. NBS Special Publication; 500-98.

[22] W. H. Press, B. P. Flannery, S. A. Teuolsky, and W. T. Vettering. Numerical Recipes in C, page 29. Cambridge University Press, 1988.

123] I.S. Sokolnikoff and R. M. Redheffer. Mathematics of Physices and Modern Enginerering, pages 347-350. McGraw-Hill Book Company, second edition, 1966. 
[24] A. H. Stroud and D. Secrest. Ciaussian Quadrature Formulas. Prentice-Hall, Inc., 1966.

[25] R. K. Wangsness. Electromagnetic Fields, pages 323-326. John Wiley \& Sons, 1979. 


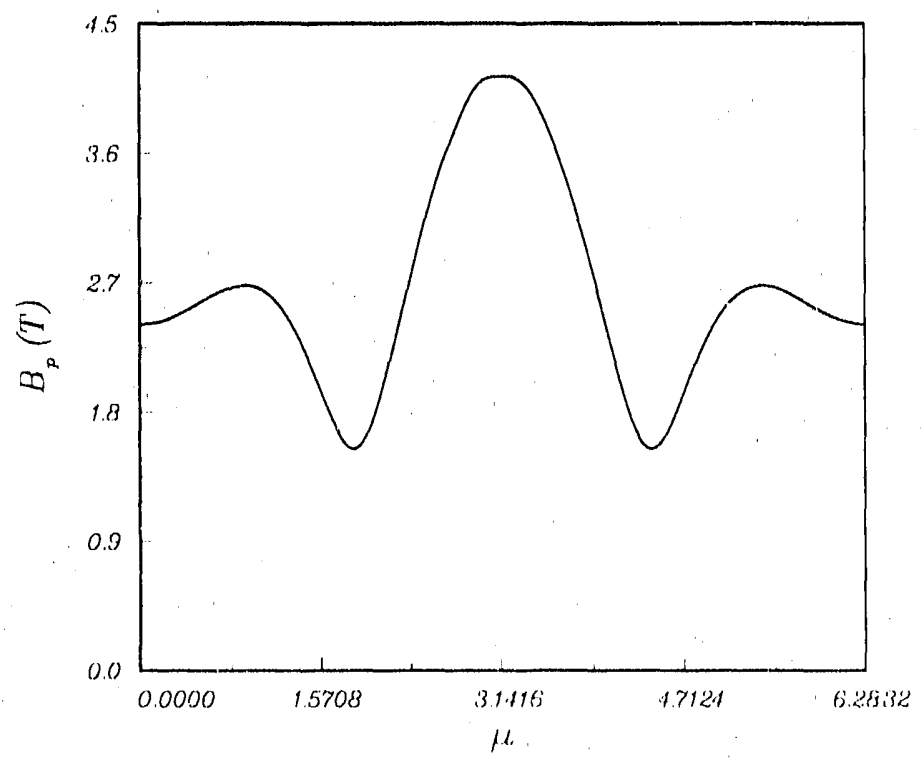

Figure 3-8: Poloidal fields for the symmetrie tese case. The solid line is the polotedal field ant the plasma surface for the equilibrium. The dotted line (indistinguishable heres) represents the combined fields of the plasma and the poloidal fiedd coils.

listed in Table 3.6 is computed from

$$
d=(i-1) / i
$$

where $d$ is the difference, $i$ is the input current and $o$ is the output current. A negative difference simply means that the coil current found by CPFC was larger than the input current.

Inspection of the values in Table 3.6 shows that the CPFC program reproduces the input coil currents to an accuracy of better than $0.02 \%$. This indicates that the program is correctly calculating the coil currents for this case. Further indication of how well the coil currents are being reproduced is illustrated in Figure 3-8, which compares the input poloidal field with that generated by the coil currents listed in Table 3.6. The field error $(1)$ for this case is $1.1 \cdot 10^{-1} \%$.

For the asymmetric case, two of the filaments listed in Table 3.4 were removed and the current carried by those filaments was redistributed accordingly. The resulting asymmetric plasma current distribution is modeled by the filaments in Table 3.7. The poloidal field coil currents and positions used for this case are identical to those listed in 3.5. Figure 3-9 shows the coils, plasma filaments, 

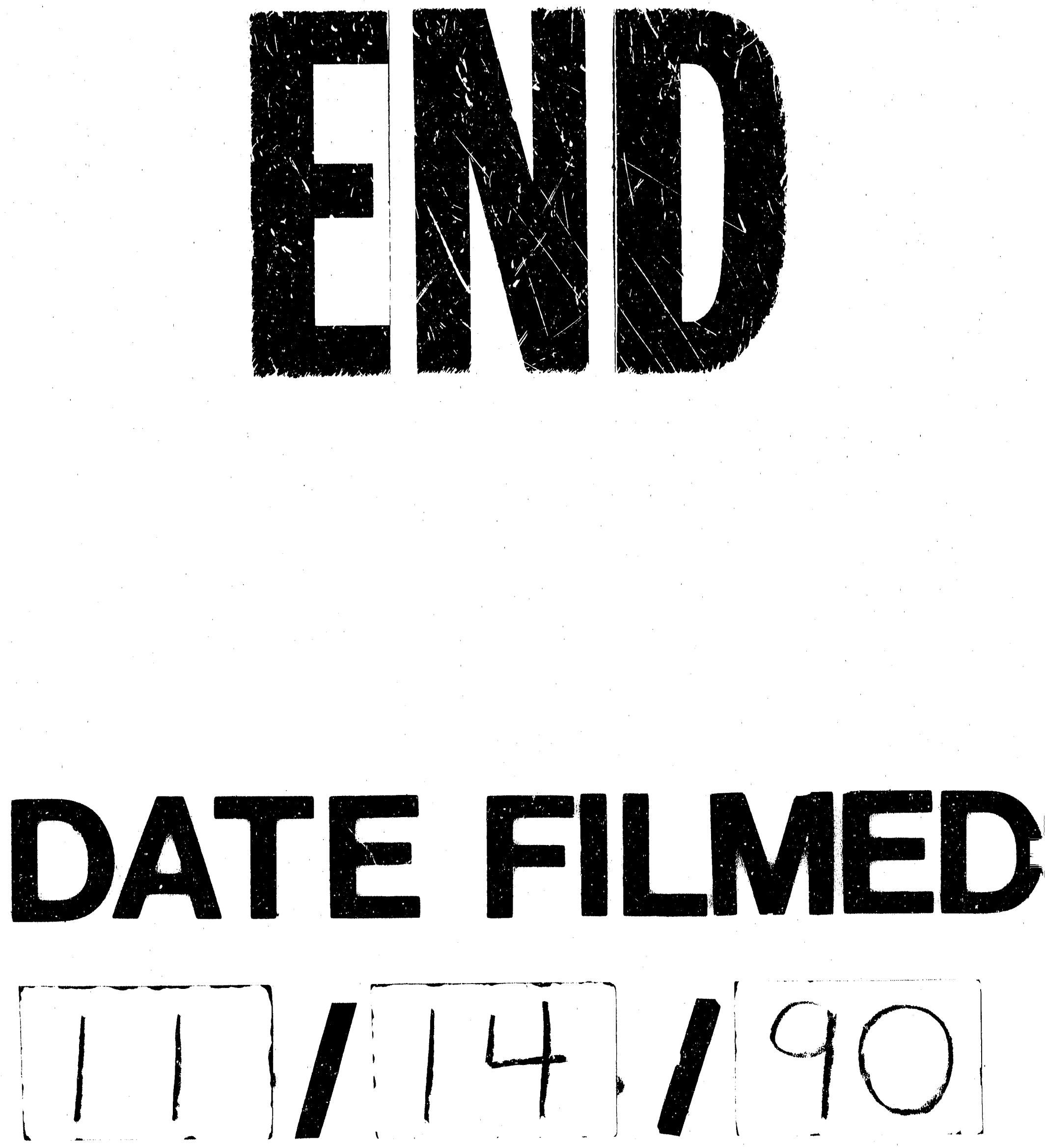
